\title{
Poxviruses capture host genes by LINE-1 retrotransposition
}

\author{
Sarah M. Fixsen ${ }^{1}$, Kelsey R. Cone ${ }^{1}$, Stephen A. Goldstein ${ }^{1}$, Thomas A. Sasani ${ }^{1}$, Aaron R. \\ Quinlan$^{1}$, Stefan Rothenburg ${ }^{2}, \&$ Nels C. Elde ${ }^{1 *}$ \\ ${ }^{1}$ Department of Human Genetics, University of Utah, Salt Lake City, USA \\ ${ }^{2}$ Department of Microbiology, University of California, Davis, USA
}

*Correspondence to: nelde@genetics.utah.edu

\begin{abstract}
Horizontal gene transfer (HGT) provides a major source of genetic variation. Many viruses, including poxviruses, encode genes with crucial functions directly gained by gene transfer from hosts. The mechanism of transfer to poxvirus genomes is unknown. Using genome analysis and experimental screens of infected cells, we discovered a central role for Long Interspersed Nuclear Element-1 (LINE-1) retrotransposition in HGT to virus genomes. The process recapitulates processed pseudogene generation, but with host messenger RNA directed into virus genomes. Intriguingly, hallmark features of retrotransposition appear to favor virus adaption through rapid duplication of captured host genes on arrival. Our study reveals a previously unrecognized conduit of genetic traffic with fundamental implications for the evolution of many virus classes and their hosts.
\end{abstract}

\section{Summary}

Active selfish genetic elements in infected cells aid virus adaptation by catalyzing transfer of host genes to virus genomes. 


\section{Introduction}

Vertical inheritance of genetic material from parent to offspring can be augmented by horizontal gene transfer (HGT) (Keeling and Palmer, 2008). HGT can shortcut gradual mutational processes by facilitating adaptive leaps through single mutational events where genes can be transferred across kingdoms of life (Keeling and Palmer, 2008). HGT plays a central role in the diversification of many infectious microbes. This process is particularly well-described in bacteria, where HGT contributes to the emerging crisis of multi-antibiotic resistant pathogens (Wijayawardena et al., 2013). Many classes of viruses also acquire host genes by HGT (Caprari et al., 2015). A conspicuous example is Rous Sarcoma virus, in which recognition of the horizontal transfer of the host c-Src gene into the virus genome led to the discovery of oncogenes (Swanstrom et al., 1983). It has become increasingly clear that acquisition of host genes is a common mechanism by which viruses of many classes gain adaptive advantages to propagate and evolve. Despite the prevalence of HGT in diverse viruses and its importance in virus evolution, the mechanisms by which genes are transferred from host to virus genomes are not understood.

To investigate mechanisms of transfer we examined poxviruses, which encode a plethora of host genes gained by HGT. Based on phylogenetic analysis, more than $25 \%$ of poxvirus genes were acquired by HGT from hosts (Hughes and Friedman, 2005), although similarities not evident in sequence comparisons but revealed by shared protein structures suggest that a higher proportion of genes were acquired by horizontal transfer (Bahar et al., 2011). Many captured genes adapt to act as inhibitors of host immune defenses to favor virus replication (Elde and Malik, 2009).

Some act as host range factors because their deletion leads to restriction of infectible cell types or species, implying that new acquisitions may aid in host jumps (Bratke et al., 2013; Haller et al., 2014). While the most notorious poxvirus, variola virus which caused smallpox, has been eradicated, other extant poxvirus strains are capable of infecting humans and pose the risk of new pandemics (Sklenovska and Van Ranst, 2018). 


\section{Results}

We hypothesized that examination of genes recently acquired by HGT might provide clues for revealing mechanisms of gene transfer. Virus homologs of the host Golgi Anti-Apoptotic Protein (GAAP; also called transmembrane Bax inhibitor motif protein family 4 or TMBIM4), found in several orthopoxviruses, are $\sim 75 \%$ identical to human GAAP (Gubser et al., 2007; Saraiva et al., 2013), suggesting a recent acquisition and/or highly conserved function favoring virus replication. Strikingly, we observed nearly identical 21 base pair sequences flanking the open reading frame of virus GAAP (VGAAP), indicative of a target site duplication (TSD), consistent with gene capture resulting from LINE-1 mediated retrotransposition of host mRNA (Figs. 1A and S1). The vGAAP gene is situated near one of two identical inverted terminal repeat (ITR) regions of the virus genome, and on opposite ends of the genome in various viruses, suggesting that it was originally transferred into the ITR. A single copy of the putative $21 \mathrm{bp}$ TSD at an analogous position near the other ITR in the cowpox genome suggests a pre-integration sequence or pseudo empty site (Fig. 1A). Together with a published account of a non-autonomous Short Interspersed Nuclear Element (SINE) in the genome of taterapox virus (Piskurek and Okada, 2007), these observations suggest that host genes can be retrotransposed into poxvirus genomes by LINE-1 activity in a process akin to the generation of processed pseudogenes (Esnault et al., 2000).

Phylogenetic analysis revealed a single origin for the horizontal transfer of GAAP into poxvirus genomes. In addition, at least two other classes of DNA viruses, herpesviruses and iridoviruses, independently acquired GAAP by HGT (Fig. 1B) (Bellehumeur et al., 2016; de Groof et al., 2015). Another study revealed that the S1 gene of Squirrel Monkey cytomegalovirus encodes a protein more than $95 \%$ identical to squirrel monkey Signaling lymphocytic activation molecule family 6 (SLAMF6), followed by portions of the 3' untranslated region (UTR) of the host mRNA (Perez-Carmona et al., 2015). These findings suggest that many DNA viruses acquire host genes by retrotransposition. However, the majority of host to virus gene transfers lack features of retrotransposition, which can quickly degrade through mutation, motivating our development of an experimental strategy for capturing HGT events in real time. 


\section{FIGURE 1}

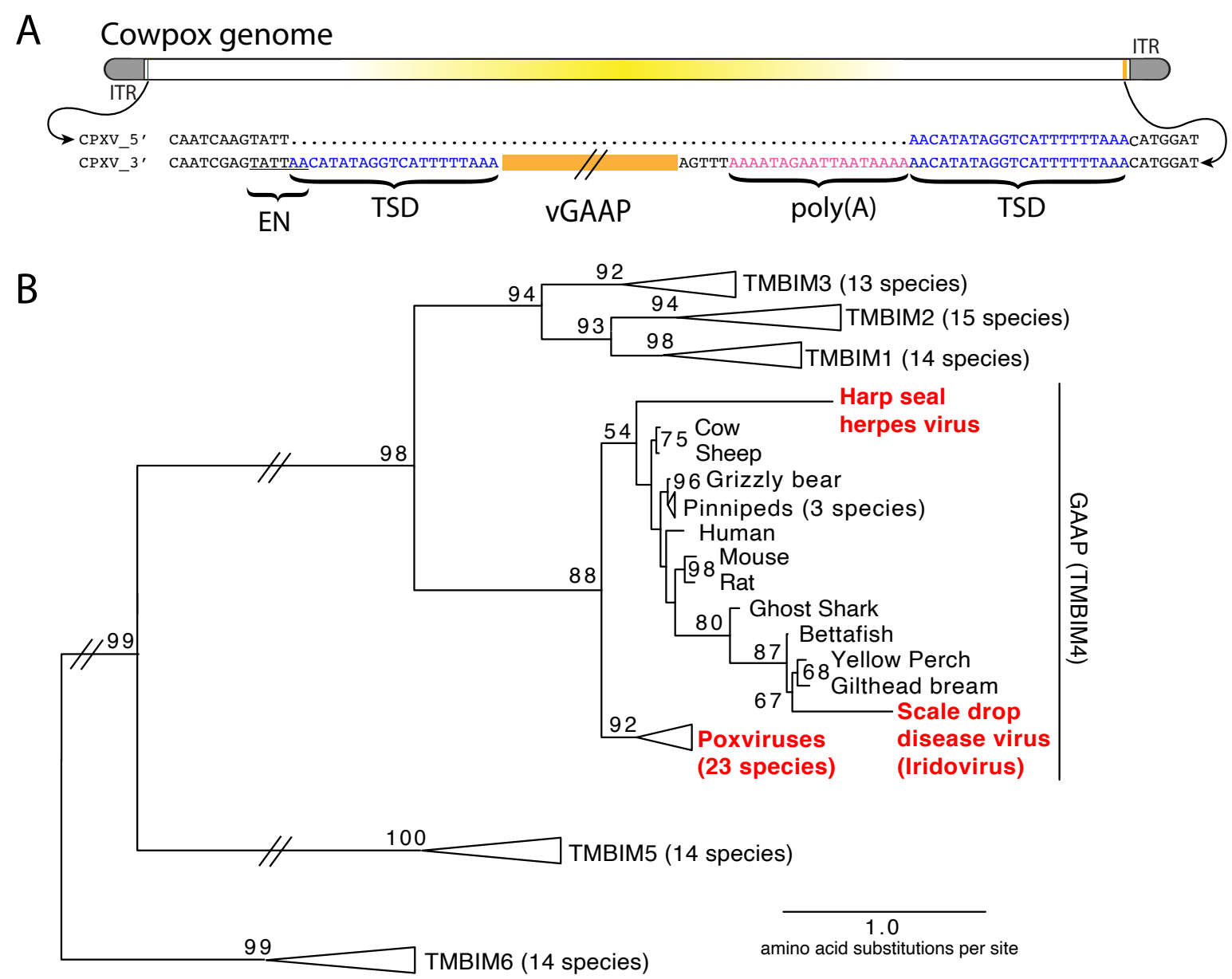

Fig. 1. Retrotransposon-mediated transfer of GAAP into poxvirus genomes. (A) The poxvirus gene vGAAP (orange) is encoded near the inverted terminal repeat (ITR; gray) of cowpox genomes. Signatures of retrotransposition include TSDs (blue), a LINE-1-like endonuclease site (EN; underlined), and a partially degraded poly(A) tail (pink). Some cowpox genomes (CPXV_5'; top line) contain a pseudo-empty site, with a single copy of the TSD sequence. (B) Phylogeny of TMBIM proteins and virus-encoded TMBIM4s (red). Bootstrap values $>50$ are indicated.

We developed a selection scheme using vaccinia virus (Copenhagen strain), which encodes nearly 200 genes in a 191,737 bp genome (See methods). We focused on K3L, a gene that was presumably acquired from a host by an ancient poxvirus based on sequence identity with human eukaryotic initiation factor $2 \alpha$ (eIF2 $\alpha ; 27.6 \%$ identical) (Beattie et al., 1991; Elde et al., 2009; Elde and Malik, 2009). K3L blocks the antiviral Protein Kinase R (PKR) pathway from arresting protein translation during viral infections by phosphorylating eIF2 $\alpha$ (Fig. S2) (Beattie et al., 1991). Using a mutant virus deleted for K3L and another PKR inhibitor, E3L (Brennan et al., 
2014), we infected a rabbit kidney (RK13) cell line expressing complementing chromosomeintegrated copies of $\mathrm{K} 3 \mathrm{~L}$ fused to the gene encoding mCherry fluorescent protein (RK13-K3L) (Fig. 2A and see Methods for details). Viruses grown in RK13-K3L cells were transferred to a non-complementing baby hamster kidney (BHK) cell line to select for viruses that "reclaimed" the mCherry-K3L gene and/or somehow adapted in the absence of K3L to block host cell PKR and replicate.

We screened $\sim 500$ million viruses over many experiments and recovered ten virus isolates from mCherry positive infected cell clones. In the restrictive BHK cells, viruses encoding $\mathrm{K} 3 \mathrm{~L}$ replicate 100-fold better than the parent virus deleted for K3L (Fig. 2B), providing strong selective pressure to screen for adapted viruses. After plaque purification of mCherry isolates, we infected BHK cells with each recovered virus isolate and observed titers similar to viruses encoding K3L (Fig. 2C). By sequencing the genome of each recovered virus isolate, we found that the mCherry-K3L fusion gene was integrated into the virus genome at a unique position in every isolate (Figs. 2D and S3), providing the opportunity to compare each independent integration event.

All integrations were consistent with a mechanism of LINE-1 retrotransposition-mediated HGT. The sequence of each transferred gene revealed hallmark features of mRNA gene capture, including 5' and 3' UTRs, precise intron splicing, and poly(A) tails (Fig. 3). For seven of ten isolates, TSDs flanked mCherry-K3L insertions and contained cut site sequences matching the consensus (TTTT/AA) of LINE-1 endonuclease (Fig. 3C). Although there were no TSDs flanking mCherry-K3L in isolates 5, 9, and 10, these integrations might result from a endonuclease-independent LINE-1 mechanism at DNA breaks (Morrish et al., 2002). Because our experiments lack any exogenous reverse transcriptase, we tested for endogenous activity in the RK13 cells and detected robust LINE-1 activity (Fig. S4). These results suggest that LINE-1 mediated retrotransposition may be a common mechanism of HGT into poxvirus genomes. 
FIGURE 2

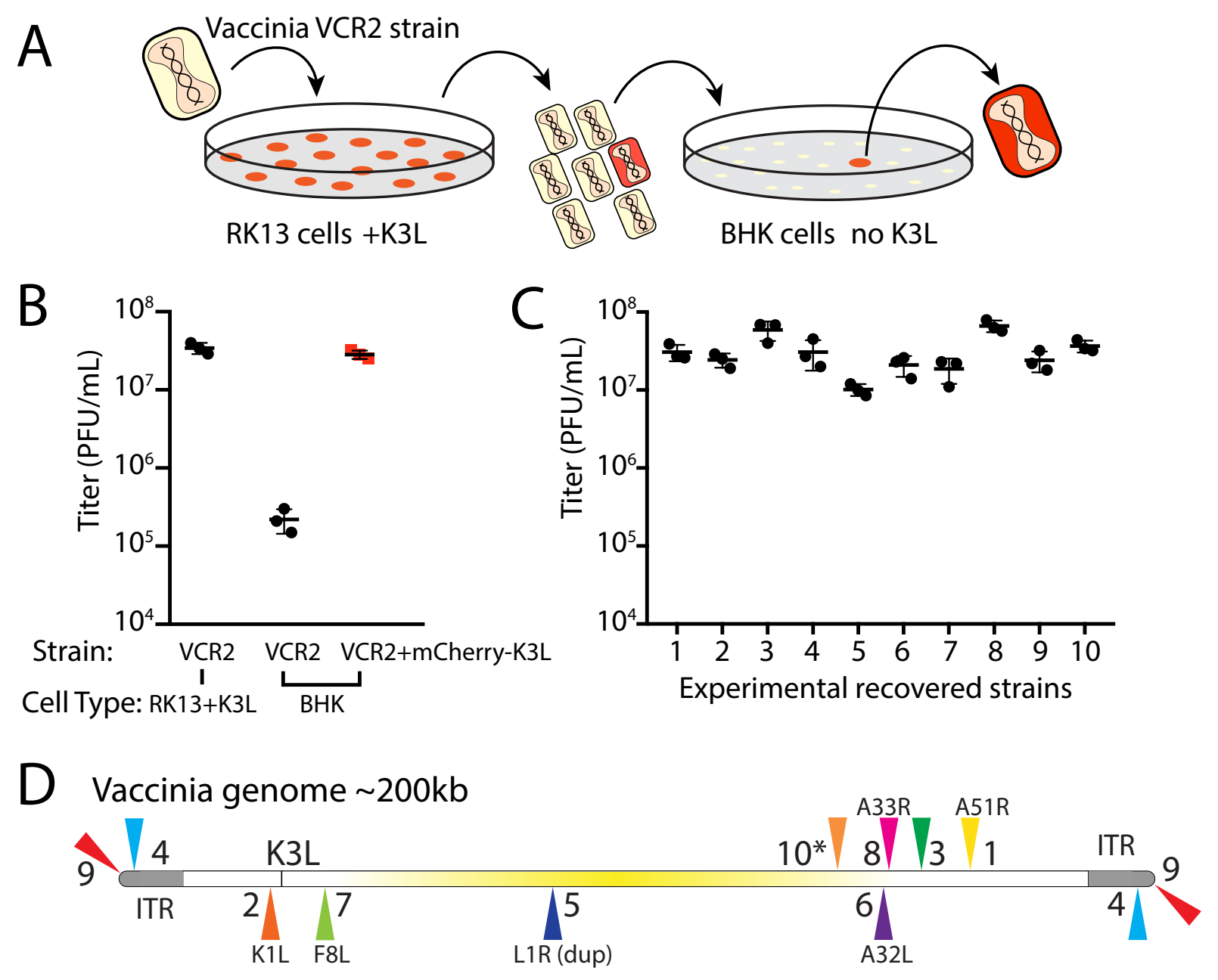

Fig. 2. Experimental capture of K3L by horizontal gene transfer. (A) Viruses lacking K3L (VCR2) replicated in RK13 cells expressing mCherry-K3L and were transferred to BHK cells lacking K3L to select for capture of mCherry-K3L. (B) Replication of VCR2 in RK13-K3L cells and BHK cells compared to VCR2+mCherry-K3L (see Methods). (C) Replication of recovered isolates in BHK cells. Three biological replicates of each strain/isolate are shown in (B) and (C). (D) Vaccinia genome illustrating K3L integrations in recovered isolates indicated by colored triangles. Endogenous K3L location is shown. The central region of the genome is highlighted in yellow. Triangles above and below the genome denote positive and negative sense orientation respectively. Virus genes interrupted by K3L integrations are indicated. The asterisk denotes a genomic rearrangement in isolate 10 (Fig S3).

Poxvirus genomes are arranged with the essential genes, shared by all poxviruses, near the center of the genome. Clade- or species-specific genes, which more commonly exhibit evidence of HGT, are enriched near the ends of the genome (Lefkowitz et al., 2006; McLysaght et al., 2003). This pattern may reflect that gene transfer into the central region of genome is more likely to 
interrupt genes vital for virus replication (Lefkowitz et al., 2006). Consistent with this, all but one recovered isolate integrated $\mathrm{K} 3 \mathrm{~L}$ outside a roughly defined central region of the genome containing 90 core chordopoxvirus genes (Lefkowitz et al., 2006) (Fig. 2C). In the case of isolate 5, the K3L integration involved a duplication of two essential genes, G9R and L1R (Fig. S3). Because gene duplications can appear frequently in poxvirus genomes (Hughes and Friedman, 2005), this activity might enable the persistence of horizontally transferred genes by preserving existing ones, consistent with a process described in a contemporaneous study (Rahman et. al., 2020).

FIGURE 3

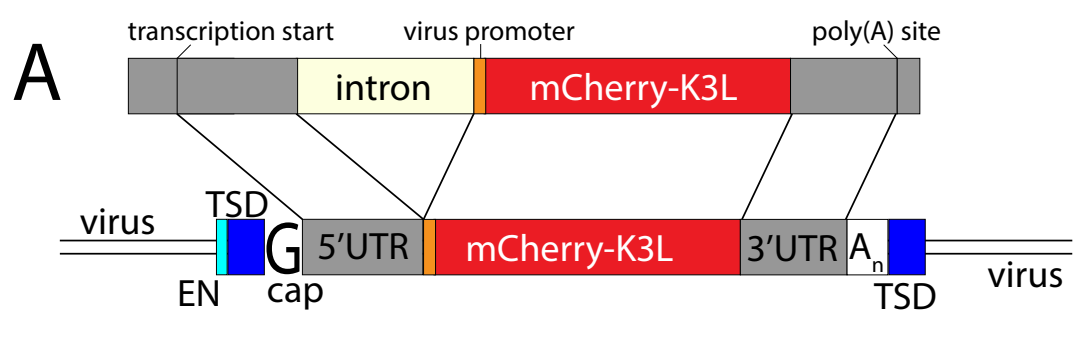

\begin{tabular}{|c|c|c|c|c|c|c|c|c|}
\hline B Strain & EN site & TSD length & $\mathrm{G} \mathrm{cap}$ & $5^{\prime} \mathrm{UTR}$ & spliced & $3^{\prime} \mathrm{UTR}$ & poly(A) & dsDNA break \\
\hline 1 & TCTT/AA & $17 \mathrm{bp}$ & $\mathrm{Y}$ & $\mathrm{Y}$ & $\mathrm{Y}$ & $\mathrm{Y}$ & $\mathrm{Y}$ & None \\
\hline 2 & TTCT/AA & $16 \mathrm{bp}$ & $\mathrm{Y}$ & $\mathrm{Y}$ & $\mathrm{Y}$ & $\mathrm{Y}$ & $\mathrm{Y}$ & None \\
\hline 3 & $\mathrm{TTCT} / \mathrm{AA}$ & $19 \mathrm{bp}$ & $\mathrm{Y}$ & $\mathrm{Y}$ & $\mathrm{Y}$ & $\mathrm{Y}$ & $\mathrm{Y}$ & None \\
\hline 4 & $\mathrm{TTTT} / \mathrm{AT}$ & $14 \mathrm{bp}$ & $\mathrm{N}$ & $\mathrm{Y}$ & $\mathrm{Y}$ & $\mathrm{Y}$ & $\mathrm{Y}$ & None \\
\hline 5 & None & None & $\mathrm{Y}$ & $\mathrm{Y}$ & $\mathrm{Y}$ & $\mathrm{Y}$ & $\mathrm{Y}$ & tandem dup \\
\hline 6 & $\mathrm{TTCT} / \mathrm{AA}$ & $15 \mathrm{bp}$ & $\mathrm{N}$ & $\mathrm{Y}$ & $\mathrm{Y}$ & $\mathrm{Y}$ & $\mathrm{Y}$ & None \\
\hline 7 & $\mathrm{TCTT} / \mathrm{AA}$ & $18 \mathrm{bp}$ & $\mathrm{Y}$ & $\mathrm{Y}$ & $\mathrm{Y}$ & $\mathrm{Y}$ & $\mathrm{Y}$ & None \\
\hline 8 & $\mathrm{TTCT} / \mathrm{AT}$ & $16 \mathrm{bp}$ & $\mathrm{N}$ & $\mathrm{Y}$ & $\mathrm{Y}$ & $\mathrm{Y}$ & $\mathrm{Y}$ & None \\
\hline 9 & $\mathrm{ND}$ & $\mathrm{ND}$ & $\mathrm{N}$ & $\mathrm{Y}$ & $\mathrm{Y}$ & $\mathrm{Y}$ & $\mathrm{Y}$ & concatemer \\
\hline 10 & None & None & $\mathrm{Y}$ & $\mathrm{Y}$ & $\mathrm{Y}$ & $\mathrm{Y}$ & $\mathrm{Y}$ & translocation \\
\hline
\end{tabular}

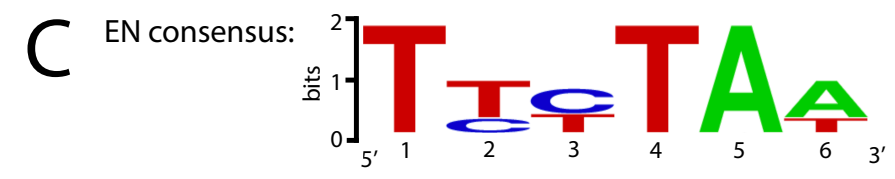

Fig. 3. All virus isolates exhibit signatures of LINE-1 mediated retrotransposition. (A) Schematic of construct integrated in RK13-K3L cells (top) compared to K3L integrations in recovered viruses (bottom). Shared features include spliced introns, 5' and 3' untranslated regions (UTRs), and poly(A) tails $\left(\mathrm{A}_{n}\right)$. Seven isolates have target site duplications (TSDs, blue) with LINE-1 endonuclease cut sites (EN, cyan). Six isolates encode a guanine (G cap) adjacent to the 5'UTR, indicative of 7-methylguanylate mRNA capping. (B) Table highlights details of each isolate. (C) Logos plot of endonuclease cut site consensus sequence.

Previous studies found that duplication of poxvirus genes, followed by rapid gene copy amplification, can boost virus replication (Brennan et al., 2014; Elde et al., 2012). In our 
analysis, we discovered two isolates where K3L had undergone polymorphic copy number increases (isolates 4 and 5; Fig. 4A). Homologous recombination, including unequal crossovers leading to duplications, requires as little as 16bp of homology in poxviruses (Yao and Evans, 2001), suggesting that LINE-1 induced TSDs might facilitate gene amplification. Genes transferred to within the ITR are duplicated on the other end by replication-based mechanisms (McFadden and Dales, 1979), as we observed in isolates 4 and 9 (Fig. 2C). Genes integrated into the near the termini of the genome by HGT, like isolate 4, may be especially prone to duplication due to stretches of short, high copy tandem repeats found in and near the inverted terminal repeat regions (Fig. 4A). HGT therefore likely occurs evenly across poxvirus genomes, but with an enrichment of transferred genes persisting near the ends, where insults to essential genes are less probable and duplication-based adaptation is favored (Fig. S5). Consistent with this idea, gene families found only in specific lineages of poxviruses, indicating more recent acquisition, are more likely to be present in multiple copies than more host genes acquired in (Hughes and Friedman, 2005).

While our experimental system revealed LINE-1 driven mechanisms of HGT, the scheme involved selection for viruses regaining an existing virus gene. To better model virus adaptation following acquisition of a host gene, we engineered viruses with the human eIF2 $\alpha$ gene in place of K3L (Fig. S6; see Methods). We performed four serial infections of viruses expressing eIF2 $\alpha$ or an eIF2 $\alpha$ variant lacking the PKR phosphorylation site (S51A) in RK13 cells. While the recombinant viruses replicate weakly, we observed a nearly 10 -fold increase in virus replication for both eIF2 $\alpha$-encoding viruses after passaging (Fig. 4B). By sequencing the eIF2 $\alpha$ region in evolved virus strains, we found independent gene copy increases of eIF2 $\alpha$ in both viruses (Fig. 4C). These results support a critical role for gene copy increases following HGT in poxvirus evolution, as also observed for K3L and other genes (Brennan et al., 2014; Elde et al., 2012).

\section{Discussion}

Horizontal gene transfer is an important process facilitating virus evolution (Caprari et al., 2015; Deeg et al., 2018; Hughes and Friedman, 2005; Koonin and Yutin, 2019; McLysaght et al., 2003). Our discovery of LINE-1 retrotransposition-mediated HGT from host to poxvirus 
genomes has several notable implications. In addition to self-propagation in "host" genomes, retrotransposition to virus genomes could greatly extend the range of LINE-1 or related elements that subsequently mobilize to a newly infected virus host (Piskurek and Okada, 2007). The extensive host range of some poxviruses, for example between rodent and primate species, suggests the possibility of cross host lineage transfer of LINE-1s, non-autonomous Alus, and host transcripts between mammals. A process involving the frequent appearance of DNA transposons in some insect viruses suggests a parallel mechanism for the spread of transposable elements between species (Gilbert et al., 2016). In cases of retrotransposition of host transcripts, the resulting processed pseudogene products are enriched for highly expressed genes, including ribosomal genes (Zhang et al., 2004). The over representation of certain transcripts might provide clues for understanding and predicting how viruses might adapt with repurposed genes mimicking host functions (Elde and Malik, 2009). Currently recognized cases of HGT involving oncogenes and regulators of translation, like eIF $2 \alpha$, provide cases of transcripts exhibiting high gene expression and cellular functions easily repurposed to promote virus replication.

Repetitive target site duplications generated by LINE-1 activity may also enhance poxvirus adaptation by facilitating rapid gene copy number amplification of horizontally transferred genes on arrival. Increases in gene copy number augment the probability of beneficial mutations appearing, allowing for swift diversification of newly acquired genes (Elde et al., 2012). Thus, form follows function as the repetitive termini of poxvirus genomes further enable the emergence and persistence of acquired genes predominately at the ends of the genome (Fig. 4D). As beneficial virus gene variants endure, they end up closer to the center of the genome as subsequently captured genes appear near the ends.

Similar mechanisms of virus adaptation involving HGT may be shared by other diverse classes of nucleocytoplasmic DNA viruses (NCLDVs), in addition to poxviruses (Deeg et al., 2018; Koonin and Yutin, 2019). Many giant viruses, which can exceed a megabase in genome size and infect diverse host species, encode a variety of genes acquired by HGT, including ribosomal genes. Given a primary role for LINE-1 elements in facilitating gene transfer, we speculate that other classes of transposable elements may spur NCDLV evolution by mobilizing host genes into virus genomes across diverse ecosystems. 
FIGURE 4
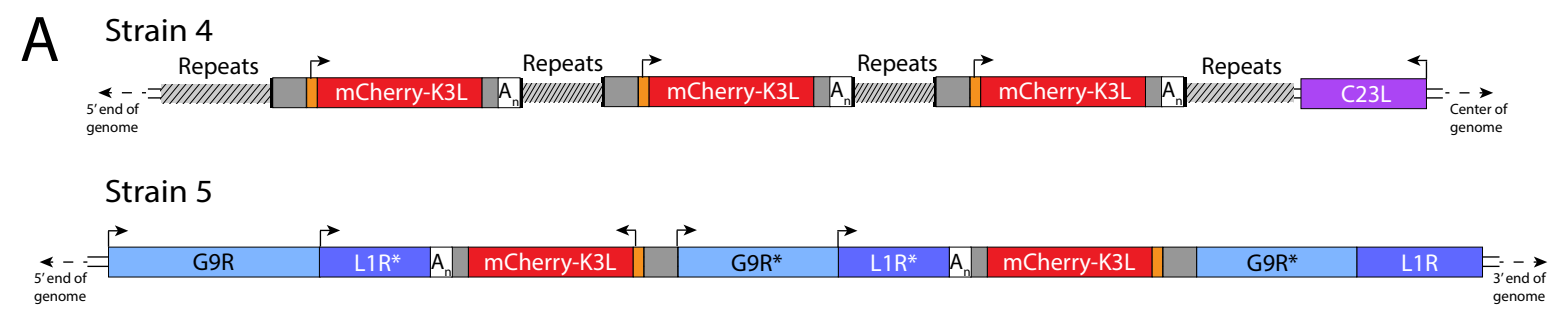

B

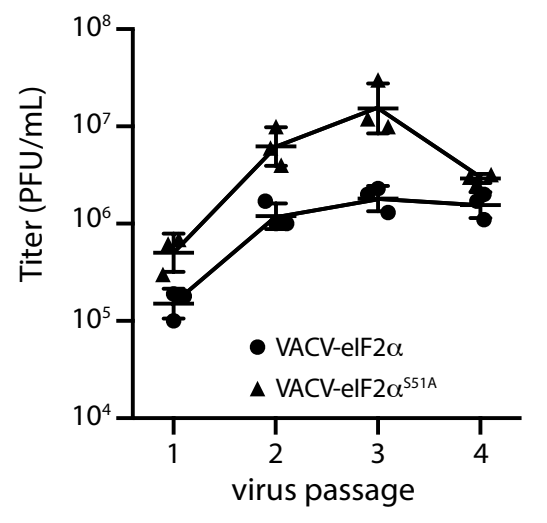

C elF2a-GFP duplication
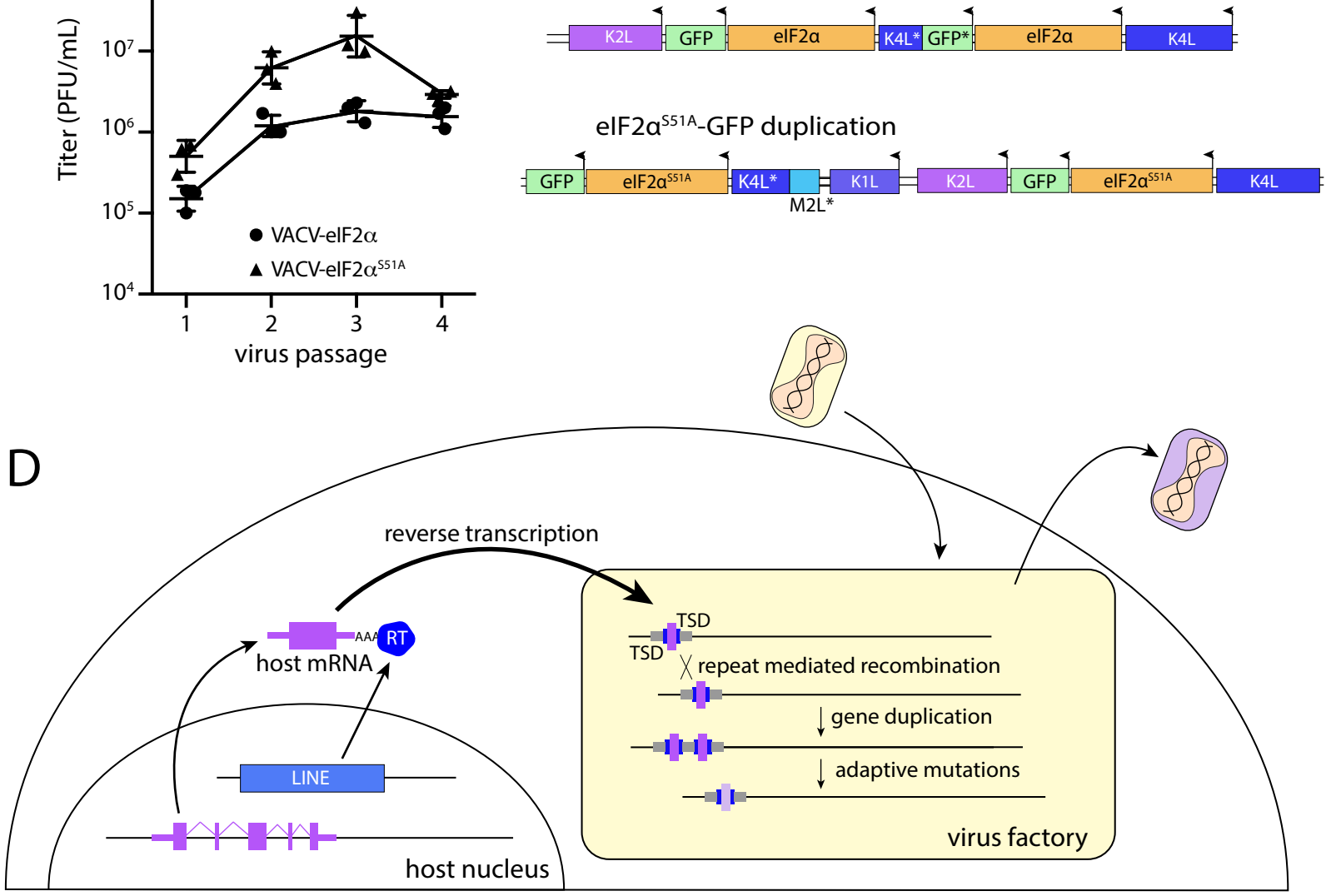

Fig. 4: Homology driven gene duplication of K3L and eIF2 $\alpha$. (A) Schematics of mCherry-K3L duplications in isolates 4 and 5. (B) Virus titers after serial infections of eIF2 $\alpha$ viruses in HeLa cells (see Methods). (C) Schematics of eIF2 $\alpha$ duplications following virus passaging. Asterisks denote gene truncations (D) Model diagram highlighting how host genes (purple) are retrotransposed by LINE-1 reverse transcriptase (RT, blue), into virus genomes (yellow box). Following horizontal transfer, gene duplidation is facilitated by unequal crossover recombination between TSDs (blue) or flanking repeat sequence (gray). 


\section{Materials and methods}

Phylogenetic analysis and sequence comparison of vGAAP

TMBIM sequences for various species were downloaded from NCBI (Supplemental data) and aligned using the COBALT Multiple Alignment Tool. Amino acid Phylip alignments (see supplemental materials) were analyzed by PhyML (http://www.atgc-montpellier.fr/phyml/) for tree building with 100 bootstraps (Guindon et al., 2010).

\section{Cell culture}

BHK and HeLa cells were cultured in Dulbecco minimum essential medium (DMEM; HyClone) supplemented with 10\% FBS, $2 \mathrm{mM}$ L-glutamine and $100 \mu \mathrm{g} / \mathrm{mL}$ of pen/strep. RK13 cells were cultured in MEM-alpha supplemented with 10\% fetal bovine serum (FBS; Gibco), 2 mM Lglutamine (HyClone) and $100 \mu \mathrm{g} / \mathrm{mL}$ of penicillin-streptomycin (pen/strep; HyClone).

RK13 cells expressing SLP-mCherry-K3L (RK13-K3L) were generated by transfecting 3x10 5 RK13-E3K3 cells (Brennan et al., 2014) with 2.5ug piggybac vector (see supplemental sequences) and $0.5 \mathrm{ug}$ transposase vector (gift from Ed Grow, University of Utah) with 9uL Fugene-HD. Cells with the piggybac construct integrated were selected by the presence of 1-2 $\mathrm{ug} / \mathrm{mL}$ puromycin, and populations were monitored for mCherry expression. After two weeks of selection, remaining polyclonal cells were propagated in MEM-alpha media supplemented with 10\% FBS, $2 \mathrm{mM}$ L-glutamine and $100 \mu \mathrm{g} / \mathrm{mL}$ of pen/strep. All cultures were maintained and infections performed at $37^{\circ} \mathrm{C}$ in a humidified $5 \% \mathrm{CO}_{2}$ incubator.

\section{$\underline{\text { Virus strains }}$}

VC-2 refers to the Vaccinia virus-Copenhagen strain (Goebel et al., 1990) and VCR2 is a genetically modified isolate of VC-2 deleted for E3L and K3L (Brennan et al., 2014). To generate VCR2+mCherry-K3L, we cloned pBlue_165_mCherry-K3L (see supplemental sequences) into the MCS of pBluescriptIIKS (-) (Addgene). This plasmid was transfected into BHK cells, which were then infected with VCR2 virus. Recombinant viruses were plaque purified in BHK cells and checked for purity by PCR amplifying across the mCherry-K3L insertion site. The amplicon was gel purified and sequenced. 
To make eIF2 $\alpha$ viruses, sequences flanking K3L from VACV were amplified from VC-2 viral DNA: $680 \mathrm{bp}$ of 5' homologous sequence was amplified with the primers K2Lflank_F (5'CTTCTTATC GATTTTTTATACCGAACATAAAAATAAGGTTAATTA) and K2Lflank_R (5'-CTTCTTCATATGG TGATTGTATTTCCTTGCAATTTAG), and 1024bp of 3' homologous sequence (including the native K3L promoter) was amplified with the primers K4Lflank_F (5'-CGTCGTGCGGCCGCCTTGTTAACGGGCTCGTAAATT) and K4Lflank_R (5'-CGA GCGGAGCTCGTACGATACATAGATATTACAAATATCCTAG). A VACV synthetic early/late promoter (SLP)(Chakrabarti et al., 1997) was created by annealing primers SLP_F (5' TCGACAATTGGATCAGCTTTTTTTTTTTTTTTTTTGGCATATAAATA AGAAGCTTCCCGGGTCTAGAC) and SLP_R (5'-AGCTCAGATCTGGGCCCTTCG AAGAATAAATATACGGTTTTTTTTTTTTTTTTTTCGACTAGGTTAAC). EGFP was amplified from pN1-EGFP (Addgene) using primers EGFP_F (5'GGAGGACTCGAGATGGTGAGCAAGGGCGA) and EGFP_R (5'-GGAGGTATCGA TTTACTTGTACAGCTCGTCCATGC). Each PCR product was digested with restriction enzymes (New England Biolabs), gel purified (Zymo Research), and sequentially cloned into pB.2 as follows: 5' flank with ClaI and NdeI, 3' flank with NotI and SacI, SLP with SalI and XhoI, and EGFP with XhoI and ClaI. The resulting plasmid contained EGFP following SLP, between the two K3L flanking sequences (pB.2-EGFP). The K3L open reading frame was amplified from VC-2 viral DNA using primers K3L_F (5'-GTTGTAG GATCCATGCTTGCATTTTGTTATTCGTTGC) and K3L_R (5'-GTTCTTGTCGACTT ATTGATGTCTACACATCCTTTTG). The eIF2 $\alpha$ gene was amplified from human cDNA using primers eIF2 $\alpha$ F (5'-GATGTAGGATCCATGCCGGGTCTAAGTTGTAGAT) and eIF2 $\alpha \_R$ (5'-CTACTTGTCGACTTAATCTTCAGCTTTGGCTTCCAT). The resulting PCR products were cloned into pB.2-EGFP with BamHI and SalI, placing K3L or eIF2 $\alpha$ immediately following the native K3L promoter, and upstream of SLP-EGFP to create pB.2-K3L and pB.2eIF2 $\alpha$, respectively. Site-directed mutagenesis was performed on pB.2-eIF $2 \alpha$ or pB.2-eIF2 $\alpha-\Delta C$ using primers eIF2 $\alpha \_S 51$ A_F (5'-GATAC GCCTTCTGGCTAATTCACTAAGAAGAATCATGCCTTC) and eIF2 $\alpha$ S51A_R (5'GAAGGCATGATTCTTCTTAGTGAATTAGCCAGAAGGCGTATC) to generate pB.2-eIF2 $\alpha$ S51A. 
Recombinant eIF2 $\alpha$-encoding viruses were constructed by replacing the K3L gene using homologous recombination. RK13-E3K3 cells were infected with VCR2 (MOI = 1.0) and transfected at 1-hour post-infection with pB.2-EGFP, pB.2-K3L, pB.2-eIF2 $\alpha$, or pB.2-eIF2 $\alpha$ S51A plasmids by use of FuGENE6 (Promega) according to the manufacturer's protocol. Infected cells were collected at 48 hours post infection, and viruses were released by one freezethaw cycle followed by sonication. Resulting viruses were plaque purified in RK13-E3K3 cells four times, selecting for recombinants expressing EGFP. Final virus clones were verified by PCR and sequencing of viral DNA across the K2L-K4L region of the genome.

Experimental screen for horizontal gene transfer events

Confluent 150mm dishes of RK13-K3L cells were infected with VCR2 at a MOI of 0.1. Cellassociated virus was collected after 48 hours of infection, and released from the cell by one freeze-thaw cycle followed by sonication as described (Isaacs, 2004). Confluent 150mm dishes of BHK cells were then infected with $\sim 10^{6}$ PFUs of this virus stock for 7 days, during which they were monitored for mCherry expression. 500 plates were screened, which accounts for an estimated 500 million viruses. For mCherry positive clones observed by fluorescence microscopy, cell-associated virus was collected and released by one freeze-thaw cycle followed by sonication. mCherry-expressing virus was then plaque purified in BHK cells as described (Isaacs, 2004).

\section{Analysis of horizontal gene transfer events by inverse PCR (iPCR)}

Virus DNA was extracted from mCherry-expressing clones as previously described from infected BHK cells (Esposito et al., 1981). Purified DNA was digested with XbaI and SpeI or BgIII (New England Biolabs (NEB)), diluted, and ligated (Quick ligase; NEB) to circularize linear fragments. Primers pointing away from each other in both mCherry (F-

CGTGGAACAGTACGAACGCG and R- CCATGTTATCCTCCTCGCCC) and K3L (FGAGCATAATCCTTCTCGTATACTC and R- GAATATAGGGATAAACTGGTAGGG) were used to amplify regions flanking mCherry-K3L insertions. Resulting PCR bands were gel purified and Sanger sequenced with the iPCR primers. From this sequencing, the general location of the mCherry-K3L insert could be inferred. Primers flanking this region were then used to amplify the putative insertion, along with flanking sequences. These amplicons were then 
gel purified, Sanger sequenced, and compared to the parent (VCR2) genome to characterize gene integrations. Because isolates 7 and 9 incorporated mCherry-K3L within the repetitive ITR, each end was PCR amplified separately using unique flanking primers and internal primers (mCherry$\mathrm{R}$ and K3L-F, above). However, only the 5' end of the insertion of isolate 9 was amplified, despite multiple attempts. Thus, we cannot be sure whether this isolate includes a TSD or was cut at a LINE-1 endonuclease site.

\section{Long read genome sequencing of virus isolates}

Virus particles from plaques expressing mCherry-K3L were isolated from BHK cells and virus cores were purified by ultracentrifugation through a $36 \%$ sucrose cushion at 60k rcf for $1 \mathrm{hr}$. Virus DNA was extracted as previously described (Esposito et al., 1981). The SQK-LSK108 library kit (Oxford Nanopore Technologies) was used to prep isolate 1 DNA, which was sequenced on a FLO-MIN106 cell. A SQK-RBK001 kit was used to prep DNA from isolates 25, which were multiplexed on a FLO-MIN107 cell. Isolates 6-10 were also multiplexed using the SQK-RBK004 library prep kit, and sequenced on FLO-MIN107. Reads were base-called with the Oxford Nanopore Albacore program and aligned to a reference genome that included the VCR2 genome on one contig and the cellularly-expressed mCherry-K3L construct on a separate contig using default NGMLR parameters (Sedlazeck et al., 2018). Integrative Genomics Viewer (IGV) software (Broad Institute) was used to visualize insertions (Robinson et al., 2011).

Sequencing data is deposited in the NCBI SRA database, accession number: PRJNA614958.

\section{Measuring titers of virus strains and isolates}

We plated $5 \times 10^{6}$ cells in $100 \mathrm{~mm}$ dishes and infected them 16 hours later at an MOI of 0.1 . Three plates (biological replicates) were infected with each isolate or strain. After 48 hours, media was aspirated, and cell-associated virus stock was collected by one freeze-thaw and sonication cycle. Then, 6-well plates were seeded with $5 \times 10^{5}$ BHK cells per well, and, 16 hours later, infected with 10 -fold serial dilutions of virus stock ( 2 wells per dilution) in $200 \mathrm{uL}$ media. After 2 hours at $37^{\circ} \mathrm{C}, 2 \mathrm{~mL}$ of media was added to each well. Cells were fixed and stained with $20 \%$ Methanol + $0.2 \%$ crystal violet 48 hours post infection. Wells with 10-100 plaques were counted and averaged to calculate virus titer. Each of the biological replicates is shown in Figure 2 and Figure 4 along with an average of the three and calculated standard deviations. 


\section{$\underline{\text { Alu assay of RK13 cells }}$}

$5 \times 10^{5}$ HA-HeLa or RK13 cells were plated per 100mm dish ( $3 \mathrm{x}$ each cell type). 16 hours later, each plate was transfected with 5ug Alu-Neo (Dewannieux et al., 2003). After 2 days, all cells were treated with $2 \mathrm{ug} / \mathrm{mL}$ G418. Media was replaced daily for 7 days, after which time it was removed, and cells were fixed and stained with $20 \%$ Methanol $+0.2 \%$ crystal violet.

\section{Detection of duplication events}

Single Nanopore reads with multiple tandem copies of the mCherry-K3L fusion gene were analyzed using IGV (Robinson et al., 2011). Duplications were confirmed by PCR, using the inverse PCR primers in mCherry (F- CGTGGAACAGTACGAACGCG and RCCATGTTATCCTCCTCGCCC). PCR amplicons were then gel purified and Sanger sequenced to determine break points. However, due to the repetitive nature of the sequence flanking the isolate 7 insertion, the exact breakpoint was not able to be determined.

Serial passage of VACV-eIF $2 \alpha$ virus strains

For each passage, 150-mm dishes were seeded with an aliquot from the same stock RK13- cells

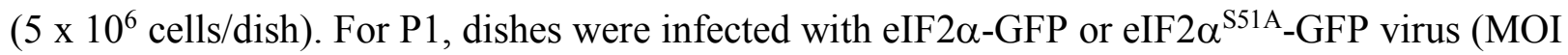
$=1.0$ ) for 2 hours in $5 \mathrm{~mL}$ and then supplemented with $15 \mathrm{~mL}$ medium. After 48 hours, cells were washed, pelleted, and resuspended in $1 \mathrm{~mL}$ of medium. Virus was released by one freeze-thaw cycle followed by sonication. $900 \mu 1$ of virus was then used to infect a new dish of cells for P2, and the process was repeated for subsequent passages. Viral titers were determined using the remaining $100 \mu 1$ of reserved virus stocks from each passage by 48-hour plaque assay in RK13E3K3 cells.

\section{Analysis of viral-encoded eIF2 $\alpha$ genes}

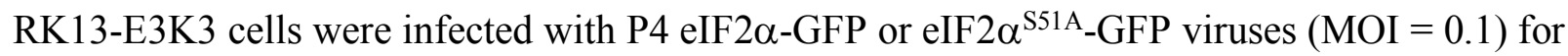
24 hours. Virus-infected cells were collected, and total viral DNA extracted as previously described(Esposito et al., 1981). The region between K2L and K4L containing the different viralencoded eIF2 $\alpha$ genes was amplified by PCR with primers K2L_seq_F and K4L_seq_R (5'GGCATTGGTAAATCCTTGCAGA and 5'-CACCTTTTAGTAGGACTAGTATCGTACAA, respectively). SNV detection was performed by sequencing across eIF2 $\alpha$ using primers eIF $2 \alpha \_F$ 
and eIF2 $\alpha \_$R for eIF2 $\alpha-$ GFP and eIF $2 \alpha^{\text {S51A }}$-GFP PCR products, CNV analysis was performed by PCR using primers eIF2 $\alpha$ rep_F (5'-CCTCCTATGGAAGCCAAAGCTGAAGATGAA) and eIF2 $\alpha$ rep_R (5'-CCTCCTATCTACAACTTAGACC CGGCAT) for eIF2 $\alpha$-GFP and

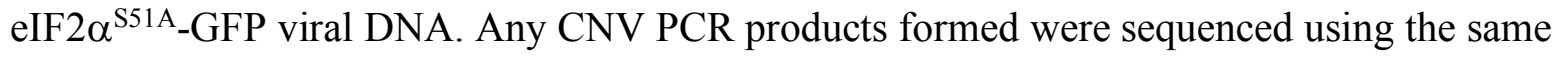
primers for breakpoint detection.

\section{References}

Bahar, M.W., Graham, S.C., Chen, R.A., Cooray, S., Smith, G.L., Stuart, D.I., and Grimes, J.M. (2011). How vaccinia virus has evolved to subvert the host immune response. J Struct Biol 175, 127-134.

Beattie, E., Tartaglia, J., and Paoletti, E. (1991). Vaccinia virus-encoded eIF-2 alpha homolog abrogates the antiviral effect of interferon. Virology 183, 419-422.

Bellehumeur, C., Nielsen, O., Measures, L., Harwood, L., Goldstein, T., Boyle, B., and Gagnon, C.A. (2016). Herpesviruses Including Novel Gammaherpesviruses Are Widespread among Phocid Seal Species in Canada. J Wildl Dis 52, 70-81.

Bratke, K.A., McLysaght, A., and Rothenburg, S. (2013). A survey of host range genes in poxvirus genomes. Infect Genet Evol 14, 406-425.

Brennan, G., Kitzman, J.O., Rothenburg, S., Shendure, J., and Geballe, A.P. (2014). Adaptive gene amplification as an intermediate step in the expansion of virus host range. PLoS Pathog 10, e1004002.

Caprari, S., Metzler, S., Lengauer, T., and Kalinina, O.V. (2015). Sequence and Structure Analysis of Distantly-Related Viruses Reveals Extensive Gene Transfer between Viruses and Hosts and among Viruses. Viruses 7, 5388-5409.

Chakrabarti, S., Sisler, J., and Moss, B. (1997). Compact, synthetic, vaccinia virus early/late promoter for protein expression. Biotechniques 23, 1094-1097.

de Groof, A., Guelen, L., Deijs, M., van der Wal, Y., Miyata, M., Ng, K.S., van Grinsven, L., Simmelink, B., Biermann, Y., Grisez, L., et al. (2015). A Novel Virus Causes Scale Drop Disease in Lates calcarifer. PLoS Pathog 11, e1005074. 
Deeg, C.M., Chow, C.T., and Suttle, C.A. (2018). The kinetoplastid-infecting Bodo saltans virus $(\mathrm{BsV})$, a window into the most abundant giant viruses in the sea. Elife 7.

Dewannieux, M., Esnault, C., and Heidmann, T. (2003). LINE-mediated retrotransposition of marked Alu sequences. Nat Genet 35, 41-48.

Elde, N.C., Child, S.J., Eickbush, M.T., Kitzman, J.O., Rogers, K.S., Shendure, J., Geballe, A.P., and Malik, H.S. (2012). Poxviruses deploy genomic accordions to adapt rapidly against host antiviral defenses. Cell 150, 831-841.

Elde, N.C., Child, S.J., Geballe, A.P., and Malik, H.S. (2009). Protein kinase R reveals an evolutionary model for defeating viral mimicry. Nature 457, 485-489.

Elde, N.C., and Malik, H.S. (2009). The evolutionary conundrum of pathogen mimicry. Nat Rev Microbiol 7, 787-797.

Esnault, C., Maestre, J., and Heidmann, T. (2000). Human LINE retrotransposons generate processed pseudogenes. Nat Genet 24, 363-367.

Esposito, J., Condit, R., and Obijeski, J. (1981). The preparation of orthopoxvirus DNA. J Virol Methods 2, 175-179.

Gilbert, C., Peccoud, J., Chateigner, A., Moumen, B., Cordaux, R., and Herniou, E.A. (2016).

Continuous Influx of Genetic Material from Host to Virus Populations. PLoS Genet 12, e1005838.

Goebel, S.J., Johnson, G.P., Perkus, M.E., Davis, S.W., Winslow, J.P., and Paoletti, E. (1990).

The complete DNA sequence of vaccinia virus. Virology 179, 247-266, 517-263.

Gubser, C., Bergamaschi, D., Hollinshead, M., Lu, X., van Kuppeveld, F.J., and Smith, G.L. (2007). A new inhibitor of apoptosis from vaccinia virus and eukaryotes. PLoS Pathog 3, e17. Guindon, S., Dufayard, J.F., Lefort, V., Anisimova, M., Hordijk, W., and Gascuel, O. (2010). New Algorithms and Methods to Estimate Maximum-Likelihood Phylogenies: Assessing the Performance of PhyML 3.0. Systematic Biology 59, 307-321.

Haller, S.L., Peng, C., McFadden, G., and Rothenburg, S. (2014). Poxviruses and the evolution of host range and virulence. Infect Genet Evol 21, 15-40.

Hughes, A.L., and Friedman, R. (2005). Poxvirus genome evolution by gene gain and loss. Mol Phylogenet Evol 35, 186-195.

Isaacs, S.N., ed. (2004). Vaccinia Virus and Poxvirology (Humana Press). 
Keeling, P.J., and Palmer, J.D. (2008). Horizontal gene transfer in eukaryotic evolution. Nat Rev Genet 9, 605-618.

Koonin, E.V., and Yutin, N. (2019). Evolution of the Large Nucleocytoplasmic DNA Viruses of Eukaryotes and Convergent Origins of Viral Gigantism. Adv Virus Res 103, 167-202.

Lefkowitz, E.J., Wang, C., and Upton, C. (2006). Poxviruses: past, present and future. Virus Res 117, 105-118.

McFadden, G., and Dales, S. (1979). Biogenesis of poxviruses: mirror-image deletions in vaccinia virus DNA. Cell 18, 101-108.

McLysaght, A., Baldi, P.F., and Gaut, B.S. (2003). Extensive gene gain associated with adaptive evolution of poxviruses. Proc Natl Acad Sci U S A 100, 15655-15660.

Morrish, T.A., Gilbert, N., Myers, J.S., Vincent, B.J., Stamato, T.D., Taccioli, G.E., Batzer, M.A., and Moran, J.V. (2002). DNA repair mediated by endonuclease-independent LINE-1 retrotransposition. Nat Genet 31, 159-165.

Perez-Carmona, N., Farre, D., Martinez-Vicente, P., Terhorst, C., Engel, P., and Angulo, A. (2015). Signaling Lymphocytic Activation Molecule Family Receptor Homologs in New World Monkey Cytomegaloviruses. J Virol 89, 11323-11336.

Piskurek, O., and Okada, N. (2007). Poxviruses as possible vectors for horizontal transfer of retroposons from reptiles to mammals. Proc Natl Acad Sci U S A 104, 12046-12051.

Robinson, J.T., Thorvaldsdóttir, H., Winckler, W., Guttman, M., Lander, E.S., Getz, G., and Mesirov, J.P. (2011). Integrative genomics viewer. Nature Biotechnology 29, 24-26.

Saraiva, N., Prole, D.L., Carrara, G., Maluquer de Motes, C., Johnson, B.F., Byrne, B., Taylor, C.W., and Smith, G.L. (2013). Human and viral Golgi anti-apoptotic proteins (GAAPs) oligomerize via different mechanisms and monomeric GAAP inhibits apoptosis and modulates calcium. J Biol Chem 288, 13057-13067.

Sedlazeck, F.J., Rescheneder, P., Smolka, M., Fang, H., Nattestad, M., von Haeseler, A., and Schatz, M.C. (2018). Accurate detection of complex structural variations using single-molecule sequencing. Nat Methods 15, 461-468.

Sklenovska, N., and Van Ranst, M. (2018). Emergence of Monkeypox as the Most Important Orthopoxvirus Infection in Humans. Front Public Health 6, 241.

Swanstrom, R., Parker, R.C., Varmus, H.E., and Bishop, J.M. (1983). Transduction of a cellular oncogene: the genesis of Rous sarcoma virus. Proc Natl Acad Sci U S A 80, 2519-2523. 
Wijayawardena, B.K., Minchella, D.J., and DeWoody, J.A. (2013). Hosts, parasites, and horizontal gene transfer. Trends Parasitol 29, 329-338.

Yao, X.D., and Evans, D.H. (2001). Effects of DNA structure and homology length on vaccinia virus recombination. J Virol 75, 6923-6932.

Zhang, Z., Carriero, N., and Gerstein, M. (2004). Comparative analysis of processed pseudogenes in the mouse and human genomes. Trends Genet 20,62-67.

Acknowledgments We thank D. Hancks, E. Choung, and E. Grow for reagents and advice. Funding This work was supported by NIH grants R01GM114514 and R35GM134936 (N.C.E.), T32GM007464 (S.M.F. and T.A.S), and T32AI055434 (K.S.R.). N.C.E. is a Burroughs Wellcome Fund Investigator in the Pathogenesis of Infectious Disease and H.A. and Edna Benning Presidential Endowed Chair.

Author contributions N.C.E. and S.R. conceptualized the project. N.C.E., S.M.F. and S.R. contributed to experimental design. S.M.F. and K.R.C. performed experiments. S.M.F, K.R.C., and T.A.S. analyzed and validated data. S.M.F., S.A.G. and N.C.E. visualized data. N.C.E. supervised. S.M.F wrote the original draft and N.C.E., S.M.F., and S.A.G. reviewed and edited the manuscript.

Competing interests The authors declare no competing interests.

Data and materials availability Bioproject PRJNA614958 


\section{Supplemental Materials}

Figures S1-S6

Table S1-S2

Supplementary sequences

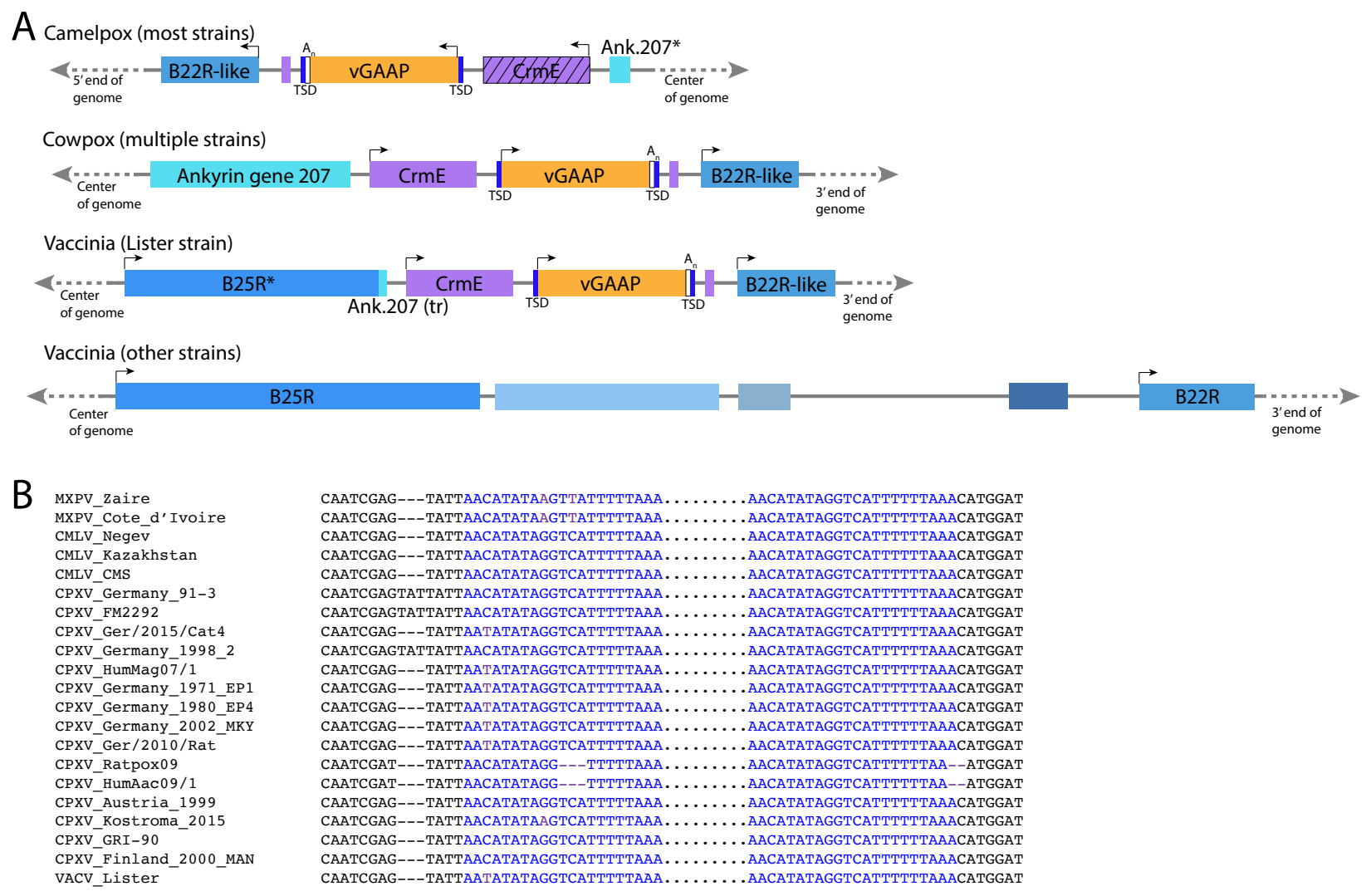

Fig. S1.

(A) v-GAAP (orange bar) is encoded by many orthopox species, including most camelpox strains, many cowpox strains, and at least one vaccinia strain. In camelpox viruses, V-GAAP is encoded on the left end of the genome; in all other viruses, v-GAAP is on the right end. In all viruses, v-GAAP is flanked by $\mathrm{CrmE}$ and vaccinia-B22R. In vaccinia strains that do not encode $\mathrm{V}$-GAAP, CrmE is also missing, suggesting recombination of the region between B22R and B25R, which is truncated in the Lister strain. (B) Alignment of target site duplications (blue) and flanking sequence in $21 \mathrm{v}$-GAAP-encoding poxvirus strains. MPXV, monkeypox; CMLV, camelpox; CPXV, cowpox; VACV, vaccinia. Asterisks denote gene truncations. 


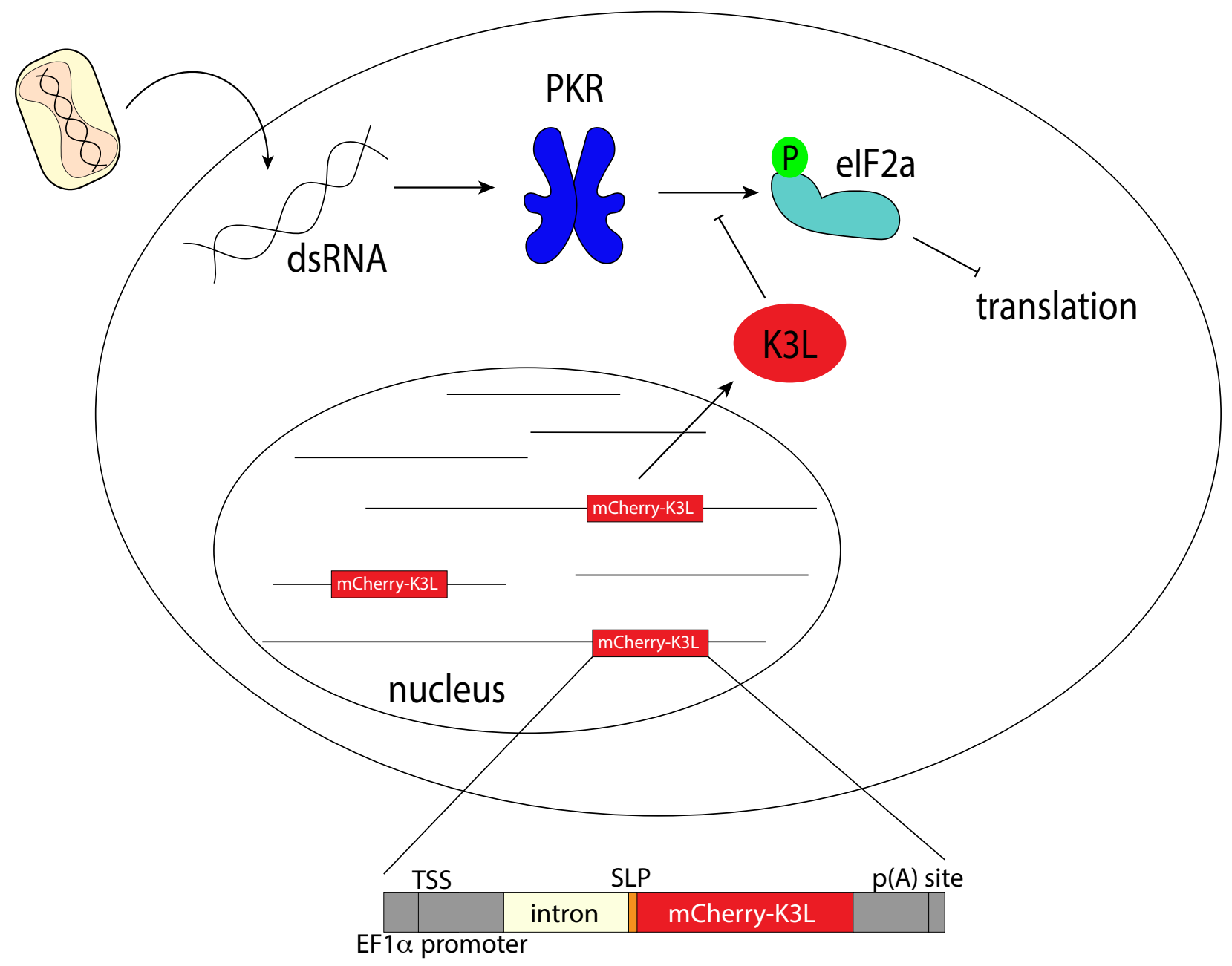

Fig. S2.

Protein Kinase R (PKR) is activated by binding dsRNA which is made in the cytoplasm during the course of a viral infection. When activated, PKR binds and phosphorylates the eukaryotic initiation factor, eIF2 $\alpha$, leading to a block in translation and preventing viral replication. K3L acts as a pseudo-substrate of PKR, preventing activated PKR from binding eIF2 $\alpha$, which is then free to initiate translation. In the complementing RK13-K3L cells, an mCherry-K3L fusion gene was integrated into cellular chromosomes. The cellular mCherry-K3L expression construct is shown below. The fusion gene is driven by a strong EF1a promoter, but the construct also includes a poxvirus-specific promoter, the SLP, so that mCherry-K3L could be immediately expressed if transferred. 


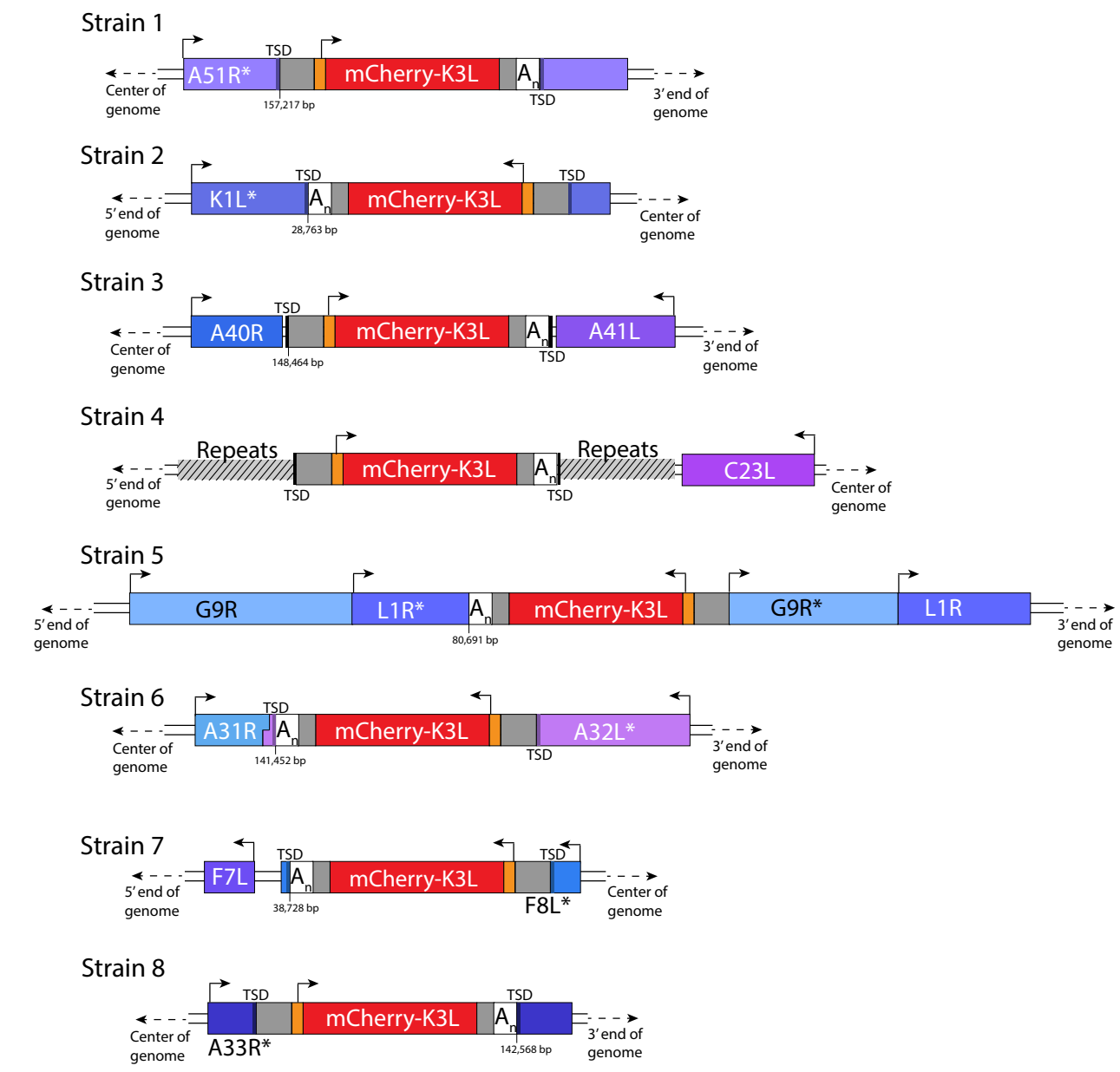

Strain 9

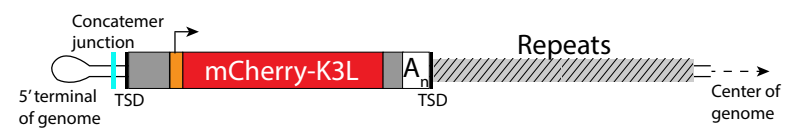

Strain $10(\sim 250 \mathrm{~kb})$

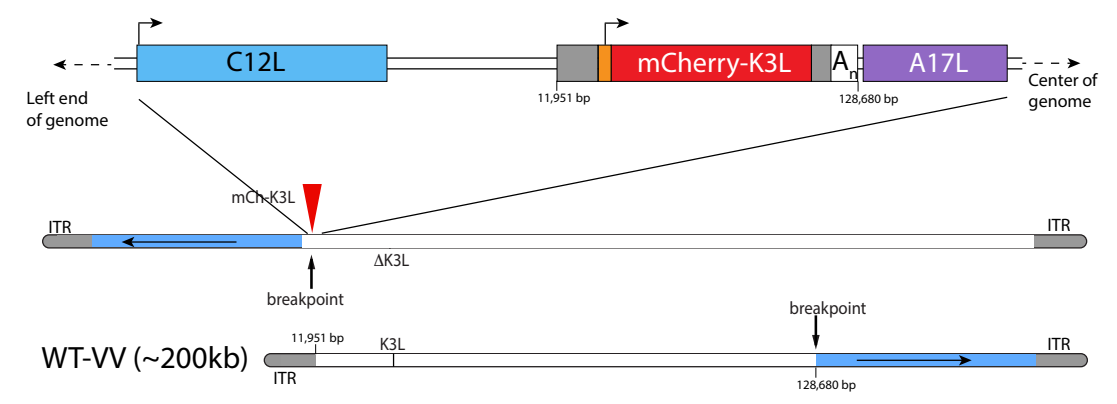

\section{Fig. S3.}

A detailed view of the region K3L inserted in each experimentally captured virus isolate. Arrows above cartoons indicate reading frame orientation. Flanking and/or interrupted $(*)$ viral genes (blue/purple boxes), 3'/5' untranslated regions (UTRs; gray), and poly(A) tails ( $\mathrm{A}_{\mathrm{n}}$; white) are shown. Genomic rearrangements included in isolate 10 are shown below details. Some isolate 4 viruses include a second insertion that precisely matches that of isolate 2 . We believe that this is due to recombination between the two strains during purification. 


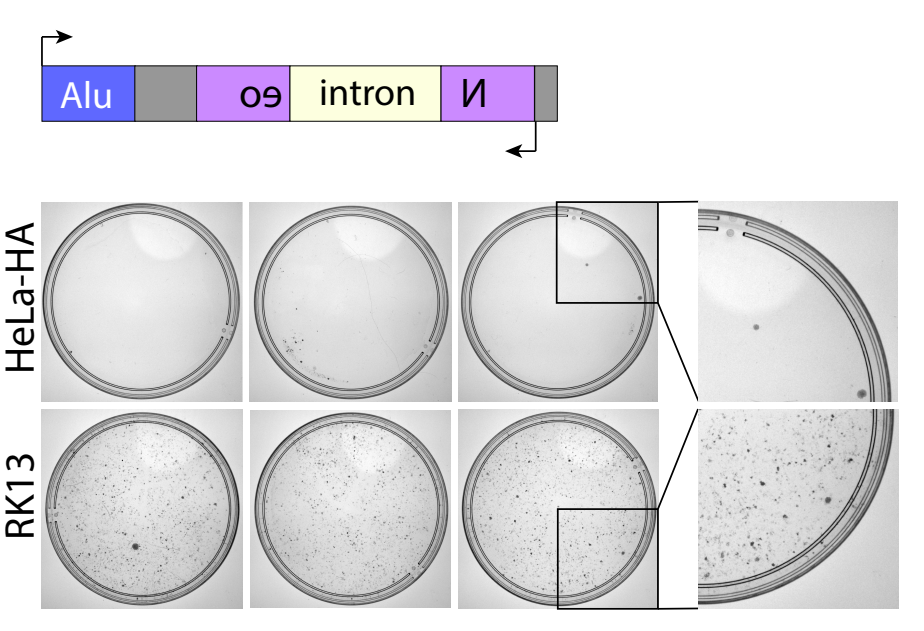

Fig. S4.

An Alu-Neo reporter (cartoon above) was used to interrogate the endogenous LINE-1 activity of the RK13-K3L cells compared to HeLa-HA cells. Below, representative plates from several experiments showing Neo-resistant colonies, with zoomed-in views to the right. 

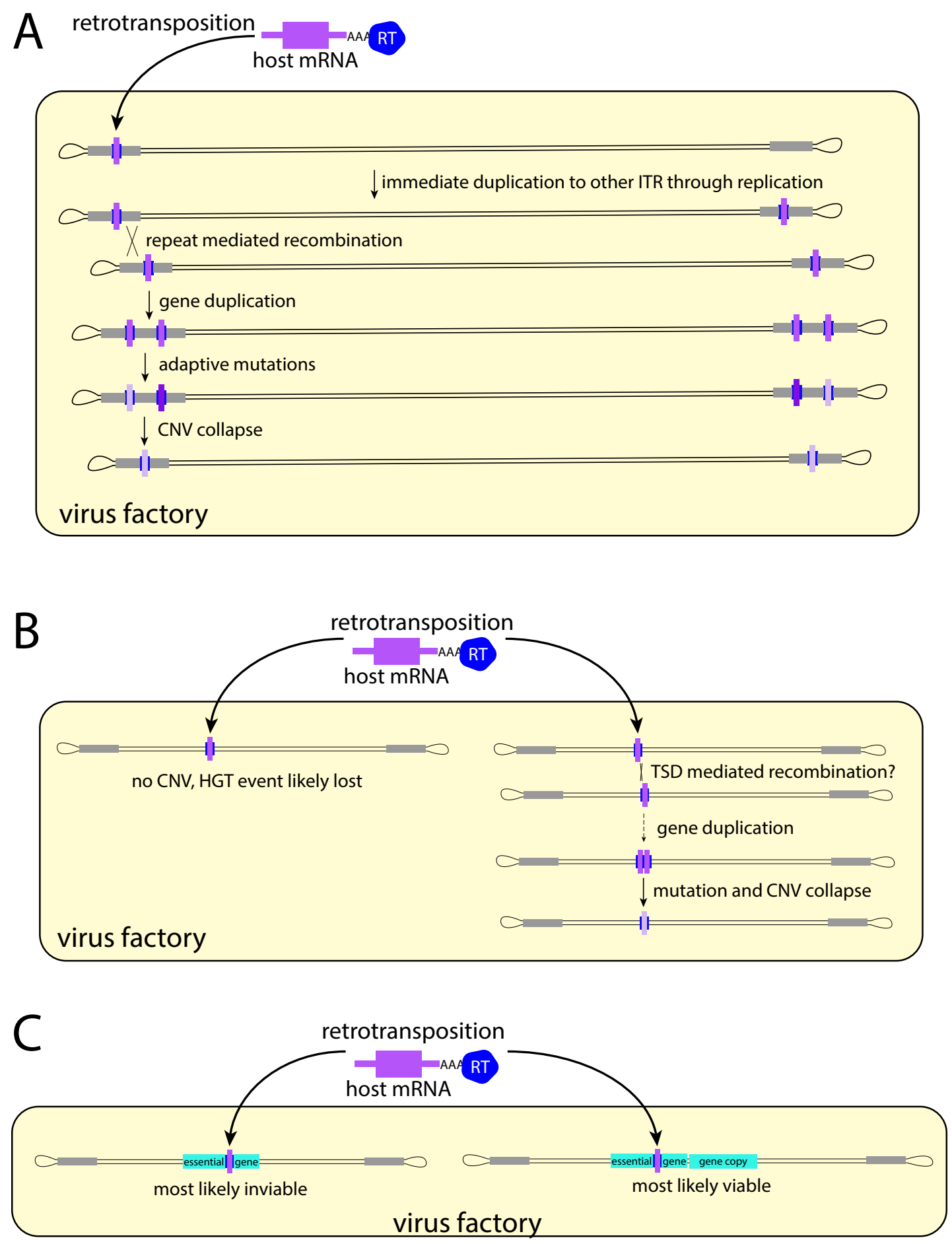

Fig. S5.

Models of evolution of newly acquired virus genes. (A) Genes that land in the repetitive region of the ITR are likely to be duplicated by both replication mechanisms (to the other ITR) and recombination mechanisms (tandem duplications). Duplications provide more chances for advantageous mutations to arise, increasing the likelihood of fixation. (B) Genes acquired outside the ITR may still be duplicated via TSD-mediated recombination. (C) Acquired genes that interrupt essential genes are unlikely to be maintained. 


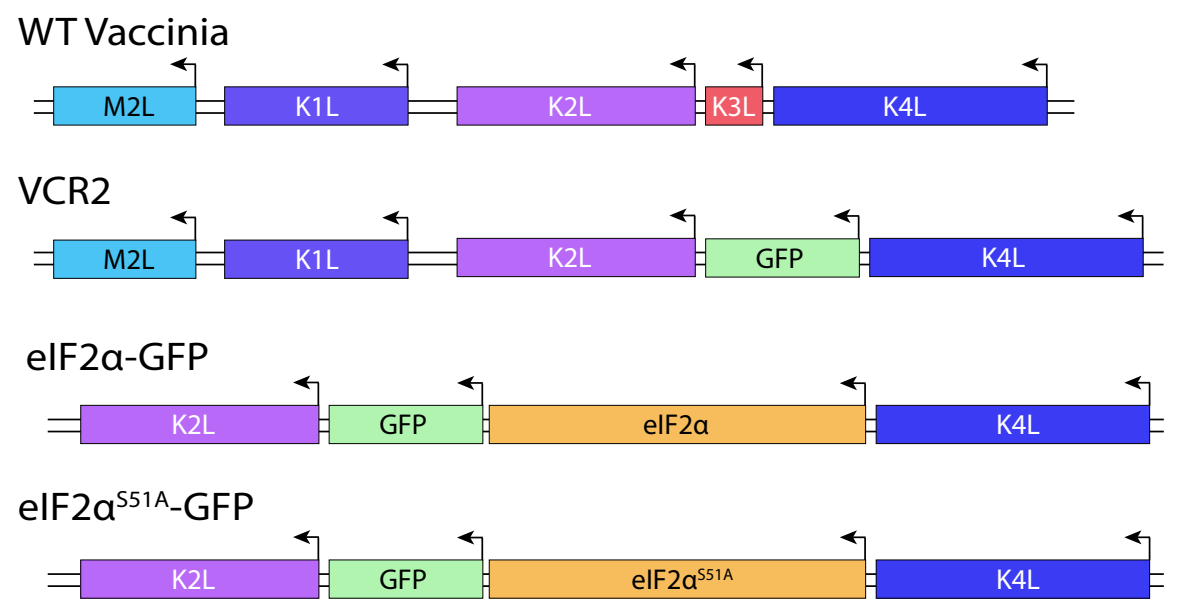

Fig. S6.

Structure of K3L region in eIF2 $\alpha$ vaccinia strains engineered for experimental evolution compared to wild type (WT) vaccinia and VCR2. 


\begin{tabular}{|c|c|c|}
\hline Organism & protein & Accession number \\
\hline Cowpox strain Ger14Cat1 & vGAAP/TMBIM4 & SNB58186.1 \\
\hline Cowpox strain Ger10Rac & vGAAP/TMBIM4 & SNB48400.1 \\
\hline Cowpox strain Ger02MKY & vGAAP/TMBIM4 & ADZ30397.1 \\
\hline Cowpox strain Ratpox09 & vGAAP/TMBIM4 & CRL86727.1 \\
\hline Cowpox strain Ger17vFME & vGAAP/TMBIM4 & SPN68348.1 \\
\hline Vaccinia strain EvansCDS & vGAAP/TMBIM4 & AAV98625.1 \\
\hline Cowpox strain Finland_2000_MAN & vGAAP/TMBIM4 & ADZ29327.1 \\
\hline Cowpox strain Kost $15 \mathrm{Hs}$ & vGAAP/TMBIM4 & AQQ13071.1 \\
\hline Vaccinia strain Lister & vGAAP/TMBIM4 & Q49P94.1 \\
\hline Cowpox strain Aus99cat & vGAAP/TMBIM4 & ADZ24213.1 \\
\hline Vaccinia strain USSR_CDS & vGAAP/TMBIM4 & AAW21699.1 \\
\hline Camelpox strain CMS & vGAAP/TMBIM4 & AAG37461.1 \\
\hline Camelpox strain Negev16 & vGAAP/TMBIM4 & QCW07309.1 \\
\hline Camelpox strain M96_Kazakstan & vGAAP/TMBIM4 & NP_570396.1 \\
\hline Cowpox strain GRI-90 & vGAAP/TMBIM4 & CAD90752.1 \\
\hline Cowpox strain FM2292 & vGAAP/TMBIM4 & CRL87022.1 \\
\hline Cowpox strain Ger07Vole & vGAAP/TMBIM4 & SNB49536.1 \\
\hline Cowpox strain Ger91-3 & vGAAP/TMBIM4 & ABD97561.1 \\
\hline Monkeypox strain USA03_044 & vGAAP/TMBIM4 & AAY96807.1 \\
\hline Monkeypox strain Cote d'Ivoire 1971 & vGAAP/TMBIM4 & AKG51345.1 \\
\hline Monkeypox strain DRC_07 & vGAAP/TMBIM4 & AGR36454.1 \\
\hline Monkeypox strain Zaire96 & vGAAP/TMBIM4 & NP_536611.1 \\
\hline Monkeypox strain Congo03 & vGAAP/TMBIM4 & AAY97191.1 \\
\hline Scale Drop Disease Iridovirus & TMBIM4 & YP_009163856.1 \\
\hline Harpseal herpes virus & TMBIM4 & AJG42933.1 \\
\hline Homo sapiens & TMBIM4 & NP_057140.2 \\
\hline Mus musculus & TMBIM4 & NP_080893.1 \\
\hline Rattus Norvigicus & TMBIM4 & NP_954547.1 \\
\hline Bos taurus & TMBIM4 & NP_001014914.1 \\
\hline Ovis aries & TMBIM4 & XP_014950252.2 \\
\hline Grizzly bear & TMBIM4 & XP_026355879.1 \\
\hline CA Sea lion & TMBIM4 & XP_027451274.1 \\
\hline Stellar sea lion & TMBIM4 & XP_027966737.1 \\
\hline
\end{tabular}




\begin{tabular}{|c|c|c|}
\hline Weddell seal & TMBIM4 & XP_006731256.1 \\
\hline Northern fur seal & TMBIM4 & XP_025738732.1 \\
\hline Ghost shark & TMBIM4 & NP_001279731.1 \\
\hline Bettafish & TMBIM4 & XP_029017613.1 \\
\hline Yellow perch & TMBIM4 & XP_028426613.1 \\
\hline Gilt head Bream & TMBIM4 & XP_030296356.1 \\
\hline Homo sapiens & TMBIM3 & NP_000828.1 \\
\hline Mus musculus & TMBIM3 & NP_075657.1 \\
\hline Rattus Norvigicus & TMBIM3 & NP_695220.4 \\
\hline Bos taurus & TMBIM3 & NP_001032682.1 \\
\hline Ovis aries & TMBIM3 & NP_001265493.1 \\
\hline Grizzly bear & TMBIM3 & XP_026344576.1 \\
\hline CA Sea lion & TMBIM3 & XP_027465497.1 \\
\hline Weddell seal & TMBIM3 & XP_006745469.1 \\
\hline Northern fur seal & TMBIM3 & XP025727080.1 \\
\hline Whale shark & TMBIM3 & XP020385145.1 \\
\hline Bettafish & TMBIM3 & XP_029023514.1 \\
\hline Yellow perch & TMBIM3 & XP_028453020.1 \\
\hline Gilt head Bream & TMBIM3 & XP_030268118.8 \\
\hline Homo sapiens & TMBIM2 & NP_036438.2 \\
\hline Mus musculus & TMBIM2 & NP_082500.2 \\
\hline Rattus Norvigicus & TMBIM2 & NP_653357.1 \\
\hline Bos taurus & TMBIM2 & NP_001068886.1 \\
\hline Ovis aries & TMBIM2 & XP_0040066421.1 \\
\hline Grizzly bear & TMBIM2 & XP_026357704.1 \\
\hline CA Sea lion & TMBIM2 & XP_027450780.1 \\
\hline Weddell seal & TMBIM2 & XP_006729299.1 \\
\hline Northern fur seal & TMBIM2 & XP_025739188.1 \\
\hline Whale shark & TMBIM2 & XP_020373190.1 \\
\hline Bettafish & TMBIM2 & XP_029006890.1 \\
\hline Yellow perch & TMBIM2 & XP_028437735.1 \\
\hline Atlantic sea bass & TMBIM2 & XP_018521446.1 \\
\hline Gilt head bream & TMBIM2 & XP_030278166.1 \\
\hline Danio rerio & TMBIM2 & XP_005163554.1 \\
\hline Homo sapiens & TMBIM1 & NP_001308356.1 \\
\hline
\end{tabular}




\begin{tabular}{|c|c|c|}
\hline Mus musculus & TMBIM1 & NP_081430.3 \\
\hline Rattus Norvigicus & TMBIM1 & NP_001007714.1 \\
\hline Bos taurus & TMBIM1 & NP_991367.1 \\
\hline Ovis aries & TMBIM1 & XP_004004971.1 \\
\hline CA Sea lion & TMBIM1 & XP_027443873.1 \\
\hline Weddell seal & TMBIM1 & XP_006750605.1 \\
\hline Northern fur seal & TMBIM1 & XP_025747486.1 \\
\hline Ghost shark & TMBIM1 & XP_007882730.1 \\
\hline Bettafish & TMBIM1 & XP_028986890.1 \\
\hline Yellow perch & TMBIM1 & XP_028448525.1 \\
\hline Atlantic sea bass & TMBIM1 & XP_018532965 \\
\hline Gilt head bream & TMBIM1 & XP_030257449.1 \\
\hline Danio rerio & TMBIM1 & NP_001005992.2 \\
\hline Homo sapiens & TMBIM5 & NP_055209.2 \\
\hline Mus musculus & TMBIM5 & NP_001186051.1 \\
\hline Rattus Norvigicus & TMBIM5 & NP_001005908.1 \\
\hline Bos taurus & TMBIM5 & NP_001029224.1 \\
\hline Ovis aries & TMBIM5 & XP_004021578.1 \\
\hline Grizzly bear & TMBIM5 & XP_026360365.1 \\
\hline CA Sea lion & TMBIM5 & XP_027452399.1 \\
\hline Monk seal & TMBIM5 & XP_021555821.1 \\
\hline Northern fur seal & TMBIM5 & XP_025736444.1 \\
\hline Ghost shark & TMBIM5 & AFK1 1640.1 \\
\hline Bettafish & TMBIM5 & XP_028990283.1 \\
\hline Yellow perch & TMBIM5 & XP_028423643.1 \\
\hline Atlantic sea bass & TMBIM5 & XP_18554801.1 \\
\hline Danio rerio & TMBIM5 & NP_956885.1 \\
\hline Homo sapiens & TMBIM6 & NP_003208.2 \\
\hline Mus musculus & TMBIM6 & NP_001164505.1 \\
\hline Rattus Norvigicus & TMBIM6 & NP_062254.2 \\
\hline Bos taurus & TMBIM6 & NP_001069882.1 \\
\hline Ovis aries & TMBIM6 & XP_004006423.2 \\
\hline Grizzly bear & TMBIM6 & XP_026357713.1 \\
\hline CA Sea lion & TMBIM6 & XP_027449007.1 \\
\hline Weddell seal & TMBIM6 & XP_006729302.1 \\
\hline Northern fur seal & TMBIM6 & XP_025738444.1 \\
\hline
\end{tabular}


bioRxiv preprint doi: https://doi.org/10.1101/2020.10.26.356402; this version posted October 27, 2020. The copyright holder for this preprint (which was not certified by peer review) is the author/funder, who has granted bioRxiv a license to display the preprint in perpetuity. It is made available under aCC-BY-NC-ND 4.0 International license.

$\begin{array}{llc}\text { Ghost shark } & \text { TMBIM6 } & \text { XP_007910207.1 } \\ \text { Bettafish } & \text { TMBIM6 } & \text { XP_029013526.1 } \\ \text { Yellow perch } & \text { TMBIM6 } & \text { XP_028439299.1 } \\ \text { Atlantic sea bass } & \text { TMBIM6 } & \text { XP_018531358.1 } \\ \text { Gilt head bream } & \text { TMBIM6 } & \text { XP_030277962.1 }\end{array}$

Table S1.

Accession numbers for sequences used in phylogenetic analysis (Fig. 1B)

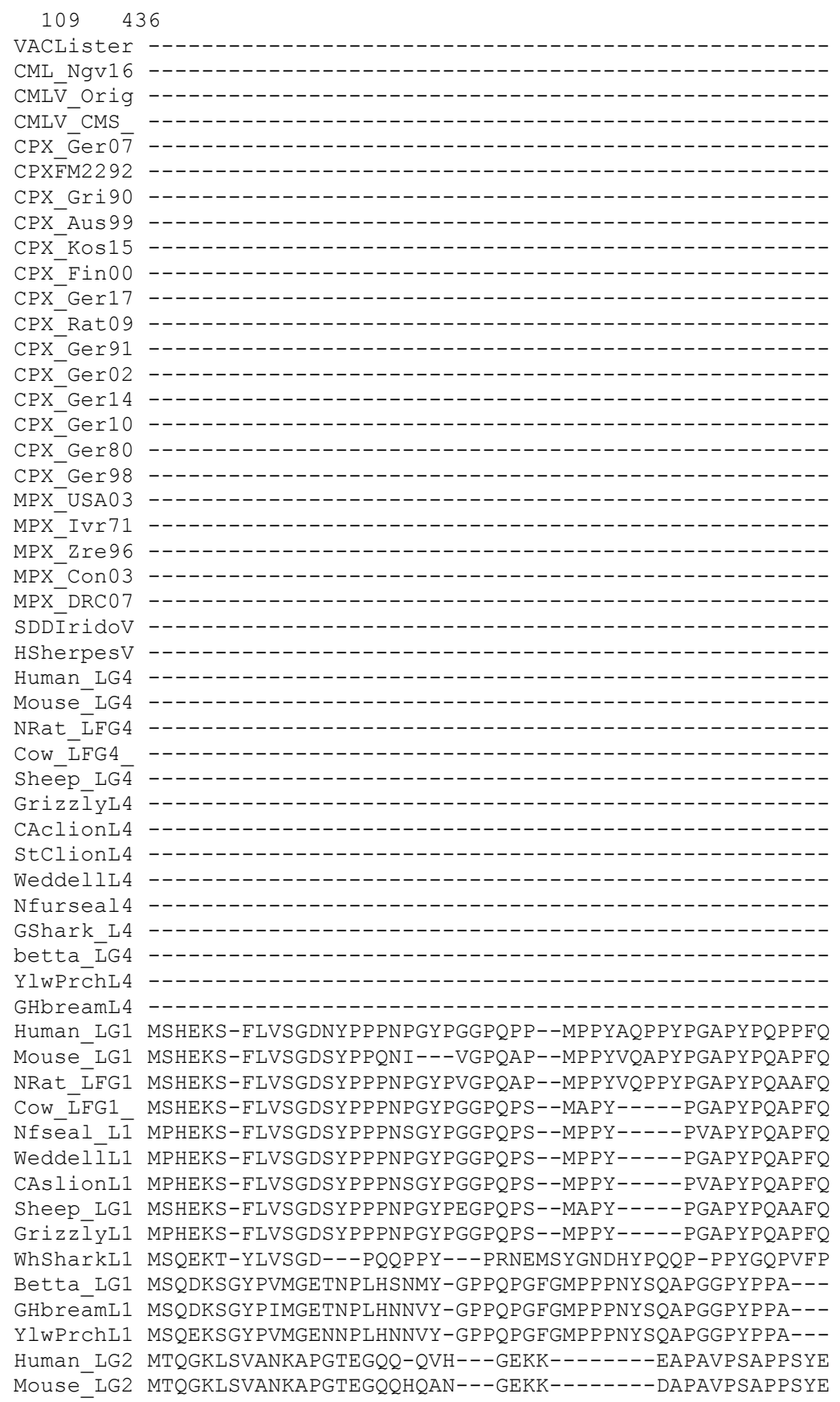


bioRxiv preprint doi: https://doi.org/10.1101/2020.10.26.356402; this version posted October 27, 2020. The copyright holder for this preprint (which was not certified by peer review) is the author/funder, who has granted bioRxiv a license to display the preprint in perpetuity. It is made available under aCC-BY-NC-ND 4.0 International license.

NRat LFG2 MTQGKLSVANKAPGTEGQQ-QAN---GEKK--------DAPAVPSAPPSYE COW LFG2 MTQGKLSVANKAPGTEGQQ-QAN---GEKK--------ETPAVPSAPPSYE Sheēp_LG $\overline{2}$ MTQGKLSVANKAPGTEGQQ-QAN---GEKK--------ETPAVPSAPPSYE GrizzIyL2 MTQGKISVANKAPGTEGQQ-QAN---GEKK--------ETPAVPSAPPSYE WeddellL2 MTQGKISVANKAPGTEGQQ-QAN---GEKK--------ETPAVPSAPPSYE Nfseal L2 MTQGKISVANKAPGTEGQQ-QAN---GEKK--------ETPAVPSAPPSYE CAslioñ2 MTQGKISVANKAPGTEGQQ-QAN---G---------------------WhSharkL2

betta_LG2 MKKGKVSDANE--------------------------------PPSYQ Asbass L2 MTQGKLSLANKA--TNGSS---S---GQAL--------VAPA----PPSYE Ylwprh_L2 MTKGKLSLANKA--TNGSF---S---EEAL--------VSPA----PPSYQ GHbreamL2 MTQGKLSLANKS---NDSS---S---GQAL--------GTPS----PPSYE

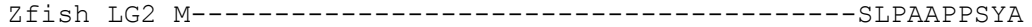
Human_LG3 MSNPSAPPPYED-RNPL-------YPGP---PPPGGYGQPSVLPGGYPAYP Mouse LG3 MSNPSAPPPYED-HNPL-------YPGS---PPPGGYGQPSVLPGGYPAYP NRat_LFF3 MSNPTAPPPYED-HNPL-------YPGR---PPPGGYGQPSVLPGGYPAYP COW LFG3 MSHPSAPPPYED-RSPL-------YPGS---PPPGGYGQPSVMPGGYP--Sheep LG $\overline{3}$ MSHASAPPPYED-RSPL-------YPGS---LPPGGYGQPSVVPGGYP--NfseaI L3 MSNPSAPPPYED-RNPL-------YPGS---PPQGGYAQPSVLPGGYPPYP CAslionL3 MSNPSAPPPYED-RNPL-------YPGS---PPQGGYAQPSVLPGGYPPYP WeddellL3 MSNPSAPPPYED-RNPL-------YPGS---PPQGGYAQPFVLPGGYP--GhSharkL3 MSRPTAPPSYEEATDLVMLPVHGEYPGNTVYPLPGSY-----SPTGKPGYP Betta LG3 MSRSDYPPGYEDCHGPL------YP-----PQGGNYPAP-PPYGFPGYAsbass L3 MSRSDYPPGYDDSHGPL-------YA------PQGGSYPPP-PAYGFPAYGHbream̄3 MSRSDYPPGYDDSRGPM-------YA------PQGGNYPPP-PAYGFPAYYlwPrchL3 MSRSDYPPGYDDSRDLL-------YA------PQGGNYPPP-PAYGFPGFZfish_LG3 MSRSDFPPSYDDSRLLS-------NT------QPGPGYPPSAPPYGFSPYHuman TB5 MLAARLVCLRTLPSRVFHPAF-T--------------KASPVVKNSIT Mouse_TB5 MLAARLVCLRTLPSRVFQPTFIT--------------KASPLVKNSIT Rat TBIM5 MLAARLVCLRTLPSRVFQPTFIT---------------KASPLVKNSIT COW_TBIM5 MLAARLVCLRALPSRVFHPAF-T---------------KASPVVKNSIT Sheēp TB5 MLAARLVCLRALPSRVFHPAF-T--------------KASPAVKNSIT GrizzİT5 MLAARLLCLRTLPSRVFQPAF-T---------------KASPIVKNSIT Nfseal_T5 MLAARFMCLRTLPSRVFQPAF-T---------------KASPIVKNSIT CAslionT5 MLAARFMCLRTLPSRVFQPAF-T---------------KASPIVKNSIT HIMonkST5 MLAARLVCLRTLPSRVFQPAF-T----------------KASPIVKNSIT GhSharkT5 MLVARFACLRNLPSLSLRPGL-T---------------QGSVAWRNPSQ betta_TB5 MLVARLTCLRSLPLAGMRPVL-T----------------HGSPALRAPTL YlwPrchT5 MLVARLTCLRSLPLAGLRPVL-S---------------QGSPALRAPTL Asbass T5 MLVARLTCLRSLPLAGLRPVL-T---------------QGSPALRTSTL Zfish TB5 MLLCRLSCLRA-------PLGLR--------------CAVPQGPRALI

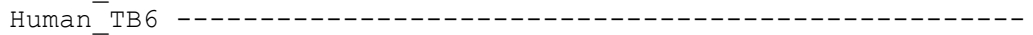
Mouse TB6 --.--

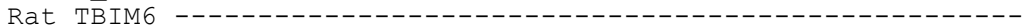

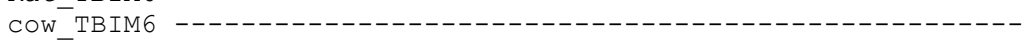

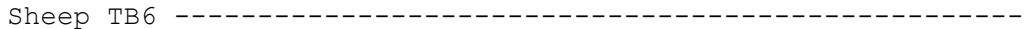

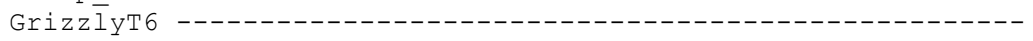

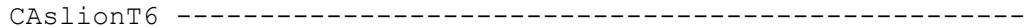

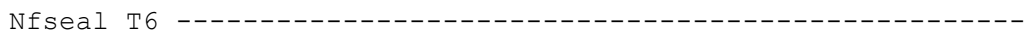

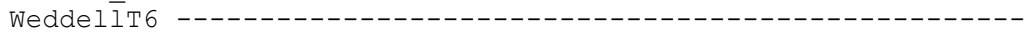

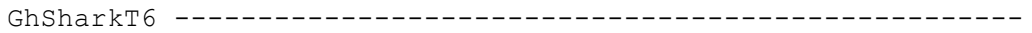

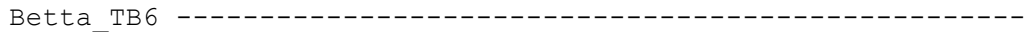
YlwPrchT6 --.--

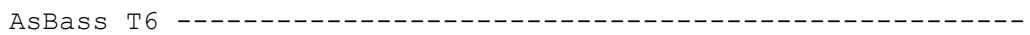

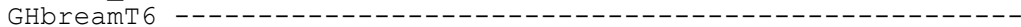

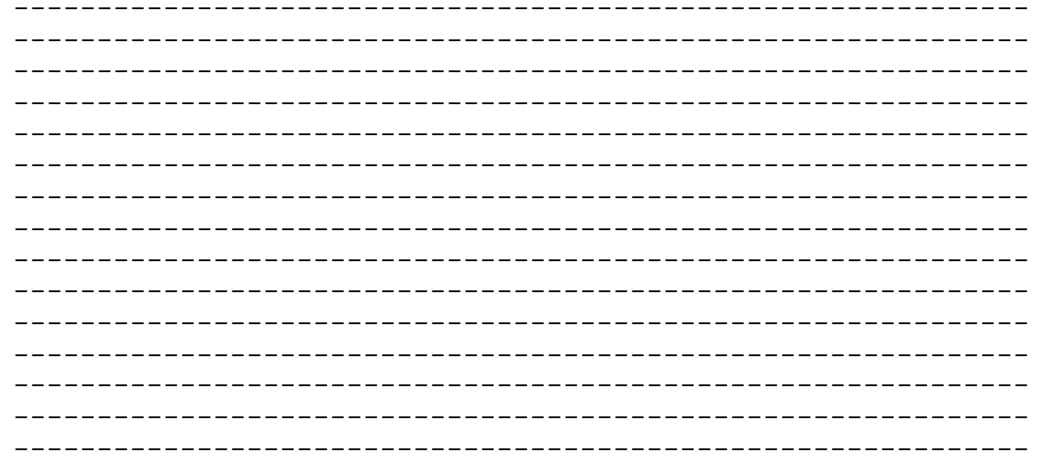


bioRxiv preprint doi: https://doi.org/10.1101/2020.10.26.356402; this version posted October 27, 2020. The copyright holder for this preprint (which was not certified by peer review) is the author/funder, who has granted bioRxiv a license to display the preprint in perpetuity. It is made available under aCC-BY-NC-ND 4.0 International license.

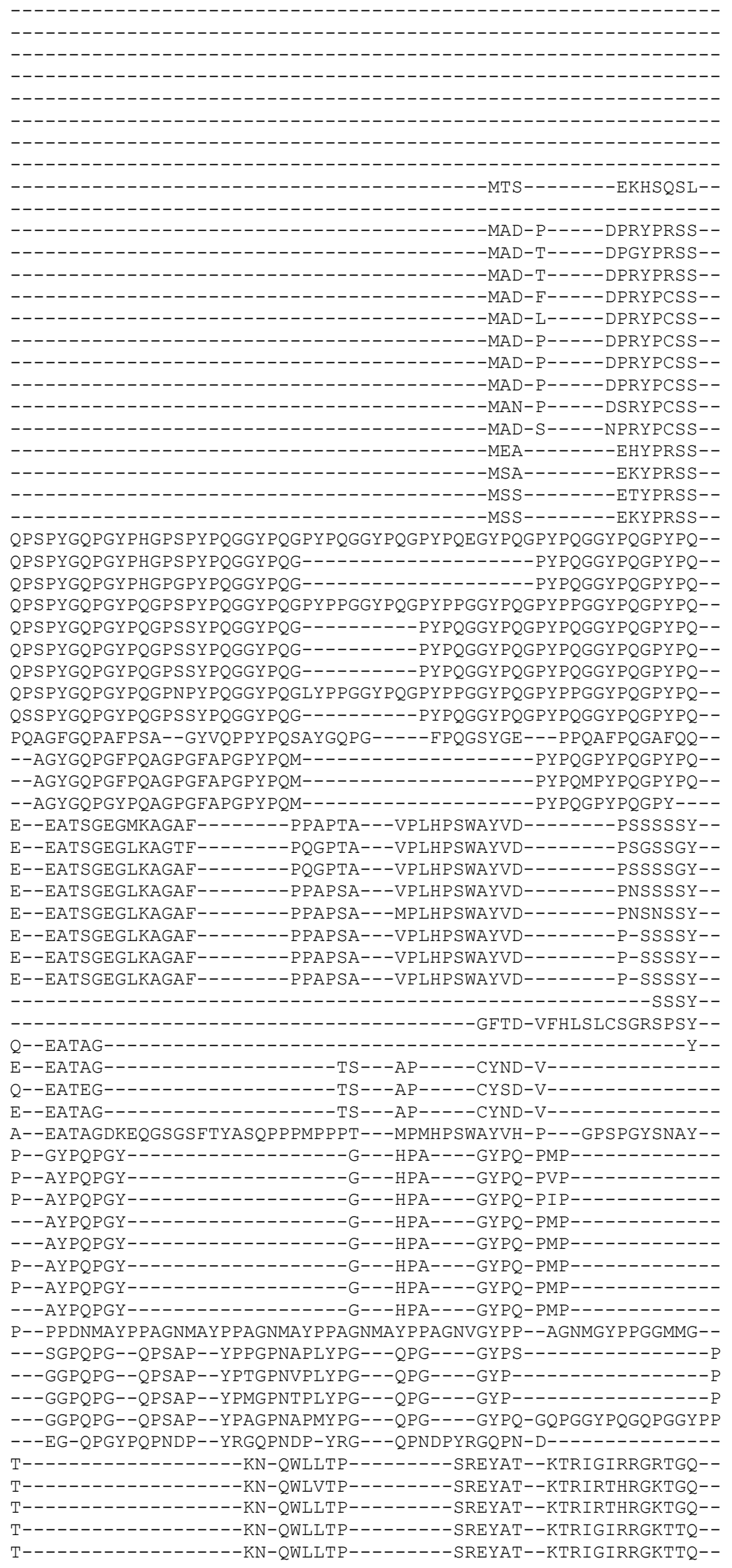


bioRxiv preprint doi: https://doi.org/10.1101/2020.10.26.356402; this version posted October 27, 2020. The copyright holder for this preprint (which was not certified by peer review) is the author/funder, who has granted bioRxiv a license to display the preprint in perpetuity. It is made available under aCC-BY-NC-ND 4.0 International license.

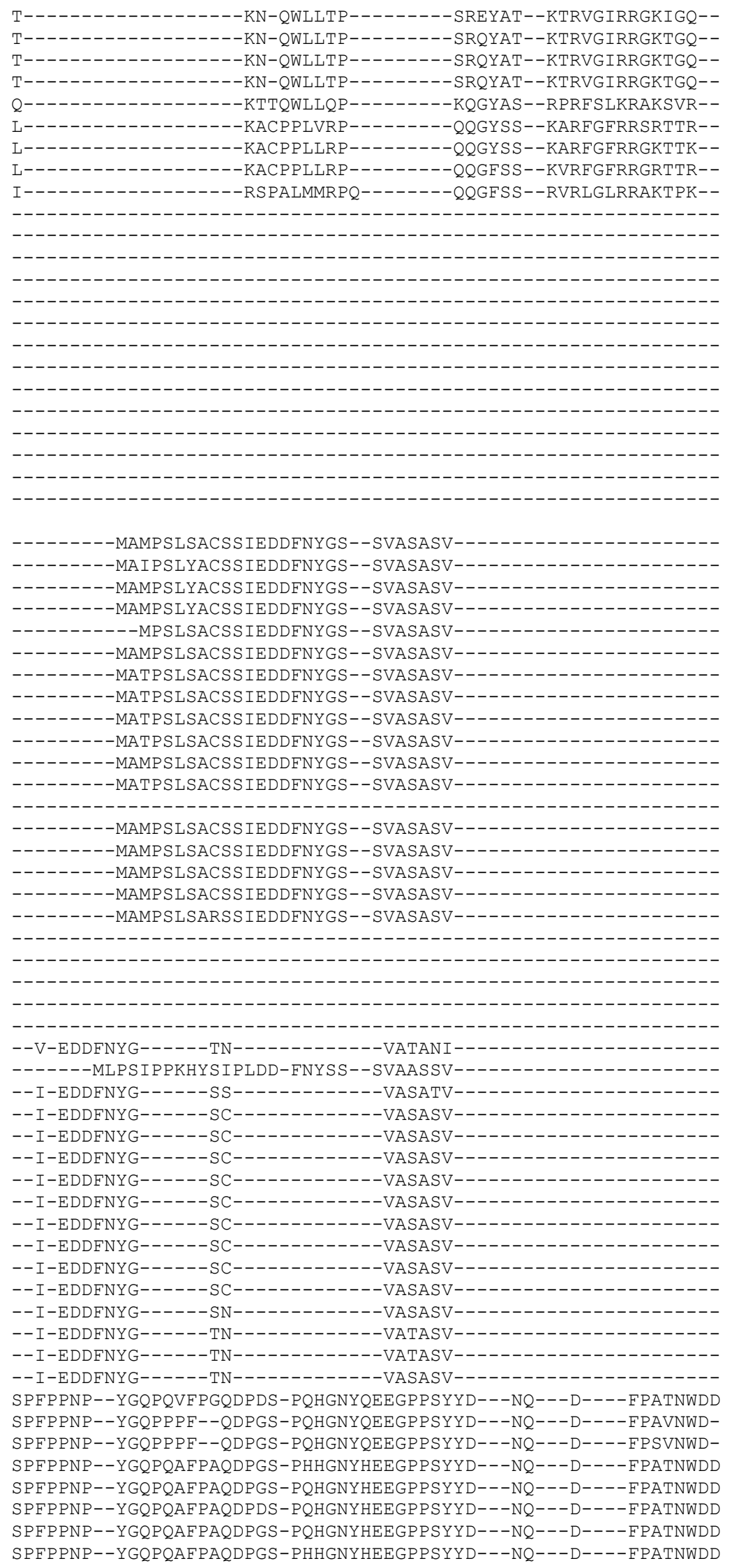


bioRxiv preprint doi: https://doi.org/10.1101/2020.10.26.356402; this version posted October 27, 2020. The copyright holder for this preprint (which was not certified by peer review) is the author/funder, who has granted bioRxiv a license to display the preprint in perpetuity. It is made available under aCC-BY-NC-ND 4.0 International license.

SPFPPNP--YGQPQAFPAQDPGS-PQHGNYHEEGPPSYYD---NQ---D----FPATNWDD PPMPPPP----------MMHSNVALGSPGYHDDVPPSYYD---NE---E----FSAANFED GPYPQGPFQQGPAQPGFLGDPTGPATSPSYHGEGPPSYYD---NE---E----FTGSGFED GPY-----QQGPGQPGFPGDPTVPAGSPGYHGDGPPSYYD---NE---E----FTNSGFED -------QQGVAQPGFLSDPSA-VGSAGYHGDVPPSYYD---NE---E----FTNSGFED

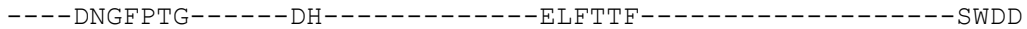
---- EGGEPAG------HH-----------EHFTTF----------------SWDD

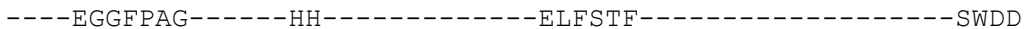

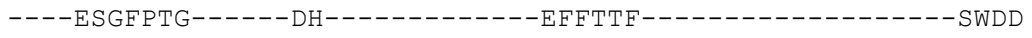

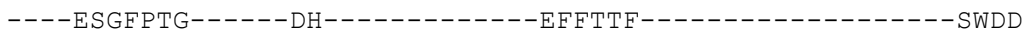
---- ENGFPAG------DH------------ELFTTF---------------- SWDD ---- ENGFPAG------DH-----------ELFTTF----n---n--------SWDD ---- ENGFPAG------DH----------- ELFTTF-----------------SWDD ---- ENGFPAG------DH-----------ELETTF----------------SWDD ---- GSGSFSG------ES------------ ELFSSF------------------SWDD

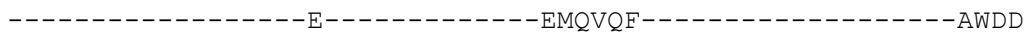

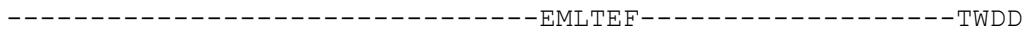

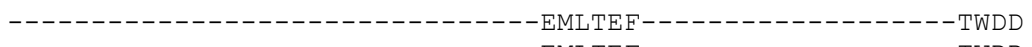
---------------------------- EMLTEF---------------- TWDD -- AADMSSPFS------DP-------------SSSNSFDGLGS--------------NWED - PTHPMPMNYG-PGHGYDG--------E--ERAVSDSFGPG-------E---------WDD -PVHPMPMNYG---HDYNE--------E--ERAGSDSFRPG-------E---------WDD -PVHPMPMNYG---HDYSE--------E--ERAGSDSFGPG-------E---------WDD -PVHPMPMNYG-AGHGYDG--------G--ERAVSDTFGSG-------D--------WDD -PVQPMPMNYG-PGHGYDG--------G--ERAVSDTFGSG-------D---------WDD -PIHPMPMRYG-PGQGYDG--------E--ERAVSESFGPG-------E-------- WDD -PIHPMPMRYG-PGQGYDG--------E--ERAVSESFGPG-------E---------WDD -PIHPMPMRYG-PGQGYDG--------E--ERAVSESFGPG-------E--------WDD ---APPAGSPGYPGGGFPIPPTLPLNPSW-PGPGSGPYGSSGSST-HPESE-GFTSSSWST GPYPGQPYPPGHPGAGYPS-PPMPPVMP--PTIPTDVMSSG-------DDEFAASGSGWDS GPYQGQPHPAGP PGAGYP--PPMP PVVP--PTIPSDVLSSG-------D-GFAASGSGWDS GPYSGQPHPGGHPGAGYPSPPPMPPVIP--PTIPSDVLSSG-------D-EFAAHGSGWDS GPYPGQPHPGGPPGAGYPSRPPMPPVMP--PTMPSDILSSG------E-EFAASDSSWDS -PYRGQP-------AGYP--PPAMPVIP--VMPPPGI---G-------DSEGETTAEGEES E-LKEAALEPSMEKIFKI DQMGRWFVAGG----AAVGLGALCYYGLGLSNEIGAIEKAVIW E-LKEAALEPSMEKIFKI DQMGRWFVAGG----AAVGLGALCYYGLGMSNEIGAIEKAVIW E-LKEAALEPSLEKVFKI DQMGKWFVAGG----AAVGLGALCYYGLGMSNEIGAIEKAVIW E-LKEAALEPSVEKIFK I DQMGRWFIAGG----AAVGLGALCYYGLGMSNEIGA IEKAVIW E-LKEAALEPSVEKIFKI DQMGRWFIAGG----AAVGLGALCYYGLGMSNEIGAIEKAVIW E-LKEAAMEPSVEKIFKI DQMGRWVIAGG----AAVGLGALCYYGLGMSNEIGAIEKAVIW E-LKEAAMEPSMEKIFK I DQMGRWFIAGG----AAVGLGALCYYGLGMSNEIGAIEKAVIW E-LKEAAMEPSMEKI FK I DQMGRWFIAGG----AAVGLGALCYYGLGMSNEIGAIEKAVIW E-LKEAAMEPSVEKI FK I DQMGRWFIAGG----AAVGLGALCYYGLGMSNEIGAIEKAVIW EDFKDAAYQPAWETAAKMHNTGKLFVAGG----AAVGLGALCYYGLGMSNEIGAIEKAVIW DQMKDAAFEPATDTAIRIDSMGRI I LAGG----AAVGLGALCYYGLGMSNEMGAIEKAVIW DQLKEAAFEPATDTAIRIDGMGRMVLAGG----AAVGLGALCYYGLGMSNEIGAIEKAVIW DQLKEAAFEPATDTAIRIDSMGRMI LAGG----AVVGLGALCYYGLGMSNDIGAIEKAMIW EQLQDAALQ-NTHTASRVDSMGRVLLAGG----AAVGLGALCYYGLGMSSEIGAIEKAAIW -------------------------MNIFDRKINF---D----ALLKFSHI ------------------------------MNI F DRKINF---D----ALLKFSHI $-----------------------------M N I F D R K I N F---D----$ ALLKF SHI

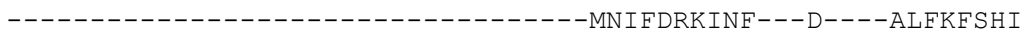
$-------------------------M N I F D R K I N F---D----A L F K F$ SH I

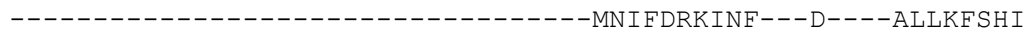

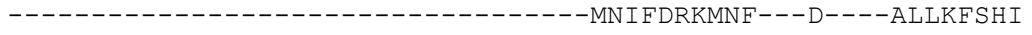
----------------------------MNIFDRKINF---D----ALLKFSH I --------------------------MNIFDRKINF---D----ALLKF SH I

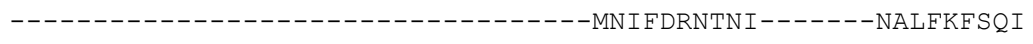
---

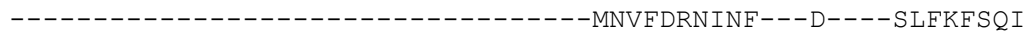
--------------------------MNVFDRNINF---D----ALFKF SQI

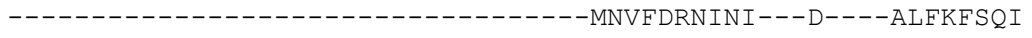

-HIRMAFLRKVYGILCLQFLLTTATTAVFLYFDCMRTFIQGSPVLIL---ASMFGSIGL--HIRMAFLRKVYGILCLQFLLTTATTAVFLYFDCMRKF IQGS PVLIL---ASMFVSIGL--HIRMAFLRKVYGI LCLQFLLTTATTAVFLYFDCMRKI IQGSPVLIL---ASMFVSIGL--H I RMAFLRKVYGI LCLQFLLTTATTAVFLYFDCMRKFIQGSPVLIL---ASMFVSIGL--HIRMAFLRKVYGI LCLQFLLTTATTAVFLYFDCMRTFIQGSPVLIL---ASMFGSIGL--HIRMAFLRKVYGI LCLQFLLTTATTAVFLYFDCMRTFIQGSPVLIL---ASMFGSIGL--HIRMAFLRKVYGI LCLQFLLTTATTAVFLYFDCMRTFIQGSPVLIL---ASMFGSIGL--HIRMAFLRKVYGILCLQFLLTTATTAVFLYFDCTRTFIQGSPVLIL---ASMFGSIGL-- 
bioRxiv preprint doi: https://doi.org/10.1101/2020.10.26.356402; this version posted October 27, 2020. The copyright holder for this preprint (which was not certified by peer review) is the author/funder, who has granted bioRxiv a license to display the preprint in perpetuity. It is made available under aCC-BY-NC-ND 4.0 International license.

-HIRMAFLRKVYGI LCLQFLLTTATTAVFLYFDCMRTFIQGSPVLIL---ASMFGSIGL--HIRMAFLRKVYGILCLQFLLTTATTAVFLYFDCMRTFIQGS PVLIL---ASMFGSIGL--HIRMAFLRKVYGILCLQFLLTTATTAVFLYFDCMRTFIQGSPVLIL---ASMFGSIGL--HIRMAFLRKVYGILCLQFLLTTATTAVFLYFDCMRTFIQGSPVLIL---ASMFGS IGL-----MAFLRKVYGILCLQFLLTTATTAVFLYFDCMRTFIQGSPVLIL---ASMFGSIGL--H IRMAFLRKVYGI LCLQFLLTTATTAVFLYFDCMRTF I QVSPVLIL---ASMFGS IGL--HIRMAFLRKVYGILCLQFLLTTAMTAVFLYFDCMRTFIQGSPVLIL---ASMFGS IGL--H IRMAFLRKVYGI LCLQFLLTTATTAVFLYFDCMRTF IQGS PVLIL---ASMFGSIGL--HIRMAFLRKVYGILCLQFLLTTATTAVFLYFDCMRTFIQGSPVLIL---ASMFGS IGL--HIRMAFLRKVYGILCLQFLLTTATTAVFLYFDCMRTFIQGSPVLIL---ASMFGSIGL---------------------------MHRTF I QGS PVL I L---ASMFGS I GL --- - - - - - ---

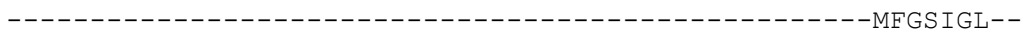
-KIRMTFIRRVYTLLSLQIILTISTTALFMFSKHLNAFVQDNSAVVF---VSAFSSFV---HIQLAFLRRVYGI LLLQLLLTALVC I LFLCF P INTFLCTYPS IMA--- ICP I I SMVL--HIRMAFLRKVYSILSLQVLLTTVTSTVFLYFESVRTFVHESPALIL---LFALGSLG---HIRMAFLRKVYS I LSLQVLLTTVTSALFLYFQALRTFVHESPALIV---VFALGSLG---HIRMAFLRKVYS I LSLQVLLTTVTSALFLYFETLRTFVHDSPALIV---VFALGSLG---HIRMAFLRKVYS I LSLQVLLTTVTLAFFLYFDS IRTFVHESPALIL---VLALGSLG---H IRMAFLRKVYS ILSLQVLLTTVTSAIFLYFDS IRTFVHESPALIL---VLTLGSLG---HIRMAFLRKVYS I LSLQVLLTTVTASFFLYFES IRTFVHES PAL IL---VFALGSLG---HIRMAFLRKVYS I LSLQVLLTTVTASFFIYFES IRTFVHESPALIL---VFALGSLG---HIRMAFLRKVYS I LSLQVLLTTVTASFFIYFES IRTFVHESPALIL---VFALGSLG---HIRLAFLRKVYS I LSLQVLLTTVTASFFLYFES IRTFVHESPALIL---MFALGSLG---HIRMAFLRKVYS I LS LQVLLTTVTASFFIYFES IRTFVHESPALIL---VFALGSLG---HIRMAFLRKVYTILSLQI ILTTVTSAVFMYSDTIKDFI HTSPAFVL---VPALGSLG---QIRMDFLRKVYTLLSLQI ILTTATSAVFMFSQTIKDFVHSSPAVVL---VSALGSLV---QIRMDFLRKVYTLLSLQI ILTTATSALFMFSQPIKEFVHASPAVVL---VSALGSLV---QIRMDFLRKVYTLLSLQI ILTTATSALFMFSHTIKEFVHAS PAMVL---VSALGSLV--KS IRQAFIRKVFLVLTLQLSVTLSTVSVFTFVAEVKGFVRENVWTYY---V----SYA--KNIRQAFIRKVFLVLTLQLSVTLSTVAIFTFVGEVKGFVRENVWTYY---V----SYA--KS IRQAFIRKVFLVLTLQLSVTLSTVA IFTFVGEVKGFVRANVWTYY---V----SYA--KS IRQAFIRKVFLVLTLQLSVTLSTVAVFTFVGEVKGFVRENVWTYY---V----SYA--KS IRQAFIRKVFLVLTLQLSVTLSTVAVFTFVGKVKGFVRENVWTYY---V----SYA--KS IRQAFIRKVFLVLTLQLSVTLSTVAVFTFVGKVKGFVRENVWTYY---V----SYA--KS IRQAFIRKVFLVLTLQLSVTLSTVAVFTFVGKVKGFVRENVWTYY---V----SYA--KS IRQAFIRKVFLVLTLQLSVTLSTVAVFTFVGEVKGFVRENVWTYY---V----SYA--KSIRQAFIRKVFLVLTLQLSVTLSTVAVFTFVGKVKGFVRENVWTYY---V----SYA--KNIRRAFIRKVYMVLTVQLLVTF I FVCICTFVSDAKKFVRRTPWIYY---A----SYG--KNIRQAFIRKVFMVLTVQLLVTFSFVAVFTFVDDAKYFVRRNPWTYY---V----SYA--KNIRQAFIRKVFLVLTVQLMVTFSFVAVFTFVDEAKDFVRKNPWTYY---V----SYA--KS IRQAFIRKVFMVLTVQLMITFSFVAVFTFVNEAKFFVRRNPWTYY---V----SYA--QKVRRVFVRKVYTILLIQLLVTLAVVALFTFCDPVKDYVQANPGW-------YWASYA--QKVRRLFIRKVYTILLVQLLVTLAVVALFTFCDVVKDYVQANPGW-------YWASYA--QKVRQLFIRKVYTI LLVQLLVTLAVVALFTFCDVVKDYVQANPGW-------YWASYA--QKVRRVFIRKVYTILLIQLLVTLGVVALFTFCDPVKDYVQANPGW-------YWASYA--QKVRRVFIRKVYTILLIQLLVTLGVVALFTFCDPVKDYVQANPGW-------YWASYA--QKVRRVFIRKVYTI LLIQLLVTLGVVALFTFCDPVKDYVQANPGW-------YWASYA--QKVRRVFIRKVYTILLIQLLVTLGVVALFTFCDPVKDYVQANPGW-------YWASYA--QKVRRVFIRKVYTILLIQLLVTLGVVALFTFCDPVKDYVQANPGW-------YWASYA--QKVRRVFIRKVYTILLIQLLVTLGVVALFTFCDPVKDYVQANPGW-------YWASYA--KNVRRVF IRKVY I I LMIQLSLTAGVIALFTFCDPVRSY IQARPGW-------YWASYA--KAIRRTFIRKVYAI LMVQLLVTVAIVGLFTFCTPVRFFIQTHPSM-------YMASYL--RNIRRVFIRKVYAI LMIQLLVTLAIVALFTFCDPVKDYIQSNPGW-------YWASYA--RNIRRVFIRKVYTI LMIQLLVTLAIVSLFTFCDPVKDY I SNPGW-------YWASYA--RNIRRVFIRKVYAI LMIQLLVTLAIVALFTFCDPVKDY IQTNPGW-------YWASYA--KNIRRMF IRKVFC I LMVQLMVTF SVVSLFTFCE PVRKFVQYNRVFYL---TSYMTFMG--RKVRHTFIRKVYSI ISVQLLITVAI IAIFTFVEPVSAFVRRN----------VAVYYVSYA RKVRHSFIQKVYCI ISVQLLITVAI IA IFTFVE PVGKYVRNN---------VAVYYVSYA RKVRHTFIQKVYCI ISVQLLITVAI IAVFTFVEPVSEYVRSN----------VAVYYVSYA RKVRHAFIRKVYTI ISIQLLVTVGI IAVFTFVKPVGDFVRAN---------MAVYYASYA RKVRHAFIRKVYTI IS IQLLVTVA I IAVFTFVKPVGDFVRAN---------LAIYYASYA RKVRHTFIRKVYTI IS IQLLITVAI IA I FTFVKPVGDFVRKN---------LYVYYVSYA RKVRHTFIRKVYTI ISIQLLITVAIIAIFTFVKPVGEFVRRN----------LYVYYVSYA RKVRHTFIRKVYTIISIQLLITVAI IAIFTFVKPVGDFVRRN----------LYVYYVSYA TAVRHAFIRKVYLILTAQLSVTVAIVAAFTFSESVQKFVQGNSALYWTAYGVFIVVYLV-LS IRHAFIRKVYLILASQLLVTTAIVAIFTFVEPVRLFVQRNQAVYW----ASYAVYA--LS IRHAFIRKVYLILASQLLVTTG IVA IFTFVHPVKTFVQENRAVYW----ASYAVYI--LS IRHAFIRKVYLI LAAQLLVTTAIVAVFT FVQPVRVFVQRNRAIYW----ASYGVYL--- 
bioRxiv preprint doi: https://doi.org/10.1101/2020.10.26.356402; this version posted October 27, 2020. The copyright holder for this preprint (which was not certified by peer review) is the author/funder, who has granted bioRxiv a license to display the preprint in perpetuity. It is made available under aCC-BY-NC-ND 4.0 International license.

LS IRHAFIRKVYLI LASQLLVTTAIVAVFTFVQPVRYFVQKNQAIYW----ASYAVYI--TDVRHSFIRKVYLILAAQLLVTAAVVAIFTFVEPVGLFVRKNPAIYW----VSYAIYF--PQYVKDRI HSTYMYLAGS IGLTALSAIAISRT PVLMNFMMRGSWVTI----G---VTFAA-PQYVKDRIHSTYMYLAGS IGLTALSALAVARTPALMNFMMTGSWVTI---G---ATFAA-PQYVKDRIHSTYMYLAGS IGLTALSALALARSPALMNFMMTGSWMTI---G---ATFAA-PQYVKDRIHSTYMYLAGS IGLTALSAVAVSRTPALMNFMMRGSWITI---G---ATFAA-PQYVKDRI HSTYMYLAGS IGLTALSAMAVSRTPALMNFMMRGSWITI---G---ATFAA-PQYVKDRIHSTYMYLAGS IGLTALSALAVSRT PVLMNFMMRGSWVTI---G---ATFAA-PQYVKDRIHSTYMYLAGSIGLTALSALAVSRTPVLMNFMMRGSWVTI---G---ATFAA-PQYVKDRIHSTYMYLAGS IGLTALSALAVSRTPVLMNFMMRGSWVTI---G---ATFAA-PQYVKDRI HSTYMYLAGS IGLTALSALAVSRTPVLMNFMMRGSWVTI---G---ATFAA-PQYVKERIRSTYMYFAGS IGLTALSAVAVSRS PMLMNLLMKNSFLAI---G---ATFAA-PQYVKDRIHSTYMYFAGSVGLTALSAVAVSRTPALLGMMMRGSWLAI---G---ATFAA-PQYVKDRIHSTYMYFAGS IGLTALSAVAVSRTPALMGLMMRGSWLAI---G---ATFAA-PQYVKDRIHSTYMYFAGSVGLTALSAVAVSRTPALMGLMMRGSWLAI---G---ATFAA-PQYVRDRIHSTYLYFAGSVGLTALSAVAISRTPVLMGIMMSNSWLAI---G---ATFAA-TPSTQQHLKKVYASFALCMFVAAAGAYVHMVTHFIQAGLLSALGSLI---L----MIW--TPSTQQHLKKVYASFALCMFVAAAGAYVHVVTHFIQAGLLSALGSLA---L----MIW--TPSTQQHLKKVYASFALCMFVAAAGAYVHVVTRFIQAGLLSALGALA---L----MIC--TPSTQQHLKKVYASFALCMFVAAAGAY IHVVTHFIQAGLLSALGSLG---L----MIW--TPSTQQHLKKVYASFALCMFVAAA GAYVHVVTHFIQAGLLSALGSLG---L----MIW--TPSTQQHLKKVYASFALCMFVAAAGAYVHVVTHFLQAGLLSALGSLG---L----MIW--TPSTQQHLKKVYASFALCMFVAAMGAYVHVVTHFLQAGLLSALGSLG---L----MIW--TPSTQQHLKKVYASFALCMFVAAMGAYVHVVTHFLQAGLLSALGSLG---L----MIW--TPSTQQHLKKVYASFALCMFVAAAGAYVHVVTHFLQAGLLSALGSLG---L----MIW--SPSTQQHLKKVYSSLAICMFVAAAGAYVHVVTRIFQGGLLSMLLSLG--------LMLW-SHSTQAHLKNVYSS LSVCMI LAAAGSYVHVVTRLFQGG I LSALGSLG---L----MFW--SHSTQVHLKNVYSSLAVCMFVAAAGSYVHVVTRLFQGGMLSVLASLG---M----MFW--SHSTQVHLKNVYSSLAVCMFVAAAGSYVHVVTRLFQGGLLSVLGSLG---M----MFW--SHSTQAHLKNVYASLAVCMFVAAAGAYVHVVTGLFQGGVLSVLGSLG---M----MFW---

IFALTL-------HRHKHPLNLYLLCGFTLSESLTLASVVTFYDV----HVVMQAFMLTT IFALTL-------HRHKHPLNLYLLCGFTLSESLTLASVVTFYDV----HVVMQAFMLTT IFALTL-------HRHKHPLNLYLLCGFTLSESLTLASVVTFYDV----HVVMQAFMLTT IFALTL-------HRHKHPLNLYLLCGFTLSESLTLASVVTFYDV----HVVMQAFMLTT IFALTL--------HRHKHPLNLYLLCGFTLSESLTLASVVTFYDV----HVVMQAFMLTT IFALTL-------HRHKHPLNLYLLCGFTLSESLTLASVVTFYDV----HVVMQAFMLTT IFALTL--------HRHKHPLNLYLLCGFTLSESLTLASVVT FYDV----HVVMQAFMLTT IFALTL-------HRHKHPLNLYLLCGFTLSESLTLASVVTFYDV----HVVMQAFMLTT IFALTL-------HRHKHPLNLYLLCGFTLSESLTLASVVTFYDV----HVVMQAFMLTT IFALTL-------HRHKHPLNLYLLCGFTLSESLTLASVVTFYDV----HVVMQAFMLTT IFALTL-------HRHKHPLNLYLLCGFTLSESLTLASVVTFYDV----HVVMQAFMLTT IFALTL------HRHKHPLNLYLLCGFTLSESLTLASVVTFYDV----HVVMQAFMLTT

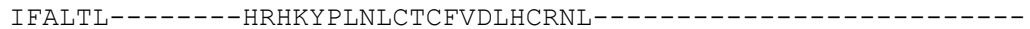
IFALTL--------HRHKHPLNLYLLCGFTLSESLTLASVVTFYDV----HVVMQAFMLTT IFALTL-------HRHKHPLNLYLLCGFTLSESLTLASVVTFYDV----HVVMQAFMLTT IFALTL--------HRHKHPLNLYLLCGFTLSESLTLASVVTFYDV----HVVMQAFMLTT IFALTL-------HRHKHPLNLYLLCGFTLSESLTLASVVTFYDV----HVVMQAFMLTT IFALTL-------HRHKHPLNLYLLCGFTLSESLTLASVVTFYDV----HVVMQAFMLTT IFALTL-------HRHEHPLNLY I LCGFTLLESLTLASVVTFYDA----RIVMQAFMLTT IFALTL-------HRHEHPLNLY ILCGFTLLESLTLASVVTFYDA----RIVMQAFMLTT IFALTL-------HRHKHPLNLYLLCGFTLLESLTLASVVTFYDA----RIVIQAFMLTT IFALTL-------HRHKHPLNLYLLCGFTLLESLTLASVVTFYDA----RIVIQAFMLTT IFALTL-------HRHKHPLNLYLLCGFTLLESLTLASVVTFYDA----RIVMQAFMLTT LMNVL-------VMYRHKRPVNSYLLVAFTFMEAVSVATTLTFYEY----STILQSLFLTC LFALFL-------NKQNFPANMY LFVGFTLLEAINVAFFAS FYNA----YTVIQAAFMT I LIFAL------ILNRHKYPLNLYLLFGFTLLEALTVAVVVTFYDV----YIILQAFILTT LIFAL-------TLHRHTHPLNLYLLFAFTLSESLAVAAVVT FYDV----YLVLQAFIMTT LIFAL------TLHRHTHPLNLYLLFAFTLSEALTVATVVTFYDG----HLVLHAFILTA LILAL------TVNRHKHP LNLYLLFGFTLLESLTVAFVVT FYDV----YVVLQAFILTC LILAL------TVNRHKHPLNLYLLFGFTLLESLTVAF IVTFYDV----YVVLQAFILTC LILAL-------TVNRHKHPLNLYLLFGFTLFEALTVAFVVTFYDV----YI I LQAFILTT LILAL------TVNRHKHPLNLYLLFGFTLFEALTVAFVVTFYDV----YI I LQAFILTT LILAL-------TVNRHKHPLNLYLLFGFTLFEALTVAFVVTFYDV----CI I LQAFILTT LVLAL------TVNRHKHPLNLYLLFGFTLFEALTVAFVVTFYDV----YIILQAFILTT LILAL-------TVNRHKHPLNLYLLFGFTLFEALTVAFVVTFYDV----YIILQAFILTT LI IAL-------AIYRHQHP INLYLLFAFTLFEA ITVATAVTFYQY----SVVLQAFVLTT LLLAL------AMYRHQHPANLYLLFAFTLLEAVSVATALTFYEY----STVLQALFLTC LLLAL------AVYRHQHPVNLYLLLVFTLLEAVSVATALTFYEY----STVLQALFLTC LLLFL------AIYRHQHPVNFYLLLVFTLLEAVSVATALTFYEY----STVLQALFLTC VFFISLIVLSCCGDFRRKHPWNLVALSVLTASLSYMVGMIASFYNT----EAVIMAVGITT 
bioRxiv preprint doi: https://doi.org/10.1101/2020.10.26.356402; this version posted October 27, 2020. The copyright holder for this preprint (which was not certified by peer review) is the author/funder, who has granted bioRxiv a license to display the preprint in perpetuity. It is made available under aCC-BY-NC-ND 4.0 International license.

IFFISLIVLSCCGDFRRKHPWNLVALS ILTVSLSYMVGMIASFYNT----EAVIMAVGITT IFFISLIVLSCCGDFRRKHPWNLVALS I LTISLSYMVGMIASFYNT----EAVIMAVGITT IFFVSLIVLSCCGDFRRKHPWNLVALS ILTVSLSYMVGMIASFYNT----EAVIMAVGITT VFFISLIVLSCCGDFRRKHPWNLVALSVLTVSLSYMVGMIASFYNT----EAVIMAVGITT VFFISLIVLSCCGDFRRKHPWNLVALS ILTVSLSYMVGMIASFYNT----EAVIMAVGITT VFFISLIVLSCCGDFRRKHPWNLVALS ILTVSLSYMVGMIASFYNT----EAVIMAVGITT VFFVSLIVLSCCGDFRRKHPWNLVALS I LTVSLSYMVGMIAS FYNT----EAVIMAVGITT VFFISLIVLSCCGDFRRKHPWNLIALS I LTVSLSYMVGMIASFYNT----EAVIMAVGITT VFFVTLIALSCCGQIRRKHPWNLIALS I LTLSMSYMAGMIASFYST----DSVVMAVGITA VFFVSLIVLSCCGDFRRKHPWNLVALS ILTLSLSYMVGMIASFYNT----DTVIIAVGITA VFFVSLIVLSCCGDFRRKHPWNLVALS I LTLSLSYMVGMIASFYNT----ETVIMAVGITA VFFVSLIVLSCCGDFRRKHPWNLVALS ILTLSLSYMVGMIASFYDT----ETVIMAVGITA VFFATYLTLACCSGPRRHFPWNLILLTVFTLSMAYLTGMLSSYYNT----TSVLLCLGITA VFFATYLTLACCSGPRRHFPWNLILLTIFTLSMAYLTGMLSSYYNT----TSVLLCLVITA VFFATYLTLACCSGPRRHFPWNLILLTIFTLSMAYLTGMLSSYYNT----TSVLLCLGITA VFFATYLTLACCSGPRRHFPWNLILLTIFTLSMAYLTGMLSSYYNT----TSVLLCLSITA VFFATYLTLACCSGPRRHFPWNLILLTIFTLSMAYLTGMLSSYYNT----TSVLLCLSITA VFFATYLTLACCSGPRRHFPWNLILLTIFTLSMAYLTGMLSSYYNT----TSVLLCLGITA VFFATYLTLACCSGPRRHFPWNLILLTIFTLSMAYLTGMLSSYYNT----TSVLLCLGITA VFFATYLTLACCSGPRRHFPWNLILLTIFTLSMAYLTGMLSSYYNT----TSVLLCLGITA VFFATYLTLACCSGPRRHFPWNLILLTIFTLSMAYLTGMLSSYYNT----TSVLLCLGITA VFFVTYMTLACCPGPRRYFPWNLILLGIFTLAMSYMTGMMS SFYNT----KSVLLCFTITA MFFATYIALSCCGELRRQFPWNITLLVIFTLSMAFMMGFVSSFYNT----KSVVLCLGITA VFFVTYLTLSCCSAPRRQFPWNLILLAIFTLSLSYMTGMLSSFYNT----KSVVMCLGITV VFFVTYLTLSCCSAPRRQFPWNLILLGIFTLSLSYMTGMLSSFYNT----KSVVMCLGITV VFFVTYLTLSCCSTPRRQFPWNLILLAIFTLSLSYMTGMLSSFYNT----KSVVICLGITA TYLM----LVCSTNARRRYPTNMILLA IFTLAMSYMAGMLASYHNT----KVVMLSVGITA VFVVTYLI LACCQGPRRRFPWNI I LLTLFTFAMGFMTGT IS SMYQT----KAVI IAMI ITA VFLVTYLTLACCQGPRRRFPWNI I LLT IFTLALGFVTGT ISSMYET----KAVI IAMI ITA VFIVTYLILVCCQGPRRRFPWNI I LLTIFTLALGFMTGAISSMYET----KAVI IAMIITA VFLVTYLTLACCQGPRRRFPWNI I LLI I FTLAMAYMTGT ISSVYKT----KAVI IAMI ITA VFLATYLTLICCQGPRRRFPWNI I LLILLTLAMAYMTGT ISSVYKT----KAVI IAMIITA VFLATYLTLACCQGPRRRFPWNI I LLTIFTLAMGFMTGT ISSVYET----KAVI IAMI ITA VFLATYLTLACCQGPRRRFPWNI I LLTIFTLAMGFMTGT ISSVYET----KAVI IAMI ITA VFLATYLTLACCQGPRRRFPWNI ILLTIFTLAMGFMTGT ISSVYET----KVVI IAMI ITA -------LICCEGPRRRFPWNVILLAIFTLAMSYLTGTIASYYST----KSVFMCLGITT ---VTH IVLVCCKGPRRKFPWNI I LLF I FTLALSYMSGCISSFYDT----KAVFLALGVTG ---VTHLVLVCCKGPRRKFPWNV ILLLIFTLALSYMTGAISSYYST----KAVFLALGITA ---VTHLVLVCCKGPRRKFPWNLI LLS I FTLAMSYMTGS IS SYYDT----KAVFLAMGITA ---VTHLVLVCCKGPRRKFPWNVILLFLFTLALSYMTGS ISSYYDT----KAVFLALGITA ---VTH IVLVCCQGPRRRF PWNLLLLA I FTLALSFMTGNIASYYST----RAVFLALAITV --MVGA GMLVRS IPYDQS PGPKHLAWLLHSGVMGAVVAPLTILGGP----LLIRAAWYTAG --MIGA GMLVHS ISYEQS PGPKHLAWMLHSGVMGAVVAPLTI LGGP----LLLRAAWYTAG --MIGAGMLVQS ISYEQSPGPKHLAWMLHSGVMGAVVAPLTILGGP----LLLRAAWYTAG --MIGAGMLVQS ISYEQSPGPKHLAWLLHSGVMGAVVAPLTI LGGP----LLLRAAWYTAG --MIGAGMLVQS ISYEQSPGPKHLAWLLHSGVMGAVVAPLT I LGGP----LLVRAAWYTAG --MIGAGMMVQS I PYDQS PGPKHLAWLLHSGVMGAVVAPLT I LGGP----LLVRAAWYTAG --MIGAGMMVQS I PYDQSPVPKHLAWLLHSGVLGAVVAPLTVLGGP----LLIRAAWYTAG --MIGAGMMVQS I PYDQSPVPKHLAWLLHSGVLGAAVAPLTVLGGP----LLIRAAWYTAG --MIGAGMMVRS I PYDQSPVPKHVAWLLHSGVLGAVVAPLTMLGGP----LLIRAAWYTAG --MIGAGMLVRS LPYTERP SMKHLAWLLHAGVMGAVVAPVTLLGGP----LLIRAAWYTAG --MI GA GMLVRS ISYEHSPVPKHLAWMLHAGVMGAVIAPLTLLGGP----LMIRAAWYTAG --MIGAGMLVRS ISYEQS PMPKHLAWMFHAGVMGAVIAPLTLLGGP----LVMRAAWYTAG --MIGA GMLVRS ISYEHS PLPKHLAWMLHAGVMGAVIAPLTLLGGP----LMMRAAWYTAG --LIGAGVLVRSVSYEHSPVAKHLSWALYAGVFGAVVAPLTLLGGP----LMLRAVWCTAG LMAT-------PHSHETEQKRLGLLAGFAFLTGVGLGPALEFCIAVNPS I LPTAFMGTAM LMAT-------PHSHETEQKRLGLLAGFAFLTGVGLGPALELCIAVNPS I LPTAFMGTAM LMAT-------PHSHETEQKRLGLLAGFAFLTGVGLGPALELCIAINPS I LPTAFMGTAM LMAT-------PHSHETEQKRLGLLAGFAFLTGVGLGPALDLCIAINPS I LPTAFMGTAM LMAT-------PHSHETEQKRLGLLAGFAFLTGVGLGPALDLCIAINPS I LPTAFMGTAM LMTV-------PHSHETEQKRLGLLAGFAFLTGVGLGPALELCIAINPS I LPTAFMGTAM LMTT-------PHSHETEQKRLGLLAGFAFLTGVGLGPALELCIAINPS I LPTAFMGTAM LMTT-------PHSHETEQKRLGLLAGFAFLTGVGLGPALELCIAINPS I LPTAFMGTAM LMTT-------PHSHETEQKRLGLLAGFAFLTGVGLGPALELCIAINPS I LPTAFMGTAM LSFT-------PHNSETESKRLLILAGFAFFTGTGLGP IMDFVISVDPS I IPTSFLATAL LAMT-------PHNPETEKKRLAI LAGFAFLTGLGLGPTLDFVIAVNPS I IATAFMGTSV LAMT-------PHNSKTEKKRLA I LAGFAFLTGVGLGPTLDFVIAVNPS I IVTAFMGTSV LAMT-------PHNPETEKKRLA I LAGFAFFTGVGLGPTLDFVIAVNPS I IVTAFMGTSV LAMT-------PHNPETEKKRLGMLAVFAFLTGVGLGPTLDFVIAVNPS I IMTAFMGTSM 
bioRxiv preprint doi: https://doi.org/10.1101/2020.10.26.356402; this version posted October 27, 2020. The copyright holder for this preprint (which was not certified by peer review) is the author/funder, who has granted bioRxiv a license to display the preprint in perpetuity. It is made available under aCC-BY-NC-ND 4.0 International license.

AVFLALTAYTLQSKRDFSKLGAGLFVTLWILILSGLLRI----F-VQSETVELVLSAFGAL AVFLALTAYTLQSKRDFSKLGAGLFVTLWILILSGLLRI----F-VQSETVELVLSAFGAL AVFLALTAYTLQSKRDFSKLGAGLFVTLWILILSGLLRI----F-VQSETVELVLSAFGAL AVFLALTTYTLQSKRDFSKLGAGLFATLWILILSGLLRI----F-VQNETVELVLSALGPL AVFLALTTYTLQSKRDFSKLGAGLFATLWILILSGLLRI----F-VQNETVELVLSALGPL AAFLALTTYTLQSKRDFSKLGAGLFAALWILILSGLLRI----F-VQNETVELVMSAFGAL AAFLALTTYTLQSKRDFSKLGAGLFAALWILILSGLLRI----F-VQNETVELVLSAFGAL AAFLALTTYTLQSKRDFSKLGAGLFAALWILILSGLLRI----F-VQNETVELALSAFGAL AAFLALTTYTLQSKRDFSKLGAGLFAALWILILSGLLRI----F-VQNETVELVMSAFGAL AAFLALTTYTLQSKRDFSKLGAGLFAALWILILSGLLGI----F-VQNETVELVLSAFGAL AVFLALTTYTLQSKRDFSKLGAGLFAALWILILSGLLRI----F-VQNETVELVLSAFGAL AVETA AVFLALTTYTLQSKRDFSKLGAGLFATLWILILSGLLRI----F-VQNETVELVLSAFGAL AVFLALTTYTLQSKRDFSKLGAGLFATLWILILSGLLRI----F-VQNETVELVLSAFGAL AVFLALTTYTLQSKRDFSKLGAGLFATLWILILSGLLRI----F-VQNETVELVLSAFGAL AVFLALTTYTLQSKRDFSKLGAGLFATLWILILSGLLRI----F-VQNETVELVLSAFGAL AVFLALTTYTLQSKRDFSKLVTGLFAAFWILILSGVLRI----K-FKIELIKNI------AVFLALTTYTLQSKRDFSKLVTGLFAAFWILILSGVLRI----K-FKIELIKNI------AVFLALTTCTLQSKRDFSKLVTGLFAAFWILILSGVLRI----K-FKIELIKNI------AVFLALTTCTLQSKRDFSKLVTGLFADFWILILSGVLRI----K-FKIELIKNI------AVFLALTTCTLQSKRDFSKLVTGLFAAFWILILSGVLRI----K-FKIELIKNI------AVFAGLTVYTFQSKKDFSKLGAGLFACILIFVIAMLIQL----F-YNSDSADLVISGFGAV VAFTGLTVYTFQSTRNFSKLGAYLFSCLCVLCVGCILSMV---F--YNEILEIVLAAFGCF TVFFGLTVYTLQSKKDFSKFGAGLFALLWI LCLSGFLKF----F-FYSEIMELVLAAAGAL AVFLGLTAYTLQSKRDFTKFGAGLFAGLWILCLAGFLKL----F-FYSETMELVLASLGAL AVFLGLTAYTLQSKRDFSKFGAGLFACLWILCLAGFLKV----F-FYSQTVELVLASLGAL AVFLGLTVYTLQSKRDFSKFGAGLFAGLWILCLSGILRL----F-FYSETVELVLAAGGAL AVFLGLTVYTLQSKRDFSKFGAGLFAGLWILCLSGILRL----F-FYSETVELVLAAGGAL AVFLGLTGYTLQSKRDFSKFGAGLFAVLWILCLSGILKL----F-FYSQTMELVLAAVGAL AVFLGLTVYTLQSKRDFSKFGAGLFAVLWILCLSGILKL----F-FYSQTMELVLAAVGAL AVFLGLTVYTLQSKRDFSKFGAGLFAVLWILCLSGILKL----F-FYSQTMELVLAAVGAL AVFLGLTVYTLQSKRDFSKFGAGLFAVLWI LCLSGILKL----F-FYSQTMELI LAAVGAL AVFLGLTVYTLQSKRDFSKFGAGLFAVLWILCLSGILKL----F-FYSQTMELVLAAVGAL AVFLGLTSYTFQSKRDFSKYGAGLFACLWILILAGFFRL----F-FFSETMELVFASAGAL AVFAGLTAYTFQSKRDFSKLGAGLFACLWILIIASFMRF----F-FNSDSTELVFAGAGAL AVFAGLTVYTLQSKRDFSKMGAGLFACLWILLIASFMRL----F-FNSDGTELVMAGAGAL AVFVGLTAYTFQSKRDFSKMGAGLFACLWILVIAS FMRF----F-FNNDSTEVVLAGAGAL AVCFTVVIFSMQTRYDFTSCMGVLLVSMVVLFIFAILCI----F-IRNRILEIVYASLGAL AVCFTVVIFSMQTRYDFTSCMGVLLVSVVVLFIFAILCI----F-IRNRI LEIVYASLGAL AVCFTVVIFSMQTRYDFTSCMGVLLVSVVVLFIFAILCI----F-IRNRI LEIVYASLGAL TVCFTVVIFSMQTRYDFTSCVGVLLVSVVVLILFAILCI----F-IRSRVLEIVYASLGAL TVCFTVVIFSMQTRYDFTSCMGVLLVSLVVLVIFAILCI----F-IRNRI LEIVYASLGAL TVCFTVVIFSMQTRYDFTSCMGVLLVSLVVLVIFAILCI----F-IRNRILEIVYASLGAL TVCFTVVIFSMQTRYDFTSCMGVLLVSLVVLVIFAILCI----F-IRNRI LEIVYASLGAL TVCFTVVIFSMQTRYDFTSCVGVLLVSVVVLILFAILCI----F-IRSRVLEIVYASLGAL TVCFTVVIFSMQTRYDFTSCMGVLLVSLVVLVVFAVLCI----F-IRNRI LEIVYASLGAL AVCFTVVIFSMQTKYDFTSCMGVMLVSVVVLFFFG I LCI----F-IQNKI LQIVYASVGAL VVCFTVVLFSLQSKYDFTSCRGVLFVCLIVLLLFS ILCI----F-IRDKI LHIVYASLGAL VVCFTVVLFSLQSKYDFTSCRGVLFVCLIVLLLFSFLCI----F-IRHKILHIVYASLGAL VVCFTVVLFSLQSKYDFTSCRGVLFVCLIVLLLFS ILCI----F-IRHRI LHIVYASLGAL LVCLSVTVFSFQTKFDFTSCQGVLFVLLMTLFFSGLILAILLPF-QYVPWLHAVYAALGAG LVCLSVTIFSFQTKFDFTSCQGVLFVLLMTLFFSGLLLAVLLPF-QYVPWLHAVYAVLGAG LVCLSVTIFSFQTKFDFTSCHGVLFVLLMTLFFSGLLLAILLPF-QYVPWLHAVYAVLGAG LVCLSVTVFSFQTKFDFTSCQGVLFVLLMTLFFSGLILAILLPF-QYVPWLHAVYAVLGAG LVCLSVTVFSFQTKFDFTSCQGVLFVLLMTLFFSGLILAILLPF-QYVPWLHAVYAVLGAG LVCLSVTVFSFQTKFDFTSCQGVIFVLLMTLFFSGLILAILLPF-QYVPWLHAVYAVLGAG LVCLSVTVFSFQTKFDFTSCQGVIFVLLMTLFFSGLILAILLPF-QYVPWLHAVYAVLGAG LVCLSVTVFSFQTKFDFTSCQGVIFVLLMTLFFSGLILAILLPF-QYVPWLHAVYAVLGAG LVCLSVTVFSFQTKFDFTSCQGVIFVLLMTLFFSGLILAILLPF-QYVPWLHAVYAVLGAG LVCLAVTIFSFQTKFDFTSCHGVLFVLLIVLCISGLVLAIVLPF-QYVPWLHAVYAVLGA I LVCLSVTVFSFQSKVDVTSCQGI LFS LCMVMLLCAITLS IVVPF-GYVPWLHAIYAVLGAI AVCLLVTIFSFQTKFDVTSYQGVLFVFCMVMF I SGLVLALVLPF-QYVPWLDAIYAALGAI AVCLLVTVFSFQTKFDVTSYQGVLFVFCMVMFVSGLVLALVLPF-QYVPWLDATYAVLGAI AVCLLVTVFSFQTKFDVTSYQGVLFVFCTVMF ISGLVLAF I LPF-QYVPWLDGIFAALGAI LVCLAITLFCFQSRVDFTTCHGLLFS LMMVLMITGLLLFFTAPF-GYIPWLHTAYAGFGAL VVS ISVTI FCFQTKVDFTSCTGLFCVLGIVLLVTG IVTS IVLYF-QYVYWLHMLYAALGA I VVS ISVTIFCFQTKVDFTSCTGLFCVLGIVLMVTGIVTS IVLIF-KY IYWLHMVYAALGA I VVS ISVTIFCFQTKVDFTSCTGLICVLGIVLAVTGAVTSVVLFF-EY I YWLHMVYAGLGAI VVSISVTIFCFQTKVDFTSCTGLFCVLGIVMTVTGIITVIVLVF-KYVYWLHMVYAALGAI VVSISVTIFCFQTKVDFTSCTGLFCVLAIVMVVTGI ITAIVLAF-KYVYWLHMVYAAVGAI 
bioRxiv preprint doi: https://doi.org/10.1101/2020.10.26.356402; this version posted October 27, 2020. The copyright holder for this preprint (which was not certified by peer review) is the author/funder, who has granted bioRxiv a license to display the preprint in perpetuity. It is made available under aCC-BY-NC-ND 4.0 International license.

VVSISVTIFCFQTKVDFTSCTGLFCVLGIVMMVTGIVTAIVLSF-KYIYWLHMVYAALGA I VVSISVTIFCFQTKVDFTSCTGLFCVLGIVMMVTGIVTAIVLSF-KYIYWLHMVYAALGAI VVS ISVTIFCFQTKVDFTSCTGLFCVLGIVMMVTGIVTAIVLSF-KYIYWLHMVYAALGA I LVCLGVTLFCFQTKVDFTSCTGLFCALGIGLFVGGIVISIVLSF-KYIPWLHSLYAAAAAV VVCVAVTVFCFQTKVDFTKCQGLFCVLGIVIFVLGIITAIVLSF-KYIPWLHMLYAALGAI VVCIAVTVFCFQTKVDFTKCQGLFCVLGIVIFVTGIITTIVLSF-KYILWLHMLYAALGAI VVCIAVTVFCFQTKVDFTKCQGLFCVLGIVMFVTGIITCIVLSE-KYIPWLHMLYAAMGAI VVCIAVTVFCFQTKVDFTKCQGLFCVLGIVMLLTGI IAT IVLSF-KY I LWLHMLYAAMGA I VVCVAVTVFCFQTKVDFTKCSGFFCVLGIVVFVTGI ITAIVLSF-KYVPWLHMLYAS IGAI IVGGLSTVAMCAPSEKFLNMGAPLGVGLGLVFVSSLGSMFLPPTTVAGATLYSVAMYGGLV IVGGLSTVAMCAPSEKFLNMGAPLGVGLGLVFASSLGSMFLPPTSVAGATLYSVAMYGGLV IVGGLSTVAMCAPSEKFLNMGAPLGVGLGLVEASSLGSMFLPPTSVAGATLYSVAMYGGLV IVGGLSTVAMCAPSEKFLNMGAPLGVGLGVVFVSSLGSMFLPPTTVAGATLYSVAVYGGLV IVGGLSTVAMCAPSEKFLNMGAPLGVGLGVVFVSSLGSMFLPPTTVAGATLYSVAVYGGLV IVGGLSTVAMCAPSEKFLNMGAPLGVGLGLVFVSSLGSMFLPPTTVAGATLYSVAIYGGLV IVGGLSAVAVCAPSEKFLNMGAPLGVGLGLVFVSSLGSMFLPPTTVAGATLYSVAIYGGLV IVGGLSAVAVCAPSEKFLNMGAPLGVGLGLVFVSSLGSMFLPPTTVAGATLYSVAIYGGLV IVGGLSAVAMCAPSEKFLNMGAPLGVGLGLVFVSSLGSMFLPPTTVAGATLYSVAIYGGLV IVGGLSTVAMCAPSEKFLNIGGPLAIGLGFVFASSVGSMFLPPTSMLGAGLYSVAIYGGLV IVGGLSTVAMCAPSEKFLNMGGPLAVGFGVVFASSLGSMFLPPTSAFGAGLYSVAIYGGLV IVGGLSTVAMCAPSEKFLNMGGPLAVGFGVVFASS IGSMFLPPTSAFGAGLYSVAIYGGLV IVGGLSTVAVCAPSEKFLNMGGPLAVGFGVVFASS IGSMFLPPTSAFGAGLYSVAVYGGLV VVGGLSTVAVCAPSERFLNMGGPLAVGLGLVLASTLGSMFLPPSTAVGAGLYSVSVYGGLL IFTCFTLSALYARRRSYLFLGGI LMSALSLLLLSS LGNV----F-FGS IWLFQANLYVGLV IFTCFSLSALYARRRSY LFLGG I LMSAMS LMLLSS LGNL----F-FGS IWLFQANLYLGLL IFTCFSLSALYARRRSYLFLGGI LMSAMS LMFVSSLGNL----F-FGS IWLFQANLYMGLL IFTCFTLSALYARRRSYLFLGGI LMSAMS LMLLSS LGNL----F-FGSVWLFQANLYMGLV IFTCFTLSALYARRRSYLFLGGI LMSAMS LMLLSSLGNL----F-FGSVWLFQANLYMGLV IFTCFTLSALYARRRSY LFLGGVLMSAMS LMLLSS LGNL----F-FGS IWLFQANLYVGLV IFTCFTLSALYARRRSYLFLGGVLMSAMS LMLLSS LGNL----F-FGS IWLFQANLYVGLV IFTCFTLSALYARRRSYLFLGGVLMSAMS LMLLSS LGNL----F-FGS IWLFQANLYVGLV IFTCFTLSALYARRRSYLFLGGVLMSAMS LMLLSS LGNL----F-FGS IWLFQANLYVGLV IFSCFTLSALYAQRRSYLFLGGILMSCLTVLCFLPLINLL---F--GSMLLFKVNGRRTFL IF ICFSLSALYAKRRSYLFLGGTLMSGLSFLFLLSVMNI----F-FGSLMLFKAHMYLGLL IFICFTLSALYAKRRSYLFLGGTLMSGLSLLFLMSVMNM----F-FGSVMVFKAHMYLGLL IF ICFTLSALYAKRRSY LFLGGTLMSGLS I LFLMS LMNM----F-FGS LMLFKAHMYLGLL IFICFS LSALYAKRRSYLFLGGTLMSGLSLLFLMSVMNM----F-FGSVMLFKAHMYLGLL

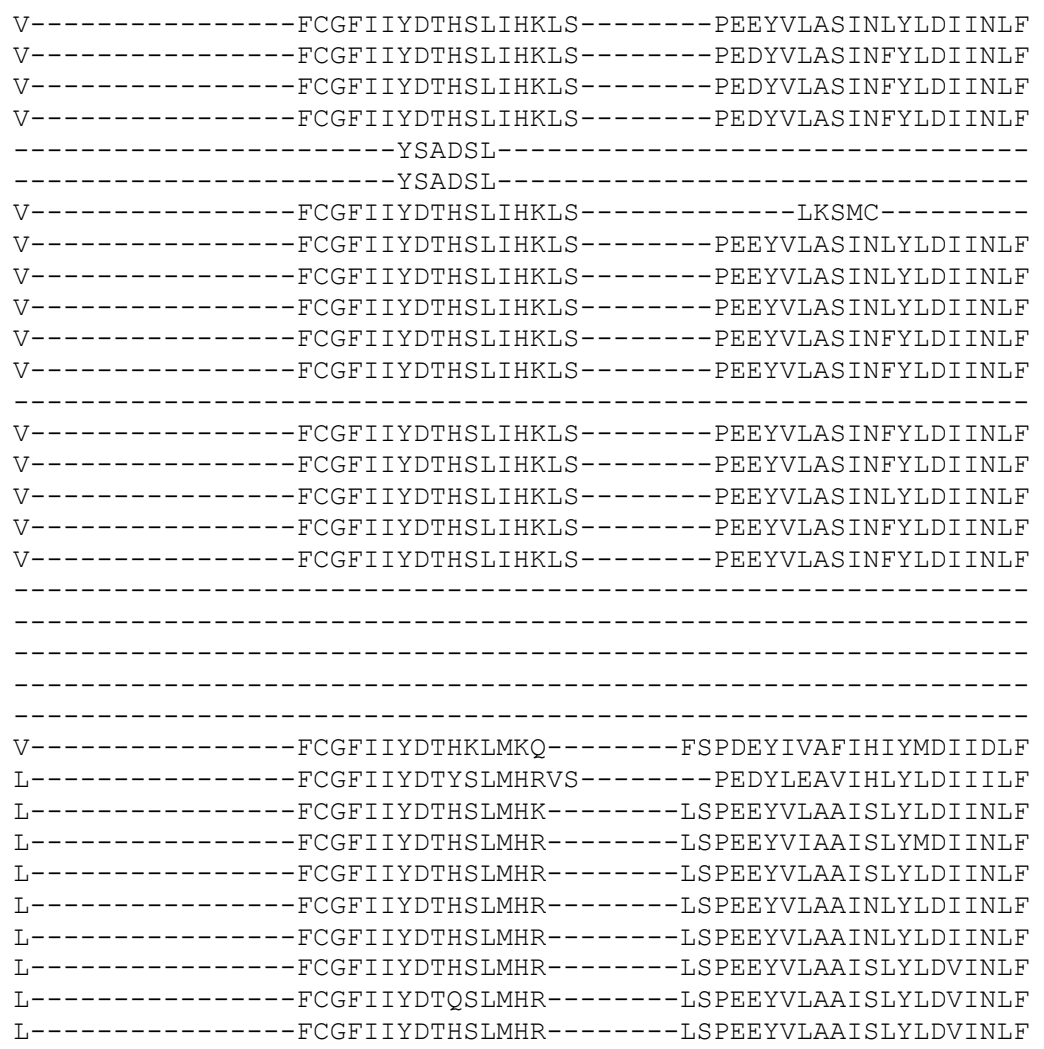


bioRxiv preprint doi: https://doi.org/10.1101/2020.10.26.356402; this version posted October 27, 2020. The copyright holder for this preprint (which was not certified by peer review) is the author/funder, who has granted bioRxiv a license to display the preprint in perpetuity. It is made available under aCC-BY-NC-ND 4.0 International license.

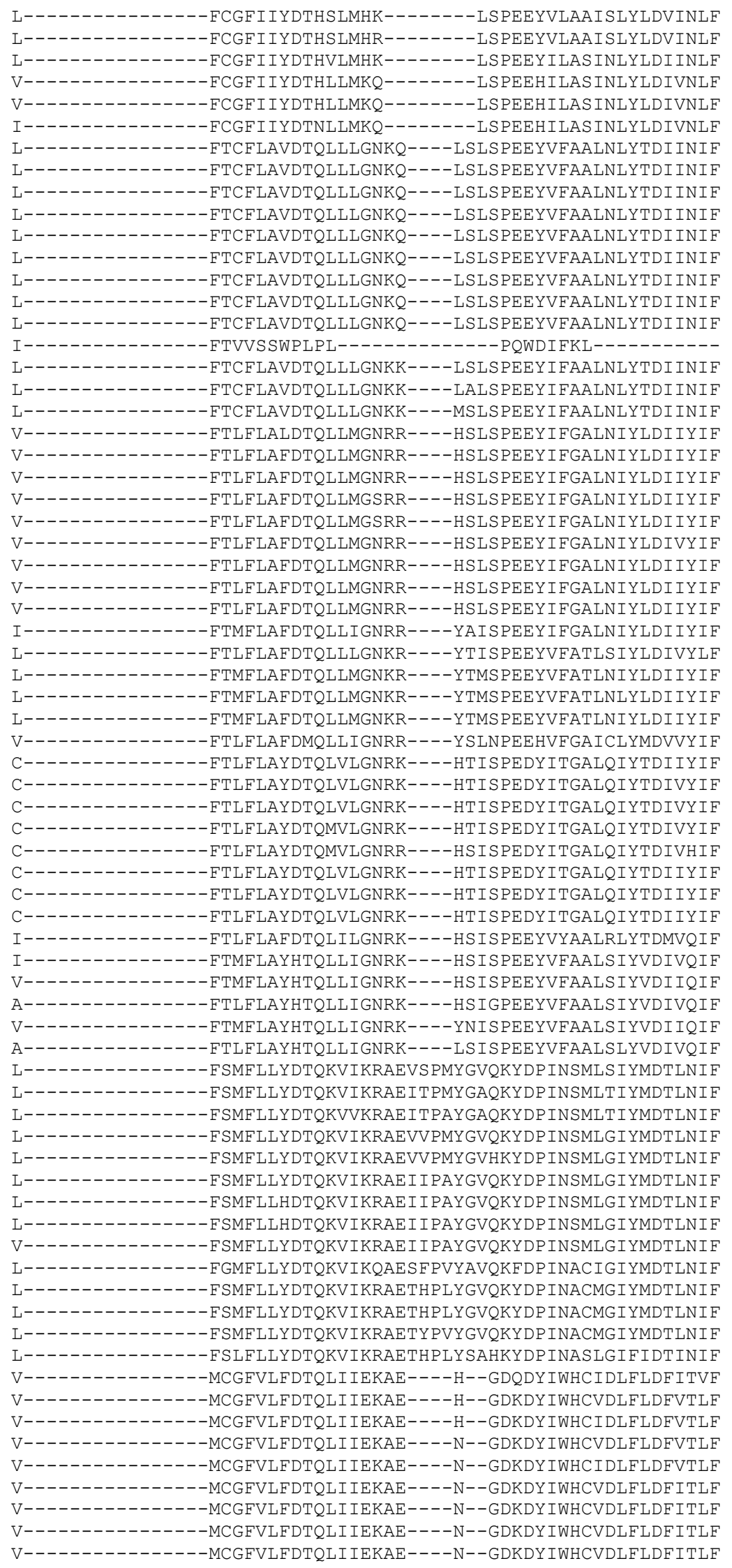


bioRxiv preprint doi: https://doi.org/10.1101/2020.10.26.356402; this version posted October 27, 2020. The copyright holder for this preprint (which was not certified by peer review) is the author/funder, who has granted bioRxiv a license to display the preprint in perpetuity. It is made available under aCC-BY-NC-ND 4.0 International license.

TPPPPKKCPAVPCACFPFCCSLTNN------------------------------
V--------------MCGFVLFDTQLIIEKAE----N--GDKDYVWHCVDLFLDFITIF
I--------------MCGFVLFDTQLIIEKAE----N--GDKDYIWHCVDLFLDFITIF
I--------------MCGFVLFDTQLIIEKAE----N--GDKDYVWHCVDLFLDFVTIF
I---------------MCGFVLFDTQLIIEKAE----N--GDKDYVWHCVDLFLDFITIF

LHLLQLLEVSNKK------

LHLLQLLEVSNKK------

LHLLQLLEVSNKK------

LHLLQLLEVSNKK------

------- SMTRTH------

-------SMTRTH------

$------------------1$

LHLLQLLEVSNKK------

LHLLQLLEVSNKK------

LHLLQLLEVSNKK------

LHLLQLLEVSNRK------

LHLLQLLEVSNKK------

I HIT

LHLLQLLEVSNKK------

LHLLQLLEVSNKK------

LHLLQLLEVSNKK------

LHLLQLLEVSNKK------

------------------

$------------------1$

------------------

------------------

MRILHILKSIKNO------

MDFLRILGESKSESKSITV

LHLLRFLEAVNKK------

LHLLKFLEAVNKK------

LHLLKFLDAVNKK------

LHLLRVLEAANKK------

LHLLRVLEAVNKK------

LHLLRFLEAVQKK------

LHLLRFLEAVNKK------

LHLLRFLEAVNKK------

LHLLRELEAVNKK------

LHLLRFLEAVNKK------

LHILRILESINKK------

LHILRVLDSMKKH------

LHILRLLDSMKKH------

LHILRLLDSMKKH------

LYILTIIGRAKE-------

LYILTIIGRAKE-------

LYILTIIGRAKE-------

LYILTIIGRAKE-------

LYILTIIGRAKE-------

LYILTIIGRAKE-------

LYILTIIGRAKE-------

LYILTIIGRAKE-------

LYILTIIGRAKE-------

$------------------$

LYILAIVGRSRE-------

LYILAIVGRSRE-------

LYILTIVGRSRE-------

TFFLQLFGTNRE------

TFFLQLFGTNRE-------

TFFLQLFGTNRE-------

TFFLQLFGTNRE-------

TFFLQLFGTNRE-------

TFELQLFGTNRE-------

TFFLQLFGTNRE-------

TFFLQLFGTNRE-------

TFFLQLFGTNRE-------

TFMLQIFGSNRE-------

SFLLQLTGQGRD-------

SFFLQIFGTKQE-------

SFFLQIFGTKQE------- 
bioRxiv preprint doi: https://doi.org/10.1101/2020.10.26.356402; this version posted October 27, 2020. The copyright holder for this preprint (which was not certified by peer review) is the author/funder, who has granted bioRxiv a license to display the preprint in perpetuity. It is made available under aCC-BY-NC-ND 4.0 International license.

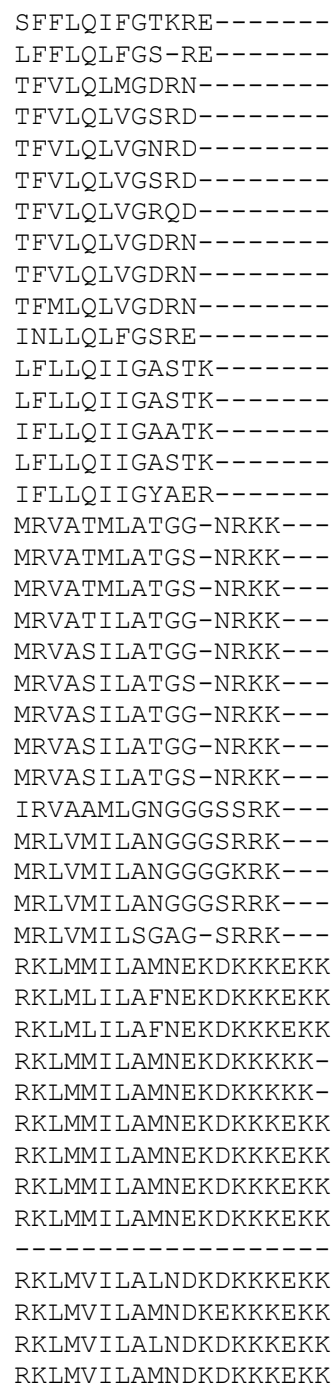

\section{Table S2.}

Phylip alignment for sequences used in phylogenetic analysis (Fig. 1B) 


\section{Supplementary sequences:}

piggybac SLP-mCherry-K3L:

actcttcctttttcaatattattgaagcatttatcagggttattgtctcatgagcggatacatatttgaatgtatttagaaaaataaacaaataggggttccgcgeacatttccccga aaagtgccacctaaattgtaagcgttaatattttgttaaaattcgcgttaaattttgttaaatcagctcattttttaaccaataggccgaaatcggcaaaatcccttataaatcaaa agaatagaccgagatagggttgagtgttgttccagtttggaacaagagtccactattaaagaacgtggactccaacgtcaaagggcgaaaaccgtctatcagggcgatg gcccactacgtgaaccatcacc ctaatcaagtttttggggtcgaggtgccgtaaagcactaaatcggaaccctaaagggagccccegatttagagcttgacggggaaag ccggcgaacgtggcgagaaaggaagggaagaaagcgaaaggagcgggcgetagggcgetggcaagtgtagcggtcacgctgcgcgtaaccaccacacccgccg cgcttaatgcgccgctacagggcgcgtccattcgccattcaggctgcgcaactgttgggaagggcgatcggtgcgggcetcttcgctattacgccagctggcgaaagg gggatgtgctgcaaggcgattaagttgggtaacgccagggttttcccagtcacgacgttgtaaaacgacggccagtgagcgcgectcgttcattcacgttttgaacccgt ggaggacgggcagactcgcggtgcaaatgtgttttacagcgtgatggagcagatgaagatgctcgacacgctgcagaacacgcagctagattaaccctagaaagataa tcatattgtgacgtacgttaaagataatcatgcgtaaaattgacgcatgtgttttatcggtctgtatatcgaggtttatttattaatttgaatagatattaagttttattatatttacactt acatactaataataaattcaacaaacaatttatttatgtttatttatttattaaaaaaaacaaaaactcaaaatttcttctataaagtaacaaaacttttatgagggacagcccccc

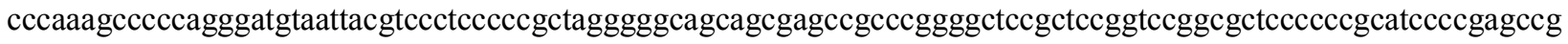
gcagcgtgcggggacagcccgggcacggggaaggtggcacgggatcgctttcctctgaacgcttctcgctgctctttgagcctgcagacacctggggggatacgggg aaaGGCCTCCACGGCCAGACTAGAACTTTTAAAAGAAAAGGGGGGATTGGGGGGTACAGTGCAGGGG AAAGAATAGTAGACATAATAGCAACAGACATACAAACTAAAGAATTACAAAAACAAATTACAAAAAT TCAAAATTTTATCGATCACGAGACTAGCCTCGAGGGATCatccggtgccegtcagtgggcagagcgcacatcgcccacagtcccc gagaagttggggggaggggtcggcaattgaacgggtgcctagagaaggtggcgcggggtaaactgggaaagtgatgtcgtgtactggctccgccttttcccgagggt gggggagaaccgtatataagtgcagtagtcgccgtgaacgttctttttcgcaacgggtttgccgccagaacacagctgaagcttcgaggggetcgcatctctccttcacgc

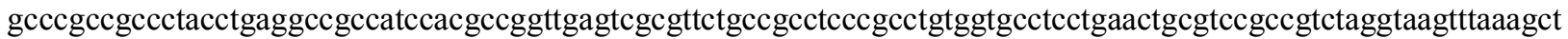
caggtcgagaccgggectttgtceggegetccettggagcetacctagactcagccggetctccacgetttgectgaccetgettgctcaactctacgtctttgtttcgttttct gttctgcgecgttacagatccaagctgtgaccggcgecGAATTCCTTCAGGGTGAGTTTGGGGACCCTTGATTGTTCTTTCTTT TTCGCTATTGTAAAATTCATGTTATATGGAGGGGGCAAAGTTTTCAGGGTGTTGTTTAGAATGGGAAG ATGTCCCTTGTATCACCATGGACCCTCATGATAATTTTGTTTCTTTCACTTTCTACTCTGTTGACAACCA TTGTCTCCTCTTATTTTCTTTTCATTTTCTGTAACTTTTTCGTTAAACTTTAGCTTGCATTTGTAACGAAT TTTTAAATTCACTTTTGTTTATTTGTCAGATTGTAAGTACTTTCTCTAATCACTTTTTTTTCAAGGCAATC AGGGTATATTATATTGTACTTCAGCACAGTTTTAGAGAACAATTGTTATAATTAAATGATAAGGTAGA ATATTTCTGCATATAAATTCTGGCTGGCGTGGAAATATTCTTATTGGTAGAAACAACTACATCCTGGTC ATCATCCTGCCTTTCTCTTTATGGTTACAATGATATACACTGTTTGAGATGAGGATAAAATACTCTGAG TCCAAACCGGGCCCCTCTGCTAACCATGTTCATGCCTTCTTCTTTTTCCTACAGCTCCTGGGCAACGGT ACCGGATCCCTGCAGAAGCTTCTAAAAATTGAAATTTTATTTTTTTTTTTTGGAATATAAATGGTGAGC AAGGGCGAGGAGGATAACATGGCCATCATCAAGGAGTTCATGCGCTTCAAGGTGCACATGGAGGGCT CCGTGAACGGCCACGAGTTCGAGATCGAGGGCGAGGGCGAGGGCCGCCCCTACGAGGGCACCCAGAC CGCCAAGCTGAAGGTGACCAAGGGTGGCCCCCTGCCCTTCGCCTGGGACATCCTGTCCCCTCAGTTCA TGTACGGCTCCAAGGCCTACGTGAAGCACCCCGCCGACATCCCCGACTACTTGAAGCTGTCCTTCCCC GAGGGCTTCAAGTGGGAGCGCGTGATGAACTTCGAGGACGGCGGCGTGGTGACCGTGACCCAGGACT CCTCCCTGCAGGACGGCGAGTTCATCTACAAGGTGAAGCTGCGCGGCACCAACTTCCCCTCCGACGGC CCCGTAATGCAGAAGAAGACCATGGGCTGGGAGGCCTCCTCCGAGCGGATGTACCCCGAGGACGGCG CCCTGAAGGGCGAGATCAAGCAGAGGCTGAAGCTGAAGGACGGCGGCCACTACGACGCTGAGGTCAA GACCACCTACAAGGCCAAGAAGCCCGTGCAGCTGCCCGGCGCCTACAACGTCAACATCAAGTTGGAC ATCACCTCCCACAACGAGGACTACACCATCGTGGAACAGTACGAACGCGCCGAGGGCCGCCACTCCA CCGGCGGCATGGACGAGCTGTACAAGTCTAGATCTGCCACCATGCTTGCATTTTGTTATTCGTTGCCCA ATGCGGGTGATGTAATAAAGGGCAGAGTATACGAGAAGGATTATGCTCTATATATTTATCTTTTTGACT ATCCTCACTTTGAAGCTATCTTGGCAGAGAGTGTTAAGATGCATATGGATAGATATGTTGAATATAGG GATAAACTGGTAGGGAAAACTGTAAAAGTTAAAGTGATTAGAGTTGATTATACAAAAGGATATATAG ATGTCAATTACAAAAGGATGTGTAGACATCAATAATAgCGGCCGCCTAaacttgtttattgcagcttataatggttacaaataa agcaatagcatcacaaatttcacaaataaagcatttttttcactgcattctagttgtggtttgtccaaactcatcaatgtatcttatcatgtctGGATCCggectccgcgecg ggttttggcgcctccegcgggcgccccctcctcacggcgagcgctgccacgtcagacgaagggcgcagcgagcgtcctgatccttccgcceggacgctcaggaca gcggcccgctgctcataagactcggccttagaaccccagtatcagcagaaggacattttaggacgggacttgggtgactctagggcactggttttctttccagagagcgg aacaggcgaggaaaagtagtccettctcggcgattctgcggagggatctccgtggggcggtgaacgccgatgattatataaggacgcgccgggtgtggcacagctagt tccgtcgcagccgggatttgggtcgcggttcttgtttgtggatcgctgtgatcgtcacttggtgagtagcgggctgctgggetggccggggctttcgtggccgccgggec gctcggtgggacggaagcgtgtggagagaccgccaagggctgtagtctgggtccgcgagcaaggttgccetgaactgggggttggggggagcgcagcaaaatggc ggctgttcccgagtcttgaatggaagacgcttgtgaggcgggctgtgaggtcgttgaaacaaggtggggggcatggtgggcggcaagaacccaaggtcttgaggcctt cgctaatgcgggaaagctcttattcgggtgagatgggctggggcaccatctggggaccctgacgtgaagtttgtcactgactggagaactcggtttgtcgtctgttgcggg ggcggcagttatggeggtgccgttgggcagtgcaccegtacctttgggagcgcgcgecetcgtcotgtcgtgacgtcacccgttctgttggcttataatgcagggtgggg 
ccacctgccggtaggtgtgcggtaggctttctccgtcgcaggacgcagggttcgggcctagggtaggctctcctgaatcgacaggcgccggacctctggtgagggga gggataagtgaggcgtcagtttctttggtcggttttatgtacctatcttcttaagtagctgaagctccggttttgaactatgcgctcggggttggcgagtgtgttttgtgaagtttt taggcaccttttgaaatgtaatcatttgggtcaatatgtaattttcagtgttagactagtaaattgtccgctaaattctggccgttttggetttttgttagacgctagcggat $A G$ CGCTTCTCATTGGGCATTCCAGCCTACCCAGCTCGGAGTTAGTTACTCCGTAAGTGTGGCCGGAACAG AGTTCGTCCATCTAAAAAGGGAGGGGACCGGCGAACACATCTAGAGCCACCATGGCCACCGAGTACA AGCCCACGGTGCGCCTCGCCACCCGCGACGACGTCCCCCGGGCCGTACGCACCCTCGCCGCCGCGTTC GCCGACTACCCCGCCACGCGCCACACCGTCGAYCCGGACCGCCACATCGAGCGGGTCACCGAGCTGC AAGAACTCTTCCTCACGCGCGTCGGGCTCGACATCGGCAAGGTGTGGGTCGCGGACGACGGCGCCGCG GTGGCGGTCTGGACCACGCCGGAGAGCGTCGAAGCGGGGGCGGTGTTCGCCGAGATCGGCYCGCGCA TGGCCGAGTTGAGCGGTTCCCGGCTGGCCGCGCAGCAACAGATGGAAGGCCTCCTGGCGCCGCACCG GCCCAAGGAGCCCGCGTGGTTCCTGGCCACCGTCGGCGTCTCGCCCGACCACCAGGGCAAGGGTCTGG GCAGCGCCGTCGTGCTCCCCGGAGTGGAGGCGGCCGAGCGCGCYGGGGTGCCCGCCTTCCTGGAGAC CTCCGCGCCCCGCAACCTCCCCTTCTACGAGCGGCTCGGCTTCACCGTCACCGCCGACGTCGAGGTGCC CGAAGGACCGCGCACCTGGTGCATGACCCGCAAGCCCGGTGCCGTCGACAATCAACCTCTGGATTACA AAATTTGTGAAAGATTGACTGGTATTCTTAACTATGTTGCTCCTTTTACGCTATGTGGATACGCTGCTTT AATGCCTTTGTATCATGCGTTAACTAACTAaacttgtttattgcagcttataatggttacaaataaagcaatagcatcacaaatttcacaaataaa gcattttttcactgcattctagttgtggtttgtccaaactcatcaatgtatcttatcatgtctggaattgactcaaatgatgtcaattagtctatcagaagctatctggtctccettcc gggggacaagacatccctgtttaatatttaaacagcagtgttccaaactgggttcttatatcccttgctctggtcaaccaggttgcagggtttcctgtcctcacaggaacgaa gtccctaaagaaacagtggcagccaggtttagccccggaattgactggattccttttttagggcccattggtatggetttttcccetatcccccaggtgtctgcaggctca aagagcagcgagaagcgttcagaggaaagcgatcccgtgccaccttcccegtgccegggetgtccccgcacgetgccggetcggggatgcggggggagcgccgg accggagcggagcccogggcggctcgetgctgcccctagcgggggagggacgtaattacatccetgggggetttgggggggggctgtccetgatatctataacaag aaaatatatataataagttatcacgtaagtagaacatgaaataacaatataattatcgtatgagttaaatcttaaaagtcacgtaaaagataatcatgcgtcattttgactcacg cggtcgttatagttcaaaatcagtgacacttaccgcattgacaagcacgcctcacgggagctccaagcggcgactgagatgtcctaaatgcacagcgacggattcgcget atttagaaagagagagcaatatttcaagaatgcatgcgtcaattttacgcagactatctttctagggttaatctagctgcatcaggatcatatcgtcgggtcttttttccggctca gtcatcgcccaagctggcgetatctgggcatcggggaggaagaagccegtgccttttcccgcgaggttgaagcggcatggaaagagtttgecgaggatgactgetgct gcattgacgttgagcgaaaacgcacgtttaccatgatgattcgggaaggtgtggccatgcacgcctttaacggtgaactgttcgttcaggccacctgggataccagttcgt cgcggcttttccggacacagttccggatggtcagccegaagcgcatcagcaacccgaacaataccggcgacagccggaactgccgtgccggtgtgcagattaatgac agcggtgcggcgctgggatattacgtcagcgaggacgggtatcetggctggatgccgcagaaatggacatggatacccegtgagttacccggcgggcgcgcttggcg taatcatggtcatagctgtttcctgtgtgaaattgttatccgctcacaattccacacaacatacgagccggaagcataaagtgtaaagcctggggtgcctaatgagtgagcta actcacattaattgcgttgcgctcactgccegctttccagtcgggaaacctgtcgtgccagctgcattaatgaatcggccaacgcgcggggagaggcggtttgcgtattgg gcgctcttccgcttcctcgctcactgactcgctgcgctcggtcgttcggctgcggcgagcggtatcagctcactcaaaggcggtaatacggttatccacagaatcagggg ataacgcaggaaagaacatgtgagcaaaaggccagcaaaaggccaggaaccgtaaaaaggccgcgttgctggegttttccataggctccgccccctgacgagcat cacaaaaatcgacgctcaagtcagaggtggegaaacccgacaggactataaagataccaggegtttcccctggaagctccetcgtgcgetctcetgttccgaccetgc cgcttaccggatacctgtccgcctttctccettcgggaagcgtggcgetttctcatagctcacgctgtaggtatctcagttcggtgtaggtcgttcgctccaagctgggctgtg tgcacgaacccccettcagccegaccgctgcgecttatccggtaactatcgtcttgagtccaacceggtaagacacgacttatcgccactggcagcagccactggtaac aggattagcagagcgaggtatgtaggcggtgctacagagttcttgaagtggtggcctaactacggctacactagaaggacagtatttggtatctgcgctctgctgaagcca gttaccttcggaaaaagagttggtagctcttgatccggcaaacaaaccaccgctggtagcggtggttttttgtttgcaagcagcagattacgcgcagaaaaaaaggatctc aagaagatcctttgatcttttctacggggtctgacgctcagtggaacgaaaactcacgttaagggattttggtcatgagattatcaaaaaggatcttcacctagatccttttaaa ttaaaaatgaagttttaaatcaatctaaagtatatatgagtaaacttggtctgacagttaccaatgcttaatcagtgaggcacctatctcagcgatctgtctatttcgttcatccata gttgcctgactccccgtcgtgtagataactacgatacgggagggettaccatctggccccagtgctgcaatgataccgegagacccacgctcaccggctccagatttatca gcaataaaccagccagccggaagggccgagcgcagaagtggtcctgcaactttatccgcctccatccagtctattaattgttgccgggaagctagagtaagtagttcgec agttaatagtttgcgcaacgttgttgccattgctacaggcatcgtggtgtcacgctcgtcgtttggtatggcttcattcagctccggttcccaacgatcaaggcgagttacatg atcccc catgttgtgcaaaaaagcggttagctccttcggtcctccgatcgttgtcagaagtaagttggccgcagtgttatcactcatggttatggcagcactgcataattctctt actgtcatgccatccgtaagatgcttttctgtgactggtgagtactcaaccaagtcattctgagaatagtgtatgcggcgaccgagttgctcttgcceggcgtcaatacggga taataccgcgccacatagcagaactttaaaagtgctcatcattggaaacgttcttcggggcgaaaactctcaaggatcttaccgetgttgagatccagttcgatgtaaccca ctcgtgcacccaactgatcttcagcatcttttactttcaccagcgtttctgggtgagcaaaaacaggaaggcaaaatgccgcaaaaaagggaataagggcgacacggaaa tgttgaatactcat

pBlue 165 mCherry-K3L:

GAGCTCtggcaacctagataaaaatcaactattgttttatccactttctcgtatgttcgaatgagattataatcetgtattatatgggatgtggaagaattggaacacacg cgtgctatataatgaagagataaatatacactccagtcaagtatttcctttttaaaaaaatccatatataatttatattctgtaacatgttatcccttttcaattaacaatgttagtttata aaaaattaaagaagcgaatcaatgattaatagatgttaagaactataattacgatgtattaataggtatatagttagttggttagttaaaaaagagataacagttactaattaattg ttagttattgtctatatgatattacaacctattatttgttctctatagttacattaattaaaattttatatgtgacacccattcatctggagaatacttcttgataccattagtatccatata ccatgattctggccaatctgcaaacacagcacGTCGACCCCTGCAGATCGATTTAAAAATTGAAATTTTATTTTTTTTTTTT GGAATATAAATGGTGAGCAAGGGCGAGGAGGATAACATGGCCATCATCAAGGAGTTCATGCGCTTCA AGGTGCACATGGAGGGCTCCGTGAACGGCCACGAGTTCGAGATCGAGGGCGAGGGCGAGGGCCGCCC CTACGAGGGCACCCAGACCGCCAAGCTGAAGGTGACCAAGGGTGGCCCCCTGCCCTTCGCCTGGGAC 
ATCCTGTCCCCTCAGTTCATGTACGGCTCCAAGGCCTACGTGAAGCACCCCGCCGACATCCCCGACTAC TTGAAGCTGTCCTTCCCCGAGGGCTTCAAGTGGGAGCGCGTGATGAACTTCGAGGACGGCGGCGTGGT GACCGTGACCCAGGACTCCTCCCTGCAGGACGGCGAGTTCATCTACAAGGTGAAGCTGCGCGGCACCA ACTTCCCCTCCGACGGCCCCGTAATGCAGAAGAAGACCATGGGCTGGGAGGCCTCCTCCGAGCGGATG TACCCCGAGGACGGCGCCCTGAAGGGCGAGATCAAGCAGAGGCTGAAGCTGAAGGACGGCGGCCACT ACGACGCTGAGGTCAAGACCACCTACAAGGCCAAGAAGCCCGTGCAGCTGCCCGGCGCCTACAACGT CAACATCAAGTTGGACATCACCTCCCACAACGAGGACTACACCATCGTGGAACAGTACGAACGCGCC GAGGGCCGCCACTCCACCGGCGGCATGGACGAGCTGTACAAGTCTAGATCTGCCACCATGCTTGCATT TTGTTATTCGTTGCCCAATGCGGGcGATGTAATAAAGGGCAGAGTATACGAGAAGGATTATGCTCTAT AcATTTATCTTTTTGACTATCCTCACTcTGAAGCTATCTTGGCAGAGAGTGTTAAGATGCATATGGATAG ATATGTTGAATATAGGGATAAACTGGTAGGGAAAACTGTAAAAGTTAAAGTGATTAGAGTTGATTATA CAAAAGGATATATAGATGTCAATTACAAAAGGATGTGTAGACATCAATAAtaggcgtttaaacaattggaATCAGC TAAAAATTGAAATTTTATTTTTTTTTTTTGGAATATAAatgattgaacaagatggattgcacgcaggttctccggcegcttgggtggag aggctattcggctatgactgggcacaacagacaatcggctgctctgatgccgccgtgttccggctgtcagcgcaggggcgeccggttcttttgtcaagaccgacctgtc cggtgccetgaatgaactgcaggacgaggcagcgeggctatcgtggctggecacgacgggcgttccttgcgcagctgtgctcgacgttgtcactgaagcgggaaggg actggctgctattgggcgaagtgccggggcaggatctcctgtcatctcaccttgctcctgecgagaaagtatccatcatggctgatgcaatgcggeggctgcatacgcttg atccggctacctgeccattcgaccaccaagcgaaacatcgcatcgagcgagcacgtactcggatggaagccggtcttgtcgatcaggatgatctggacgaagagcatc aggggctcgcgccagccgaactgttcgccaggctcaaggcgcgcatgccegacggcgaggatctcgtcgtgacccatggcgatgcetgettgecgaatatcatggtg gaaaatggccgcttttctggattcatcgactgtggccggctgggtgtggcggaccgctatcaggacatagcgttggctaccegtgatattgctgaagagcttggcggcga atgggctgaccgcttcctcgtgctttacggtatcgccgctccegattcgcagcgcatcgcettctatcgecttcttgacgagttcttctgaGCGGGACTCTGGGG TTCGCGAAATGATCGACCAAGCGACGCCCAACCTGCCATCACGAGATTTCGATTCCACCGCCGCCTTC TATGAAAGGTTGGGCTTCGGAATCGTTTTCCGGGACGCCGGCTGGATGATCCTCCAGCGCGGGGATCT CATGCTGGAGTTCTTCGCCCACCCCAACTTGTTTATTGCAGCTTATAATGGTTACAAATAAAGCAATAG CATCACAAATTTCACAAATAAAGCATTTTTTTCACTGCATTCTAGTTGTGGTATCGATaacagcatctttccactttg atagcgcacacgtatgtcgaggtagcctcatccccaggtttatataccttgatgaatcgacacgegtacttgatgtcctctttcttctcacagaaatgcacaacacaaagtcttt tgatatgctttctatatcattctctaccagtctgaggtagtcgtaacccaccgtgaatccaaatactttgtgtgtattattatcaactccaatgaacaaatatccaccetttgtgttg gtaaaagatgagagtataaaggaagctgetgccgtatacgtgttcttagttgettagctgaaacagatgtatgtttaacaattaatagaattaccaaatttgactaatttaccag cctgaagttctgatctattgaagaactcctctaccaatctctcaattgattcagtgtcttccactccatctggatattcaaattcctgcatttctggtctgggactccatccacctga ttccttcagttcgetGCTAGCCATATGGGTACC

\section{Isolate sequences:}

Isolate 1:

caagtcccataccgcaaatggaatatccattagatttcctagatttactaggataagagaggataaaacgtggaaagaatctactcatctaaacgatttagtaaacttgactaa atcttaatagttacatacaaactgaaaattaaaataacaccatttagttggtggtcgccatggatggtgttattgtatactgtctaaacgcgttagtaaaacatggcgaggaaat aaatcatataaaaatgatttcatgattaaaccatgttgtgaaagagtttgtgaaaaagttaagaacgttcacattggcggacaatctaaaacaatacagtgattgcagatttg ccatatatggataatgctgtatcggatgtatgcaattcactgtataaaaagaatgtatcaagaatatccagatttgctaatttgataaagatagatgacgatgacaagactccta ctggtgtatataattattttaaacctaaagatgttattcctgttatcatatctataggaaaggataaagatgtctgtgaactattaatctcatcagacatatcgtgtgcatgcgtgga gttaaattcatataaagtagccattcttcc atggatgtttcctttttaccaaaggaaatgcatcattgattattctcctgtttgatttctctatcgatgcagcacctctcttaagaag tgtaaccgatagctttttcgcaacgggtttgccgccagaacacagctgaagcttcgaggggctcgcatctctcettcacgegccegcegccetacctgaggccgccatcc acgecggttgagtcgegttctgecgectccegcetgtggtgectcctgaactgegtccgccgtctagCTCCTGGGCAACGGTACCGGATCCCTG CAGAAGCTTCTAAAAATTGAAATTTTATTTTTTTTTTTTGGAATATAAATGGTGAGCAAGGGCGAGGAG GATAACATGGCCATCATCAAGGAGTTCATGCGCTTCAAGGTGCACATGGAGGGCTCCGTGAACGGCCA CGAGTTCGAGATCGAGGGCGAGGGCGAGGGCCGCCCCTACGAGGGCACCCAGACCGCCAAGCTGAAG GTGACCAAGGGTGGCCCCCTGCCCTTCGCCTGGGACATCCTGTCCCCTCAGTTCATGTACGGCTCCAAG GCCTACGTGAAGCACCCCGCCGACATCCCCGACTACTTGAAGCTGTCCTTCCCCGAGGGCTTCAAGTG GGAGCGCGTGATGAACTTCGAGGACGGCGGCGTGGTGACCGTGACCCAGGACTCCTCCCTGCAGGAC GGCGAGTTCATCTACAAGGTGAAGCTGCGCGGCACCAACTTCCCCTCCGACGGCCCCGTAATGCAGAA GAAGACCATGGGCTGGGAGGCCTCCTCCGAGCGGATGTACCCCGAGGACGGCGCCCTGAAGGGCGAG ATCAAGCAGAGGCTGAAGCTGAAGGACGGCGGCCACTACGACGCTGAGGTCAAGACCACCTACAAGG CCAAGAAGCCCGTGCAGCTGCCCGGCGCCTACAACGTCAACATCAAGTTGGACATCACCTCCCACAAC GAGGACTACACCATCGTGGAACAGTACGAACGCGCCGAGGGCCGCCACTCCACCGGCGGCATGGACG AGCTGTACAAGTCTAGATCTGCCACCATGCTTGCATTTTGTTATTCGTTGCCCAATGCGGGcGATGTAA TAAAGGGCAGAGTATACGAGAAGGATTATGCTCTATAcATTTATCTTTTTGACTATCCTCACTcTGAAG CTATCTTGGCAGAGAGTGTTAAGATGCATATGGATAGATATGTTGAATATAGGGATAAACTGGTAGGG AAAACTGTAAAAGTTAAAGTGATTAGAGTTGATTATACAAAAGGATATATAGATGTCAATTACAAAAG GATGTGTAGACATCAATAATAgGCGGCCGCCTAaacttgtttattgcagcttataatggttacaaataaagcaatagcatcacaaaAAAA 
AAAAAAAAAAAAAAAAAAAAAAAAAAAAAAAAAAAAAAAAAAAgaagtgtaaccgataataatgttattatatctagacacc agcgtctacatgacgagcttccgagttccaattggttcaagttttacataagtataaagtccgactattgttctatattatatatggttgttgatggatctgtgatgatgcgatagct gataatagaactcacgcaattattagcaaaaatatattagacaatactacgattaacgatgagtgtagatgctgttattttgaaccacagattaggattcttgatagagatgaga tgctcaatggatcatcgtgtgatatgaacagacattgtattatgatgaatttacctgatgtaggcgaatttggatctagtatgttggggaaatatgaacctgacatgattaagatt gctctttcggtggctggtaatttaataagaaatcgagactacattcccgggagacgaggatatagctactacgtttacggtatagcctctagataa

Isolate $2 *$ :

atgattgcgttattgatactatcgttaacgtgttcagtgtctacctatcgtctgcaaggatttaccaatgccggtatagtagcgtataaaaatattcaagatgataatattgtcttct caccgtttggttattcgttttctatgtttatgtcgctattgectgcatcaggtaatactagaatagaattattgaagactatggatttgagaaaaagagatctgggtccagcattta cagaattaatatcaggattagctaagctgaaaacatctaaatatacgtacactgatctaacttatcaaagtttcgtagataatactgtgtgcattaaaccgttgtattatcaacaat atcatagattcggcctatatagattaaactttagacgagatgeggttaataaaattaattctatagtagaacgtagatccggtatgtctaatgtagtagattctaatatgctcgac aataatactctatgggcaatcattaatactatatattttaaaggtacatggcaatatccgtttgatatcactaaaacacgcaatgctagttttactaataagtacggtacgaaaac ggttcccatgatgaacgtagttactaaattgcaaggaaatacaatcacaatcgatgacgaagaatatgatatggtgcgecttccgtataaggatgctaatattagtatgtacct ggcaataggtgataatatgacccatttcacagattctattacggctgcaaaattagactattggtcgtttcaattagggaataaagtgtacaatcttaaactccctaaattttctat cgaaaataagagggatattaagtcgatagccgaaatgatggetcctagtatgtttaatccagataatgcgtcgtttaaacatatgactagggacccattatatatttataaaatg tttcagaatgcaaagatagatgtcgacgaacaaggaactgtagcagaggcatctactatcatggtagctacggcgagatcatctcctgaaaaactggaatttaatacaccat ttgtgttcatcattagacatgatattactggatttatattgtttatgggtaaggtagaatctccttaatattgtttatggatacggtggaaggaatcattattttatttatattgatgggt acgtgaaatctgaattttcttaataaatattatttttattaaatgtgtatatgttgttttgcgatagccatgtatctactaatcagatctattagagatattattaattctggtgcaatatg acaaaattataaaaaatgaaaaaatatacactaattagcgtctcgtttcagacatggatctgtcacgaattaatacttggaagtctaagcagctgaaaagctttctctctagtaa agatacatttaaggcggatgtccatggacatagtgccttgtattatgcaatagctgataataacgtgcgtctagtatgtacgttgttgaacgctggagcattgaaaaatcttcta gagaatgaatttccattacatcaggcagccacattagaagataccaaaatagcttttcgcaacgggtttgccgccagaacacagctgaagettcgaggggctcgcatctct

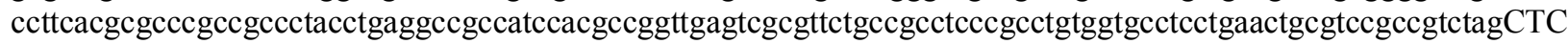
CTGGGCAACGGTACCGGATCCCTGCAGAAGCTTCTAAAAATTGAAATTTTATTTTTTTTTTTTGGAATA TAAATGGTGAGCAAGGGCGAGGAGGATAACATGGCCATCATCAAGGAGTTCATGCGCTTCAAGGTGC ACATGGAGGGCTCCGTGAACGGCCACGAGTTCGAGATCGAGGGCGAGGGCGAGGGCCGCCCCTACGA GGGCACCCAGACCGCCAAGCTGAAGGTGACCAAGGGTGGCCCCCTGCCCTTCGCCTGGGACATCCTGT CCCCTCAGTTCATGTACGGCTCCAAGGCCTACGTGAAGCACCCCGCCGACATCCCCGACTACTTGAAG CTGTCCTTCCCCGAGGGCTTCAAGTGGGAGCGCGTGATGAACTTCGAGGACGGCGGCGTGGTGACCGT GACCCAGGACTCCTCCCTGCAGGACGGCGAGTTCATCTACAAGGTGAAGCTGCGCGGCACCAACTTCC CCTCCGACGGCCCCGTAATGCAGAAGAAGACCATGGGCTGGGAGGCCTCCTCCGAGCGGATGTACCCC GAGGACGGCGCCCTGAAGGGCGAGATCAAGCAGAGGCTGAAGCTGAAGGACGGCGGCCACTACGAC GCTGAGGTCAAGACCACCTACAAGGCCAAGAAGCCCGTGCAGCTGCCCGGCGCCTACAACGTCAACA TCAAGTTGGACATCACCTCCCACAACGAGGACTACACCATCGTGGAACAGTACGAACGCGCCGAGGG CCGCCACTCCACCGGCGGCATGGACGAGCTGTACAAGTCTAGATCTGCCACCATGCTTGCATTTTGTTA TTCGTTGCCCAATGCGGGcGATGTAATAAAGGGCAGAGTATACGAGAAGGATTATGCTCTATAcATTTA TCTTTTTGACTATCCTCACTcTGAAGCTATCTTGGCAGAGAGTGTTAAGATGCATATGGATAGATATGT TGAATATAGGGATAAACTGGTAGGGAAAACTGTAAAAGTTAAAGTGATTAGAGTTGATTATACAAAA GGATATATAGATGTCAATTACAAAAGGATGTGTAGACATCAATAATAgGCGGCCGCCTAaacttgtttattgcag cttataatggttacaaataaagcaatagcatcacaaaAAAAAAAAAAAAAAAAAAAAAAAAAAAAAAAAAAAAAAAAAAA AAagaagataccaaaatagtaaagattttgctattcagtggaatggatgattcacaatttgatgacaaaggaaacaccgcattgtattatgcggttgatagtggtaacatgc aaacggtgaaactgtttgttaagaaaaattggagactgatgttctatgggaaaactggatggaaaacttcattttatcatgccgtcatgcttaatgatgtaagtattgtatcatac $\mathrm{tttctttcagaaataccatctacttttgatctggctattctccttagttgtattcacaccactataaaaatggacacgtggatatgatgattctcttgctcgactatatgacgtcgac}$ aaacaccaataattccettctcttcattccggacattaaattggctatagataataaagacattgagatgttacaggctctgttcaaatacgacattaatatctactctgttaatctg gaaaatgtactattggatgatgccgaaataactaagatgattatagaaaagcatgttgaatacaagtctgactcctatacaaaagatctcgatatcgtcaagaataataaattg gatgaaataattagcaaaaacaaggaactcagactcatgtacgtcaattgtgtaaagaaaaactaa

Isolate 3:

atgaacaaacctaagacagattatgctggttatgcttgctgcgtaatatgcggtctaattgtcggaattattttacagcgacactattaaaagttgtagaacgtaaattagttcat acaccatcaatagataaaacgataaaagatgcatatattagagaagattgtcctactgactggataagctataataataaatgtatccatttatctactgatcgaaaacctgg gaggaaggacgtaatacatgcaaagctctaaatccaaattcggatctaattaagatagagactccaaacgagttaagtttttaagaagccttagacgaggctattgggtag gagaatccgaaatattaaaccagacaaccccatataattttatagctaaaaatgccacgaagaatggaaatatatttgtagcacaacgaatactcccaaactgcattcgtgtt acactatataacaattacactacatttttatcataccactacttcggttagatgttttagaaaaaaataaatatcgcGctttttcgcaacgggtttgccgccagaacacagctgaa

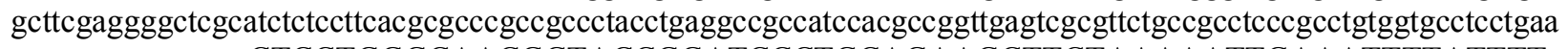
ctgcgtcegcegtctagCTCCTGGGCAACGGTACCGGATCCCTGCAGAAGCTTCTAAAAATTGAAATTTTATTTT TTTTTTTTGGAATATAAATGGTGAGCAAGGGCGAGGAGGATAACATGGCCATCATCAAGGAGTTCATG CGCTTCAAGGTGCACATGGAGGGCTCCGTGAACGGCCACGAGTTCGAGATCGAGGGCGAGGGCGAGG GCCGCCCCTACGAGGGCACCCAGACCGCCAAGCTGAAGGTGACCAAGGGTGGCCCCCTGCCCTTCGCC 
TGGGACATCCTGTCCCCTCAGTTCATGTACGGCTCCAAGGCCTACGTGAAGCACCCCGCCGACATCCC CGACTACTTGAAGCTGTCCTTCCCCGAGGGCTTCAAGTGGGAGCGCGTGATGAACTTCGAGGACGGCG GCGTGGTGACCGTGACCCAGGACTCCTCCCTGCAGGACGGCGAGTTCATCTACAAGGTGAAGCTGCGC GGCACCAACTTCCCCTCCGACGGCCCCGTAATGCAGAAGAAGACCATGGGCTGGGAGGCCTCCTCCGA GCGGATGTACCCCGAGGACGGCGCCCTGAAGGGCGAGATCAAGCAGAGGCTGAAGCTGAAGGACGGC GGCCACTACGACGCTGAGGTCAAGACCACCTACAAGGCCAAGAAGCCCGTGCAGCTGCCCGGCGCCT ACAACGTCAACATCAAGTTGGACATCACCTCCCACAACGAGGACTACACCATCGTGGAACAGTACGA ACGCGCCGAGGGCCGCCACTCCACCGGCGGCATGGACGAGCTGTACAAGTCTAGATCTGCCACCATGC TTGCATTTTGTTATTCGTTGCCCAATGCGGGcGATGTAATAAAGGGCAGAGTATACGAGAAGGATTATG CTCTATAcATTTATCTTTTTGACTATCCTCACTcTGAAGCTATCTTGGCAGAGAGTGTTAAGATGCATAT GGATAGATATGTTGAATATAGGGATAAACTGGTAGGGAAAACTGTAAAAGTTAAAGTGATTAGAGTT GATTATACAAAAGGATATATAGATGTCAATTACAAAAGGATGTGTAGACATCAATAATAgGCGGCCGC CTAaacttgtttattgcagcttataatggttacaaataaagcaatagcatcacaaaAAAAAAAAAAAAAAAAAAAAAAAAAAAAAAAA AAAAAAAAAAAAAagaaaaaaataaatatcgccgtaccgttcttgttttataaaaataacaattaacaattatcaaatttttctttaatattttacgtggttgaccat tcttggtggtaaaataatctcttagtgttggaatggaatgctgtttaatgtttccgcactcatcgtatatttgacgtatgcagtcacatcgtttacgcaatagtcagactgtagttct atcatgcttcctacatcagaaggaggaacagttttaaagtctcttggttttaatctattgccattagtttcatgaaatcctttgttttatccacttcacattttaaataaatgtccacta tacattcttctgttaattttactagatcgtcatgggtcatagaatttataggttccgtagtccatggatccaaactagcaaacttcgegtatacggtatcgcgattagtgtatacac caacagtatgaaaattaagaaaacagtttaatagatcaacagaaatatttaatcctccgtttgatacagatgcgecatatttatggatttcggattcacacgttgtttgtctgagg ggttcgtctagcgttgcttctacgtaaacttcgattcccatatattctttattgtcagaatcgcataccgatttatcatcatacactgtttgaaaactaaatggtatacacatcaaaat aataaataataacgagtacat

Isolate $4 *$ :

gagaaagagaaagagatagttagtctagatattttcttagtacaaaagtcaatgttttaaaatatatggacaagaatttgtctgtataaaaacttgtgtgaaattttgtaccaaag aaaaatgtgagcagtatcccctacatggattttactagatcatttatataccaaaaaatattatacgatctacgttttattatatgattttaacgtgtaaattataaacattattttatg atatacaattgtctggtaacctagatgggcataggggatgttgataagctcgacgagtatatgttgttggacgttattgtttaagaaatagttgatgcatcagaaagagaataa aaaatattttagtgagaccatcgaagagagaaagagataaaactttttacgactccatcagaaagaggtttaatatttttgtgagaccatcgaagagagaaagagaataaaa atatttatgactccattgaagagagaaagagaaaatgagaatgagaataaaaatattttagtgacaccatcagaaagaggtttaatatttttgtgagaccatcgaagagaga aagagaataaaaatattttatgactccattgaagagagaaagagaaaatgagaatgagaataaaaatattttagtgacaccatcagaaagaggtttaatatttttatgagacc atcaaagagagaaagagaataaaaatatttttgtaaaacttttttatgagaccatcaaagagagaaagaGAATAAAAATATTTTTGTAAAACTTT TTTTGTGAGACCATCAAAGAGAGAAAGAGAATAAAAATATTTTTGTAAAACTTTTTTTATGAGACCAT CAAAGAGAGAAAGAGAATAAAAATATTTTTGTAAAACTTTTTTTATGAGACCATCAAAGAGAGAAAG AGAATAAAAATATTTTTGTAAAACTTTTTTTATGAGACCATCAAAGAGAGAAAGAGAATAAAAATATT TTTGTAAAACTTTTTTTATGAGACCATCAAAGAGAGAAAGAGAATAAAAATATTTTTGTAAAACTTTTT TTATGAGACCATCAGAAAGAGGTTTAATATTTTTGTGATACCCTGAAAGGAAATAGGAATAGGAATAG GAATAGTGTCATAATCGTATCACACTATTGAGACAGAAAAAGAAGAAGTAACGAGAGGTAACTTTTTG TGAATGTAGTTAAGAACATTTTTGTTTTGCAAACCGGAATATAGTGTCCGGTACACTTTTAGACCATCG AAGAGAGAAAGAGAATAAAAATATTTTATGACTCCATTGAAGAGAGAAAGAGAAAATGAGAATGAG AATAAAAATATTTTAGTctttttcgcaacgggtttgccgccagaacacagctgaagcttcgaggggctcgcatctctccttcacgcgeccgccgccetac ctgaggccgecatccacgccggttgagtcgegttctgccgectccegcetgtggtgcctcctgaactgcgtccgccgtctagCTCCTGGGCAACGGTAC CGGATCCCTGCAGAAGCTTCTAAAAATTGAAATTTTATTTTTTTTTTTTGGAATATAAATGGTGAGCAA GGGCGAGGAGGATAACATGGCCATCATCAAGGAGTTCATGCGCTTCAAGGTGCACATGGAGGGCTCC GTGAACGGCCACGAGTTCGAGATCGAGGGCGAGGGCGAGGGCCGCCCCTACGAGGGCACCCAGACCG CCAAGCTGAAGGTGACCAAGGGTGGCCCCCTGCCCTTCGCCTGGGACATCCTGTCCCCTCAGTTCATGT ACGGCTCCAAGGCCTACGTGAAGCACCCCGCCGACATCCCCGACTACTTGAAGCTGTCCTTCCCCGAG GGCTTCAAGTGGGAGCGCGTGATGAACTTCGAGGACGGCGGCGTGGTGACCGTGACCCAGGACTCCTC CCTGCAGGACGGCGAGTTCATCTACAAGGTGAAGCTGCGCGGCACCAACTTCCCCTCCGACGGCCCCG TAATGCAGAAGAAGACCATGGGCTGGGAGGCCTCCTCCGAGCGGATGTACCCCGAGGACGGCGCCCT GAAGGGCGAGATCAAGCAGAGGCTGAAGCTGAAGGACGGCGGCCACTACGACGCTGAGGTCAAGAC CACCTACAAGGCCAAGAAGCCCGTGCAGCTGCCCGGCGCCTACAACGTCAACATCAAGTTGGACATCA CCTCCCACAACGAGGACTACACCATCGTGGAACAGTACGAACGCGCCGAGGGCCGCCACTCCACCGG CGGCATGGACGAGCTGTACAAGTCTAGATCTGCCACCATGCTTGCATTTTGTTATTCGTTGCCCAATGC GGGcGATGTAATAAAGGGCAGAGTATACGAGAAGGATTATGCTCTATAcATTTATCTTTTTGACTATCC TCACTcTGAAGCTATCTTGGCAGAGAGTGTTAAGATGCATATGGATAGATATGTTGAATATAGGGATA AACTGGTAGGGAAAACTGTAAAAGTTAAAGTGATTAGAGTTGATTATACAAAAGGATATATAGATGTC AATTACAAAAGGATGTGTAGACATCAATAATAgGCGGCCGCCTAaacttgtttattgcagcttataatggttacaaataaagcaat agcatcacaaatttcacaaataaagcattttttcactgcaAAAAAAAAAAAAAAAAAAAAAAAAAAAAAAAAAAAAAAAAAA AAAAAAtattttagtgacaccatcagaaagaggtttaatatttttgtgagaccatcgaagagagaaagagaataaaaatatttttgtaaaacttttttatgagaccatcaa 
agagagaaagagaataaaaatatttttgtaaaactttttttatgagaccatcaaagagagaaagagaataaaaatatttttgtaaaactttttttatgagaccatcaaagagaga aagagaataaaaatatttttgtaaaactttttttatgagaccatcaaagagagaaagagaataaaaatattttgtaaaacttttttatgagaccatcaaagagagaaagagaat aaaaatattttgtaaaacttttttatgagaccatcaaagagagaaagagaataaaaatattttgtaaaactttttttatgagaccatcagaaagaggtttaatattttgtgatac cctgaaaggaaataggaataggaataggaatagtgtcataatcgtatcacactattgagacagaaaaagaagaagtaacgagaggtaacttttgtgaatgtagttaagaa cattttgttttgcaaaccggaatatagtgtccggtacactttttaattcgtggtgtgcctgaatcgttcgattaaccctactcatccaatttcagatgaatagagttatcgattcag acacacgctttgagttttgttgaatcgatgagtgaagtatcatcggttgcaccttcagatgccgatccgtcgacatacttgaatccatccttgacctcaagttcagatgattcctt gcacatgtctccgatacgaacgctaaactctagattcttgacacattttgtatcgacgatcgttgaaccgatgatatcttcgtaactcactttcttatgagagatgttagacccga gtactggatgggtcttgatgtcgctgtcttctcttcttcgctacatctgatgtcgatagacacctcacagtcttgatcatagccagagcttcttcacgagtgatcgcgggaga gtccttaccttgtcctggggacacgctggacaatctagcattcactgtgtttccatcagcggattctgagatggatttaatctgaggacatttggtgaatccaaagttcattctc agacctccaccgatgatggagtaataagtggtaggaggatctacatcctcgactgatgtggaatcatcttctgattccacctcgggatctggatctgactcggactctgtaat ttccgttacggattggcaaatcttatcattggtcggtgtttggtcttgctttgtgactttgataataacatcgattcccatatgatgtttgtttcttcttccgtacacgaggaggagg atgaggatgattgctgaagactggcaggcacatgcat

Isolate 5:

ttatagtataaagtaataaaaaatagttaatgtgatgactagcgccaccaacgccaacaacatttgataatttctacttactagacgtaccgtaaaaatataaattactataacaa ataatagtatatcaataaacaacctaattaatggtcgaagtatagcaggacattgatgctctagaccgtgtataacaaaatctacaaattttcatccgctatattttgtttcactat atcgtctagacgatcagcgataacttccatgttaatctattaaaatattatcaatatatttcagtttgcatatccgtggtagcaataaccatcggagaagttctaaagaatgtgt ccatgtaagtagtccaatggacgtttccttattggctaaaataagtttgattttatcattggtggatgtgaacagcatacgcttggcatagtacataaacaacgctgccaatatt ataacaccgataacaatcatataaaactgaactcctgtaccagcaacttgtctaggtgctatttgagtagtggecttagtagtcaattgcatcaacgecttaatggcacaatttc ctttgctagatcctgtattaataaattccaaatttgttggagatcctggggctccgtaacattcatctatgattacgttttgtatctttaatttgttatcgacgaccgcgctagaatta caagtttgttttacataattttcaaaatctctaacaacagtgtttacactcgtctgaatgtttaacgcagcagtaaacatagctggcacgtatgctttttgttccggtgttaatccact atatgtttctgtagcggctgataacacagcatccaactgagcatccgcgtccgcagagcacatatttttaacagtgaggttacatccatggttttgtcggatataaaaatttccg atttctatatcacattttgtttgagcactagcattcgcttcttgttctaatttagacgagatacgttcgctgagtgtattcaccgtcgtctgtatgcttgctgcggcacccatttaaat agctacaattagtatccatattaccaagagagataataaactgatcaaatgcaattttaggtcgaacgagtgtttaatattatgttgaactacttttgctctattgacgggaacag tagaaaatctatcactattgcttaatccacatgacaatcttaatgaggaagttttatccatctgtaagttgttcacgctagtattacatcgtacaatattgcaaagtcctaaattatta taattacgtgttagcaagaaattaacattggcattcgaacactctggatcccaacattctcgaggttccgcatattttaatgactcttctaacttatctctagtgggataactacat ctcatatatttctgtttaaagtccgcagactgttgtcttagaatataatcgatcatctcttgctatcttctgtattgtgtgcgcgtaaatgatgcaaaaatgattcacatattggtaca ctagcatctttactacataatgtttgcatcttattatacagatttattaacgattgttgaccctctacagttctattactcctattaaaggctgaaccgatccactgatggcatatgttt ctatcgaacgtatcccctgacaccagtcgaataaatcaacatcgcattttccagtgtcgtgaacgtctggccaacacgattctaatactgcaccctcttcatacttatctgata tctttccatcctttttccaataatgagtacgattaaaagtgcgacagcaattgggtgcggttccattatacgattgtcttaacaatgccgaaagaccaccgggtcctgtgttgac taatctaaactctggatatcttttctttactagctttttcgcaacgggtttgccgccagaacacagctgaagcttcgaggggetcgcatctctccttcacgcgeccgccgecct acctgaggccgccatccacgecggttgagtcgegttctgccgectccegctgtggtgcctcetgaactgcgtcegcegtctagCTCCTGGGCAACGGTA CCGGATCCCTGCAGAAGCTTCTAAAAATTGAAATTTTATTTTTTTTTTTTGGAATATAAATGGTGAGCA AGGGCGAGGAGGATAACATGGCCATCATCAAGGAGTTCATGCGCTTCAAGGTGCACATGGAGGGCTC CGTGAACGGCCACGAGTTCGAGATCGAGGGCGAGGGCGAGGGCCGCCCCTACGAGGGCACCCAGACC GCCAAGCTGAAGGTGACCAAGGGTGGCCCCCTGCCCTTCGCCTGGGACATCCTGTCCCCTCAGTTCAT GTACGGCTCCAAGGCCTACGTGAAGCACCCCGCCGACATCCCCGACTACTTGAAGCTGTCCTTCCCCG AGGGCTTCAAGTGGGAGCGCGTGATGAACTTCGAGGACGGCGGCGTGGTGACCGTGACCCAGGACTC CTCCCTGCAGGACGGCGAGTTCATCTACAAGGTGAAGCTGCGCGGCACCAACTTCCCCTCCGACGGCC CCGTAATGCAGAAGAAGACCATGGGCTGGGAGGCCTCCTCCGAGCGGATGTACCCCGAGGACGGCGC CCTGAAGGGCGAGATCAAGCAGAGGCTGAAGCTGAAGGACGGCGGCCACTACGACGCTGAGGTCAAG ACCACCTACAAGGCCAAGAAGCCCGTGCAGCTGCCCGGCGCCTACAACGTCAACATCAAGTTGGACAT CACCTCCCACAACGAGGACTACACCATCGTGGAACAGTACGAACGCGCCGAGGGCCGCCACTCCACC GGCGGCATGGACGAGCTGTACAAGTCTAGATCTGCCACCATGCTTGCATTTTGTTATTCGTTGCCCAAT GCGGGcGATGTAATAAAGGGCAGAGTATACGAGAAGGATTATGCTCTATAcATTTATCTTTTTGACTAT CCTCACTcTGAAGCTATCTTGGCAGAGAGTGTTAAGATGCATATGGATAGATATGTTGAATATAGGGAT AAACTGGTAGGGAAAACTGTAAAAGTTAAAGTGATTAGAGTTGATTATACAAAAGGATATATAGATG TCAATTACAAAAGGATGTGTAGACATCAATAATAgGCGGCCGCCTAaacttgtttattgcagcttataatggttacaaataaagc aatagcatcacaaatttcacaaataaagcatttttttcactgcaAAAAAAAAAAAAAAAAAAAAAAAAAAAAAAAAAAAAAAAA AAAacttgtctaggtgctatttgagtagtggccttagtagtcaattgcatcaacgccttaatggcacaatttcctttgctagatcctgtattaataaattccaaatttgttggaga tcctggggctccgtaacattcatctatgattacgttttgtatctttaatttgttatcgacgaccgcgctagaattacaagtttgttttacataattttcaaaatctctaacaacagtgttt acactcgtctgaatgtttaacgcagcagtaaacatagctggcacgtatgcttttgttccggtgttaatccactatatgtttctgtagcggctgataacacagcatccaactgag catccgcgtccgcagagcacatatttttaacagtgaggttacatccatggttttgtcggatataaaaatttccgatttctatatcacattttgtttgagcactagcattcgettcttgt tctaatttagacgagatacgttcgctgagtgtattcaccgtcgtctgtatgcttgctgcggcacccatttaaatagctacaattagtatccatattaccaagagagataataaact gatcaaatgcaattttaggtcgaacgagtgtttaatattatgttgaactacttttgctctattgacgggaacagtagaaaatctatcactattgcttaatccacatgacaatcttaat gaggaagttttatccatctgtaagttgttcacgctagtattacatcgtacaatattgcaaagtcctaaattattataattacgtgttagcaagaaattaacattggcattcgaacac tctggatcccaacattctcgaggttccgcatattttaatgactcttctaacttatctctagtgggataactacatctcatatatttctgtttaaagtccgcagactgttgtcttagaat 
ataatcgatcatctctttgctatcttctgtattgtgtgcgcgtaaatgatgcaaaaatgattcacatattggtacactagcatctttactacataatgtttgcatcttattatacagattt attaacgattgttgaccctctacagttctattactcctattaaaggctgaaccgatccactgatggcatatgtttctatcgaacgtatcccctgacaccagtcgaataaatcaac atcgcattttccagtgtcgtgaacgtctggccaacacgattctaatactgcaccetcttcatacttatctgatatctttccatccttttccaataatgagtacgattaaaagtgcga cagcaattgggtgcggttccattatacgattgtcttaacaatgccgaaagaccaccgggtcetgtgttgactaatctaaactctggatatcttttctttactttatcctctttatctttt gctagtggtcctatatgcacatattctaaaagcttagcgggagctatcacgtcatgcattttatccacgtttaataacatctcatcagtgggtactcccggaggcggatcccgt ttagggagctcaacacttactccgccacccatatttatctcattgaaagtattaatctaaaaacgccataaagatgttgatcttaaaggattgaactctatccgaaaacaacatt cctagaatgttatcgtcattatccattacgattctagtttcaaaaacattgactctctttttgaatcctcgtagtttgttgagagacgagatagctattttgaaagaaaacttttgtagt tcttgagaacattcagtcatagaatattccctggaaaatgcatcagtattaccaggagtcttcataataatattgtcatctttaaacataatagccagatgctgatgctgactaata cacttgataaagcccaacaaccattctaaatgaatgacggttctaccacaacatttttcttcatacttgtgaaaattaaacacataagatttcttttgatctatacttagacaaatag tagtatctgtcctaataggcatcagttccttattacaatcgacacttactacatgataactagaaagttttactagattattttccagatcaggttctatatctatgatggcatcattgt gaaaactacacaaacacgattttaccttggacacaggaagattaaacacaatgttttcggctcctcggtagaacactgatgcactgagaggtataatggcccaaatgtttac agatccgcccaaggcggcaaaaatatacattaactcatccgtcgagtctacatttatagacacttcttcactgaactctgaaaaatatgccacaatttggcgcagtttatcgatt tttatacggatgctcattttaaatttttgtaaattatttaaagttaaatggctgcagaacagcgtcgttctacaattttgacatagtttcaaaatgtatagtgcaatctgtattaagag atatatctattaattctgaatacatagagtccaaagctaaacaattgtgctattgtccggcatcgaaaaaggaatcagtgattaatggtatctacaattgttgcgagtcaaatata gaaataatggacaaagagcagctattaaaaatattggacaatcttcgatgtcattcggctcatgtatgtaacgccacagatttctggagactatataattcgttaaaacggttta ctcatactaccgcattctttaatacatgcaagcccactattctagccacg

Isolate 6:

atgccttctttgttctcctcctactaacgaccttagttttccatattttgatttattatcaaattaatttagtaactgtaaatataattatgaattgtttccaagaaaaacaattttcaaga gaaaatttattaaaaatgccgtttagaatggttttaacgggaggatctggatctggaaaactatctatttactatctctgttttctacactagttaaaaaatataaacatatattctt gtttacacccgtttataacccagattatgatggatacatttggccaaatcatattaatttcgttagtagtcaggaatctctagaatataatctgatacgaactaaaagtaacataga aaaatgtattgctgtcgcacaaaatcataaaaatcagcacacttttacttatttttgatgatgtaggcgataaactatcaaaatgcaatactctaatagaattcttaaactttgga aggcatttaaacacgtctattattctactatgccaaacttatagacacgtaccaatattaggacgggctaacattacgcatttttgtagttttaacatttccatctcagacgcggaa aatatgctacgatcgatgcctgtaaaggggaaacgaaaggatatattaaacatgttgaatatgatacagacagctagatccaataatcgattggctattattatcgaagactc cgtatttgtgaaggtgaattacgtatatgtaccgataccgccgataaggacgtcatagaacaaaagttaaacatcgatattttagtaaatcaatattcgcacatgaaaaagaa tctaaacgctatattagaaagtaaaaaacctttttcgcaacgggtttgccgccagaacacagctgaagcttcgaggggctcgcatctctccttcacgcgccegccgecct acctgaggccgccatccacgecggttgagtcgcgttctgccgectccegcetgtggtgcctcctgaactgcgtccgccgtctagCTCCTGGGCAACGGTA CCGGATCCCTGCAGAAGCTTCTAAAAATTGAAATTTTATTTTTTTTTTTTGGAATATAAATGGTGAGCA AGGGCGAGGAGGATAACATGGCCATCATCAAGGAGTTCATGCGCTTCAAGGTGCACATGGAGGGCTC CGTGAACGGCCACGAGTTCGAGATCGAGGGCGAGGGCGAGGGCCGCCCCTACGAGGGCACCCAGACC GCCAAGCTGAAGGTGACCAAGGGTGGCCCCCTGCCCTTCGCCTGGGACATCCTGTCCCCTCAGTTCAT GTACGGCTCCAAGGCCTACGTGAAGCACCCCGCCGACATCCCCGACTACTTGAAGCTGTCCTTCCCCG AGGGCTTCAAGTGGGAGCGCGTGATGAACTTCGAGGACGGCGGCGTGGTGACCGTGACCCAGGACTC CTCCCTGCAGGACGGCGAGTTCATCTACAAGGTGAAGCTGCGCGGCACCAACTTCCCCTCCGACGGCC CCGTAATGCAGAAGAAGACCATGGGCTGGGAGGCCTCCTCCGAGCGGATGTACCCCGAGGACGGCGC CCTGAAGGGCGAGATCAAGCAGAGGCTGAAGCTGAAGGACGGCGGCCACTACGACGCTGAGGTCAAG ACCACCTACAAGGCCAAGAAGCCCGTGCAGCTGCCCGGCGCCTACAACGTCAACATCAAGTTGGACAT CACCTCCCACAACGAGGACTACACCATCGTGGAACAGTACGAACGCGCCGAGGGCCGCCACTCCACC GGCGGCATGGACGAGCTGTACAAGTCTAGATCTGCCACCATGCTTGCATTTTGTTATTCGTTGCCCAAT GCGGGcGATGTAATAAAGGGCAGAGTATACGAGAAGGATTATGCTCTATAcATTTATCTTTTTGACTAT CCTCACTcTGAAGCTATCTTGGCAGAGAGTGTTAAGATGCATATGGATAGATATGTTGAATATAGGGAT AAACTGGTAGGGAAAACTGTAAAAGTTAAAGTGATTAGAGTTGATTATACAAAAGGATATATAGATG TCAATTACAAAAGGATGTGTAGACATCAATAATAgGCGGCCGCCTAaacttgtttattgcagcttataatggttacaaataaagc aatagcatcacaaaAAAAAAAAAAAAAAAAAAAAAAAAAAAAAAAAAAAAAAAAAAAAagaaagtaaaaaacaaa attgtgcaatagcgatcaatcatcgtcgtcaaaaatgtatcatcataattattaacttttgtaacaatagtcctatttagagaaagtctatcgatagacgatcccaatttgtaaatt actccactacttgtcactccatgatatgataaatccatgtaaaatagcatcatctttagatcattaattgttaccttccccaatacaaccaaatcatcatgatatatacctcctccag acaagtatttaacaacggtagaatgctttggcttataaaatacaaatgacattccettatgtttaatcttaatcttttctttagttattgaatcgttacaattataaaatgatgttttttcc aaaacctaagtgtatttaaaatagatgccat

Isolate 7:

atggagggatctaaacgcaaacacgatagtcggcgaccacaacaagaacaggagcagcctcgtccacgtacaccgccatcatatgaagaaattgcaaaatatggacac tcatttaacgtgaaaagatttacgaatgaagaaatgtgtcttaagaatgattatccacgaGcttttcgcaacgggtttgccgccagaacacagctgaagcttcgaggggct

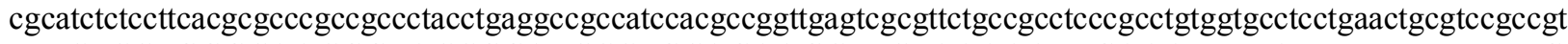
ctagCTCCTGGGCAACGGTACCGGATCCCTGCAGAAGCTTCTAAAAATTGAAATTTTATTTTTTTTTTTTG GAATATAAATGGTGAGCAAGGGCGAGGAGGATAACATGGCCATCATCAAGGAGTTCATGCGCTTCAA GGTGCACATGGAGGGCTCCGTGAACGGCCACGAGTTCGAGATCGAGGGCGAGGGCGAGGGCCGCCCC TACGAGGGCACCCAGACCGCCAAGCTGAAGGTGACCAAGGGTGGCCCCCTGCCCTTCGCCTGGGACAT 
CCTGTCCCCTCAGTTCATGTACGGCTCCAAGGCCTACGTGAAGCACCCCGCCGACATCCCCGACTACTT GAAGCTGTCCTTCCCCGAGGGCTTCAAGTGGGAGCGCGTGATGAACTTCGAGGACGGCGGCGTGGTGA CCGTGACCCAGGACTCCTCCCTGCAGGACGGCGAGTTCATCTACAAGGTGAAGCTGCGCGGCACCAAC TTCCCCTCCGACGGCCCCGTAATGCAGAAGAAGACCATGGGCTGGGAGGCCTCCTCCGAGCGGATGTA CCCCGAGGACGGCGCCCTGAAGGGCGAGATCAAGCAGAGGCTGAAGCTGAAGGACGGCGGCCACTAC GACGCTGAGGTCAAGACCACCTACAAGGCCAAGAAGCCCGTGCAGCTGCCCGGCGCCTACAACGTCA ACATCAAGTTGGACATCACCTCCCACAACGAGGACTACACCATCGTGGAACAGTACGAACGCGCCGA GGGCCGCCACTCCACCGGCGGCATGGACGAGCTGTACAAGTCTAGATCTGCCACCATGCTTGCATTTT GTTATTCGTTGCCCAATGCGGGcGATGTAATAAAGGGCAGAGTATACGAGAAGGATTATGCTCTATAcA TTTATCTTTTTGACTATCCTCACTcTGAAGCTATCTTGGCAGAGAGTGTTAAGATGCATATGGATAGAT ATGTTGAATATAGGGATAAACTGGTAGGGAAAACTGTAAAAGTTAAAGTGATTAGAGTTGATTATACA AAAGGATATATAGATGTCAATTACAAAAGGATGTGTAGACATCAATAATAgGCGGCCGCCTAaacttgtttatt gcagcttataatggttacaaataaagcaatagcatcacaaaAAAAAAAAAAAAAAAAAAAAAAAAAAAAAAAAAAAAAAAA AAAAgaatgattatccacgaattatatcatataatcctccaccaaaatagagaatatatatatcatcatttcatgatgtatactactgacatagtttcaatgtgaacttttcactt tcttgccggttatgaagaatatttttattttaatggtcattactaatcgtatattataattgaaaatgaattagtttaatatgacgctcgtcatgggattctgctgtggtagattctgtg acgctaagaataagaataagaataagaataagaataagaataagaataagaataagaaggaagatgtagaagagggaagagaaggatgttacaattataagaaccttaa tgatctggatgaatccgaagcacgtgtagaatttggaccattatatatgataaatgaagaaaaatcagacataaatacattggatataaaaagaagatatagacacacgata gagtctgtatatttctaa

Isolate 8:

atgatgacaccagaaaacgacgaagagcagacatctgtgttctccgctactgtttacggagacaaaattcaaggaaagaataaacgcaaacgcgtgattggtctatgtatt agaatatctatggttattcactactatctatgattaccatgtccgcgtttctcatagtgcgectaaatcaatgcatgtctgctaacgaggetgetattactgacgecgetgttgcc gttgctgctgcatcatctactcatagaaaggttgcgtctacttttcgcaacgggtttgccgccagaacacagctgaagcttcgaggggctcgcatctctccttcacgcgccc

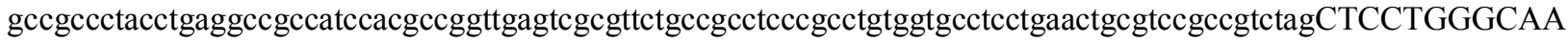
CGGTACCGGATCCCTGCAGAAGCTTCTAAAAATTGAAATTTTATTTTTTTTTTTTGGAATATAAATGGT GAGCAAGGGCGAGGAGGATAAACATGGCCATCATCAAGGAGTTCATGCGCTTCAAGGTGCACATGGA GGGCTCCGTGAACGGCCACGAGTTCGAGATCGAGGGCGAGGGCGAGGGCCGCCCCTACGAGGGCACC CAGACCGCCAAGCTGAAGGTGACCAAGGGTGGCCCCCTGCCCTTCGCCTGGGACATCCTGTCCCCTCA GTTCATGTACGGCTCCAAGGCCTACGTGAAGCACCCCGCCGACATCCCCGACTACTTGAAGCTGTCCTT CCCCGAGGGCTTCAAGTGGGAGCGCGTGATGAACTTCGAGGACGGCGGCGTGGTGACCGTGACCCAG GACTCCTCCCTGCAGGACGGCGAGTTCATCTACAAGGTGAAGCTGCGCGGCACCAACTTCCCCTCCGA CGGCCCCGTAATGCAGAAGAAGACCATGGGCTGGGAGGCCTCCTCCGAGCGGATGTACCCCGAGGAC GGCGCCCTGAAGGGCGAGATCAAGCAGAGGCTGAAGCTGAAGGACGGCGGCCACTACGACGCTGAGG TCAAGACCACCTACAAGGCCAAGAAGCCCGTGCAGCTGCCCGGCGCCTACAACGTCAACATCAAGTTG GACATCACCTCCCACAACGAGGACTACACCATCGTGGAACAGTACGAACGCGCCGAGGGCCGCCACT CCACCGGCGGCATGGACGAGCTGTACAAGTCTAGATCTGCCACCATGCTTGCATTTTGTTATTCGTTGC CCAATGCGGGcGATGTAATAAAGGGCAGAGTATACGAGAAGGATTATGCTCTATAcATTTATCTTTTTG ACTATCCTCACTcTGAAGCTATCTTGGCAGAGAGTGTTAAGATGCATATGGATAGATATGTTGAATATA GGGATAAACTGGTAGGGAAAACTGTAAAAGTTAAAGTGATTAGAGTTGATTATACAAAAGGATATAT AGATGTCAATTACAAAAGGATGTGTAGACATCAATAATAgGCGGCCGCCTAaacttgtttattgcagcttataatggttaca aataaagcaatagcatcacaaaAAAAAAAAAAAAAAAAAAAAAAAAAAAAAAAAAAAAAAAAAAagaaaggttgcgtct agcactacgcaatatgatcacaaagaaagctgtaatggtttatattaccagggttcttgttatatattacattcagactaccagttattctcggatgctaaagcaaattgcactgc ggaatcatcaacactacccaataaatccgatgtcttgattacctggctcattgattatgttgaggatacatggggatctgatggtaatccaattacaaaaactacatccgattat caagattctgatgtatcacaagaagttagaaagtattttgtgttaaaacaatgaactaa

Isolate 9:

gtaaaattaaattaattataaaattatgtatatgatttactaactttagttagataagttagtaatacataaattttagtatattaatattatattttaaatattttatttagtgtctagaaaa aaatgtgtgaccaacgaccgtaggaaactctagagggtaagaaaatcaatcgcGctttttcgcaacgggtttgccgccagaacacagctgaagcttcgaggggctcgc atctctccttcacgcgecegcegectacctgaggecgecatccacgecggttgagtcgegttctgecgectecegectgtggtgectcetgaactgegtccgecgtcta gCTCCTGGGCAACGGTACCGGATCCCTGCAGAAGCTTCTAAAAATTGAAATTTTATTTTTTTTTTTTGGA ATATAAATGGTGAGCAAGGGCGAGGAGGATAACATGGCCATCATCAAGGAGTTCATGCGCTTCAAGG TGCACATGGAGGGCTCCGTGAACGGCCACGAGTTCGAGATCGAGGGCGAGGGCGAGGGCCGCCCCTA CGAGGGCACCCAGACCGCCAAGCTGAAGGTGACCAAGGGTGGCCCCCTGCCCTTCGCCTGGGACATCC TGTCCCCTCAGTTCATGTACGGCTCCAAGGCCTACGTGAAGCACCCCGCCGACATCCCCGACTACTTGA AGCTGTCCTTCCCCGAGGGCTTCAAGTGGGAGCGCGTGATGAACTTCGAGGACGGCGGCGTGGTGACC GTGACCCAGGACTCCTCCCTGCAGGACGGCGAGTTCATCTACAAGGTGAAGCTGCGCGGCACCAACTT CCCCTCCGACGGCCCCGTAATGCAGAAGAAGACCATGGGCTGGGAGGCCTCCTCCGAGCGGATGTACC 
CCGAGGACGGCGCCCTGAAGGGCGAGATCAAGCAGAGGCTGAAGCTGAAGGACGGCGGCCACTACG ACGCTGAGGTCAAGACCACCTACAAGGCCAAGAAGCCCGTGCAGCTGCCCGGCGCCTACAACGTCAA CATCAAGTTGGACATCACCTCCCACAACGAGGACTACACCATCGTGGAACAGTACGAACGCGCCGAG GGCCGCCACTCCACCGGCGGCATGGACGAGCTGTACAAGTCTAGATCTGCCACCATGCTTGCATTTTG TTATTCGTTGCCCAATGCGGGTGATGTAATAAAGGGCAGAGTATACGAGAAGGATTATGCTCTATATA TTTATCTTTTTGACTATCCTCACTTTGAAGCTATCTTGGCAGAGAGTGTTAAGATGCATATGGATAGAT ATGTTGAATATAGGGATAAACTGGTAGGGAAAACTGTAAAAGTTAAAGTGATTAGAGTTGATTATACA AAAGGATATATcGATGTCAATTACAAAAGGATGTGTAGACATCAATAATAgGCGGCCGCCTAaacttgtttatt gcagcttataatggttacaaataagecaatagcatcacaaaAAAAAAAAAAAAAAAAAAAAAAAAAAAAAAAA .............aaaa atcaatcgctttatagagaccatcagaaagaggtttaatatttttgtgagaccatcgaaggagaaagagataaaacttttttacgactccatcagaaagaggtttaatatttttgt gagaccatcgaaggagaaagagataaaacttttttacgactccatcagaaagaggtttaatatttttgtgagaccatcgaaggagaaagagataaaacttttttacgactcca tcagaaagaggtttaatattttgtgagaaaggagaaagagataaaacttttttacgactccatcagaaagaggtttaatattttgtgagaccatcgaaggagaaagagataa aactttttacgactccatcagaaagaggtttaatatttttgtgagaccatcgaaggagaaagagataaaacttttttacgactccatcagaaagaggtttaatatttttgtgaga ccatcgaaggagaaagagataaaacttttttacgactccatcagaaagaggtttaatattttgt

Isolate 10:

atggatatctttaaagaactaatcgtaaaacaccetgatgaaaatgttttgatttctccagtttctattttatctactttatctattctaaatcatggagcagctggttctacagctgaa caactatcaaaatatatagagaatatgaatgagaatacacccgatgacaataatgacatggacgtagatattccgtattgtgcgacactagctaccgcaaataaaatatacg gtagcgatagtatcgagttccacgcctccttcctacaaaaataaaagacgattttcaaactgtaaactttaataatgctaaccaaacaaaggaactaatcaacgaatgggtt aagacaatgacaaatggtaaaattaattccttattgactagtccgctatccattaatactcgtatgacagttgttagcgecgtccattttaaagcaatgtggaaatatccattttct aaacatcttacatatacagacaagttttatatttctaagaatatagttaccagtgttgatatgatggtgggtaccgagaataacttgcaatatgtacatattaatgaattattcgga ggattctctattatcgatattccatacgagggaaactctagtatggtgattatactaccggacgacatagaaggtatatataacatagaaaaaatataacagatgaaaaattt aaaaa tggtgtggtatgttatctactaaaagtatagacttgtatatgccaaagtttaaagtggaaatgacagaaccgtataatctggtaccgattttagaaaatttaggactta ctaatatattcggatattatgcagattttagcaagatgtgtaatgaaactatcactgtagaaaaatttctacatacgacgtttatagatgttaatgaggagtatacagaagcatcg gccgttacaggagtatttacgattaacttttcgatggtatatcgtacgaaggtctacataaaccatccattcatgtacatgattaaagacaccacaggacgtatactttttatagg gaaatactgctatccgcaataaatataaacaaatagacttttatcacgtttatctatgtctaaatattacaaatagtaatagtataaactaaagctgataatacttaaaaaaataata atatcatttacaattaatagtataaactaaaaattaaacaaatcgttattataagtaatatcaaaatgatgatatacggattaatagcgtgtcttatattcgtgacttcatccatcgct agtccacccattacggaagataaatcgttcaatagtgtagaggtattagtttccttgtttagagatgaccaaaaagactatacggtaacttctcagttcaataactacactatcg ataccaaagactggactatccacacctgatggtttggatataccattgactaatataacttattggtcacggttactataggtcgtgcattgttcaaatcagagtctgaggatat tttccaaaagaaaatgagtattctaggtgtttctatagaatgtaagaagtcgtcgacattacttactttttgaccgtgcgtaaaatgactcgagtatttaataaatttccagatatg gcttattatcgaggagactgtttaaaagccgtttatgtaacaatgacttataaaaatactaaaactggagagactgattacacgtacctctctaatgggggttgcctgcatacta tcgtaatggggtcgatggttgattattgattagtatattcctgcttttcgcaacgggtttgccgccagaacacagctgaagcttcgaggggctcgcatctctccttcacgcgc

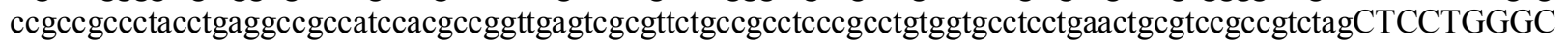
AACGGTACCGGATCCCTGCAGAAGCTTCTAAAAATTGAAATTTTATTTTTTTTTTTTGGAATATAAATG GTGAGCAAGGGCGAGGAGGATAACATGGCCATCATCAAGGAGTTCATGCGCTTCAAGGTGCACATGG AGGGCTCCGTGAACGGCCACGAGTTCGAGATCGAGGGCGAGGGCGAGGGCCGCCCCTACGAGGGCAC CCAGACCGCCAAGCTGAAGGTGACCAAGGGTGGCCCCCTGCCCTTCGCCTGGGACATCCTGTCCCCTC AGTTCATGTACGGCTCCAAGGCCTACGTGAAGCACCCCGCCGACATCCCCGACTACTTGAAGCTGTCC TTCCCCGAGGGCTTCAAGTGGGAGCGCGTGATGAACTTCGAGGACGGCGGCGTGGTGACCGTGACCCA GGACTCCTCCCTGCAGGACGGCGAGTTCATCTACAAGGTGAAGCTGCGCGGCACCAACTTCCCCTCCG ACGGCCCCGTAATGCAGAAGAAGACCATGGGCTGGGAGGCCTCCTCCGAGCGGATGTACCCCGAGGA CGGCGCCCTGAAGGGCGAGATCAAGCAGAGGCTGAAGCTGAAGGACGGCGGCCACTACGACGCTGAG GTCAAGACCACCTACAAGGCCAAGAAGCCCGTGCAGCTGCCCGGCGCCTACAACGTCAACATCAAGTT GGACATCACCTCCCACAACGAGGACTACACCATCGTGGAACAGTACGAACGCGCCGAGGGCCGCCAC TCCACCGGCGGCATGGACGAGCTGTACAAGTCTAGATCTGCCACCATGCTTGCATTTTGTTATTCGTTG CCCAATGCGGGcGATGTAATAAAGGGCAGAGTATACGAGAAGGATTATGCTCTATAcATTTATCTTTTT GACTATCCTCACTcTGAAGCTATCTTGGCAGAGAGTGTTAAGATGCATATGGATAGATATGTTGAATAT AGGGATAAACTGGTAGGGAAAACTGTAAAAGTTAAAGTGATTAGAGTTGATTATACAAAAGGATATA TAGATGTCAATTACAAAAGGATGTGTAGACATCAATAATAgGCGGCCGCCTAaacttgtttattgcagcttataatggtta caaataagcaatagcatcacaaaATCACAAATAAAGCATTTTTTCACTGCAAAAAAAAAAAAAAAAAAAAAAAA AAAaagagtaacagctgccccattcttaataatcgtcagtatttaaactgttaaatgttggtatatcaacatctaccttatttcccgcagtataaggtttgttgcaggtatact gttcaggaatggttacatttatacttcttctatagtcctgtctttcgatgttcatcacatatgcaaagaacagaataaacaaaataatgtaagaaataatattaaatatctgtgaatt cgtaaatacattgattgccataataattacagcagctacaatacacacaatagacattcccacagtgttgccattacctccacgatacatttgagttactaagcaataggtaata actaagctagtaagaggcaatagaaaagatgagataaatatcatcaatatagagattagaggagggctatatagagccaagacgaacaaaatcaaaccgagtaacgttct aacatcattattttgaagattcccaaataatcattcattcctccataatcgttttgcatcatacctccatctttaggcataaacgattgctgctgttcctctgtaaataaatctttatca agcactccagcacccgcagagaagtcgtcaagcatattgtaatatcttaaataactcat 
*Note that some isolate 4 viruses carry a second insertion that precisely matches that of the isolate 2 insert. We believe that this is a result of recombination between two separate strains during plaque purification of both.

Nanopore reads showing K3L-CNV:

Isolate 4:

TGTTGATAATGGGAAGATGGGATATATGATGTTGCATGAACGTTGATAGTTCACTGGTCATCAATATG CCGACATTCAATGCCCAGAATCATGGTTAATTTCGCCAATACATACCACGATACTTCCAGCGTGAGAA GTCGCGTAAACAATATCCTCATCTCCTCTGCGGACGCTATTAATTGTGATAGTTGAATACCAGCCAGCG CTTGTAATTTCGGCGTCGTTAGCGCAGTCACTGGTTTGAATGATAGCAGCAACGTCAACATATTTCATC ATTTTATGAGGATTCGTGCTATGCCAGCCAGCGTGTGTTTATAACAATATTTCGTAGCCCATATTATTA ATTCAGTAATATAAACTATCAATATTGTGTTACTGTAAAATGTCATGCGGTCAAATGTTATTAGTCGTA CACGCATGCCAATATAATATAGTCGTAATTATGCGCAACACGGAAGATATCACCATTTGTCATTGTCG CTTCATCATATCTTGAGCTTAGAACATCATCAATCCATTTCATGCCGGTGACACGGTATGCACAAGCGA TTGATTGCACGCAATACAACAATGAAGTATGAATACGGGGTACAAACAACCGAAATTTCAACATTACT ATTTATGATATACACGTTATGTCGCCATTGCTTCATACCATGGATTGTGAATCAATCGTAAACAATTTT CTGCTTATTTTTTATTACTTTTTATTCGTTTGCCCATATAGGAACAACTATCGTACAGCATAGTCGGCGA ATGGCATCGATAACGACGAGTCATTTATGCAGGACGCGCAAGATATTCCGTACAGCATCATACGCAAC AAAGCGTACGGGCGAAATAAAAGACATTTTCAACCGGAACTTTACACGCTACCAAACAAAGTGACCA ATCAATCTAGCGGTAAAAGTCCGCCATTCACGTATGACAGCCAAAGGCGCCGCCCATTTTGGGTACGC TTTCAAACAGCCCACATAGTACAGACAAGTTTTTATATTTCATTAACATGGTTGCGAAGGCCTCGTGGG TCATGCAGTTGAGTATTACGACTGAGTCAGTGACACTTTCATTATCGATACCATGAACTTCAGTATACG ATTATTTATGTAGCTGCGATCAAGATGAAAAATTTGCGTCGGTGCGGTATGTTAGACTTCGTTAAAAGC TTGGAAAGTGAAATGACAGAATCGCATGGGCGTCGCATTTTGAAATTTAGACTTTTTCAATACATTCG GACATTGGCGCATTTGCTAAAGATGCGTAATGTCACTGCAGTAGGCCTTACATACGACGTTACAGATG TTACGAGGAGTGACATCGGCCGCCACAGGAGAGTTTACGATTAATCTCTTTCGATGGCACTACGTACA TGATTAAAGACACCACAGGACGCAAGGTTTTATAGAAATACGTTATCCGCACAAATATGTTCGAATAA AATATTTCATCACGTTTGTCTAAATATTACAAATAAAGTGGGTCAGTAGCAACTAAAAAGCCGACAAT GGTTTCAAAAATAATATCATTTTACTACCACAGGAAACTGTACTAAACAAACGTTATTAGTAATATCA AAATGATGACATACGATTATCAGCGCAGACTTTGAAGATGTAACAAGTCAATAGCGTAGAGGCATAGT TTCGAAAGTTTGAGATTTATCAAAAGAATATACGGTAATCAGTTCACAATCACAATTGTCGATAAATA AAGACTGCCATTCATCACCCGACGGGATGAGTGTCATCGACTGGTCAGATAGCTGGTCACGCATTATA GGCCATCATTCGAGTTTCCAAAGAAATGACCAGGCATAGCGTAAGAAGTCGCACGACAGGACTCGAG TATAAATTATCGCGCATAGCCAAGTTGTTACTTATCGTATGCTCCATTTGCCGACATTAGCATATGCCC CTACTCACACAAAAAGAACATTTTTTATAAACATGATCTGAGAGGCTTAGAGCAATCCAGCATGTTTA ATATGCAACATTTCGCTGTTTCATAAAATGAAGGTATAGTCATTAATATGACGCTTAGTTTACATCGGC AATCATACCATTGCCCCGGCCATACTATTTGGACAGCATTAGCGTTCGTGGCAAAGAAAACATGCATG GCGACATTGGACTGGTTATTATCAATAAAGAAGAGTGTAGTGTCAAAAGTAAAGTCAACAAAAGTAA CGGTATCGTTGGCCGCTGAGCTTGACTGACAGACATACGAGGGTGTTAGAATGTCCATTAAAATATGT GACATCAACGGAACATAATATATAAATGCACATTGCCGTTGGCATAATTGCCGATTTCCACGAGTTAA AGTTATTCAATATGAACTTCACGCGACCATACTGTCGATGCATACACTGTTATTACCACGAAGAATCAT GCGACTCTCTACATCTCATCGATGATAACATGTACAATATTTCTCGAATAAGTCTTTTAAATAATATGA AAAACTACATGCAGATGCAAAGATGTTTAATGATGATACGATTTCATCTTCAGCGAGAAGATGTCGTG CCAGAATACTTGTCATAACCGTTTAATAATATCAATTTAGTCACGATAATACAACATTTGTTAAGTTCA AACTTTCCACAGCCAGACTGCTTGTAGAATGCCGTGACATAAACAGTCCAGTAGAAGGTACATTCCCA CACACTGTTAATTCGCTGGTCGAATTATGATTTGACCATTTTAAAACCTTTCAAGGAGTATCAGCCCAC TCATTATCATGATGAAGTTGCGAATTATATGCTTTCTCTCGATAGTATAGATAGCTTAGCTAATGTATA ACATAATTCACCATAGCAGTTTCACCGATTTAAAACGATAAACTATTGATTACGCACTGTATAAGTTGC CGGGAAAAGGAGCAGACCCCAATATGTAGATTATAGAGGGCTTCTTCTGCCACGCCTCGCATCTATAT GTTCATTCTCATGGAAAAGATGGTCGCAGTGCCATTTGTTGTAAGAGTTAAAACAGAGTCCTGAAAGT ACCTCATAACTACGCAGCTCCCTCAATATTGCTCTCACCTCCTTCATCTCACAACCCACAAAAATGGAA ATAAAAGCCCTCATTGGGCATAAACGCCTGGGTTGCCTTCTCGCCTGCTCAGACCCTCGCCAGCCGGC GACCCCTACGTGTCGCATGTCTGACTGGTCACACCTCGTGTTTCATAGAGACCCTGCAGCTCCTTCAGC CTCATTCACAACGAGCTTGGCGGTGAAGCCGAGTACTGCGGACAATTGCGTTATTAAATGTTTCATCA GAATGAAACCGCCGAAATATTGCATCATTGACTTTGGTCATCAATATCGTTCCCGGCCAACGATGGGT ATAGTCGACATACATGCCGCAGGGTAATCCATAGTCATGCCACTGGGTTGACTTTTTGTCATTCATGAG 
ACGATACGCTTTCGTGAAATAGAAGGACAGCGTTGGCGATTATTGCGGAAAGCACCAGCTTCATGCGT CGATGAAGATGCAAACTGAGCATTGAAACGTCACGGGGTCTCATCGAGCAGACAGCATCTATAAGTC GGTCGCACATGTCTCCACACGCGATACAGACAAATTCGACTGCCTGCGAGTCGAGAGAGTCAGGAAA CAACGAAGCGATAACTCGCCAGCGATGCCAGTGTTACAGACATGCAAAATTCAGACAGTACTCCACG ATGTCCGGTACATTTCATTTCAACGAATGATTATGATGTCCGTTCAGAAAGATGCGAGGCGCTTAGAA AATTCCCCATATACGAGGAAACGTTAATCTTCTTACGTCATGGTTTCGTCGATGCAATGCGGTACAGCC ACATCTGGCAAGGTCGCGTCTCCACTGGCTTGGTCATTTCAAGAGATGAATAACAAATCCAGTTACGT TGATAAACTACTGATGCGACAGGGTCGTTCAGATGGACGACAACAATAATTTTGTTAATTAAACGTAA GGTTATCGCGGAAATCAGATACGCGGCTAAAATCACACAATTATGCTATTGTCGAGATTTACTGCAAG GTCACTGTTATGAAACCATACTACGGCTTGATATACCACAAGTACATGGACGATGACATGACGACAAT AAAATCATTATAGTTACGCGGTCGATGTCAATAATATACAATTTCAAAGCGGTTGGTTATGGAGCATC AATGCCGATAATAACAGCGTCATTCAGTAGCAGTTGCCAAAGAGAAAAATAAAATCTATGGAGAAGC CGATTTTATTTCACAGTCATCCAGAGACAGACGAGCCTGGGTCGATGCGCATGCTGCCTATATCTCGA AATCATTCGGGTATTGTCACGCCCAGATGATTTTTGCAAAAGATATCGCCGGTCATAACTGTGCTATAG AGCAGACATTAATAGAATGAAGAATGCCTGGCTATTGGTCAGGCGTTCCATGCTTGATATATGCTTAA ACGAAAGAAACGCCACAGACTGAGGATACGCAAGCATTACATCAAATGGTACAGTAATCCACCCAAA GTCCATCACAGAAAACCGTACACGAATATTACAGACGATATTCCTGATTTTCGAAATTTCACCATTCAT TGCCATCACGCGGAGCGAGCTGGTCGTCTCAGCCAGCTTCATGATACAGTTGAACGATGATAAAAGAT ATCGGCATCATAAACACGGGAGTTTGTTTATATGCAAAATCCGCATAAACATTAAAAAATATATTGTA CCTGTTTCTTTCTTTTCGTACATCTTCTACTGCGAAAAGTAAATGATGCAAAGGAGATGGGCGATGAGA TTGAATAAACCGCATTGCGTTATACCTAACGTAGCAGGACGATGTCACGCTTTCAATGTTTGTCAACAT AAGGGTTTACGTTTCGATGTGGACAAAGACCATGTTTAAGCGTTGTTTATTCGAATAAACAAAATCCTC CATTTACCGCCATTGTTTAAATGCCACGGTTTGGTCCATTCGTACAGCATTGGCACAAATACAACAGCA ACCCGATAGCGATGCAGGTGCCACTACTCGAGCCCATTGTCATCAATGAGCTGTCAAAGGTTTCTTTCG ATACACGACGTTCTTTAAAACATCGATCTGTTGCTGCAAGTTATTTTACCGTCGGGAAAGCAAATTGAT TCATTTAATTGGCTCTTTCATTGGTCATTGCAAAACACATGTTCACGTTCTCGTGCTCATTGTTGTGTTA CATAGAAATTGCCGCGTGAGCAAACAGATAACGTAAGTCAGCACTACCTTCAACAGCATGTCAAACAT GTTCTTCGCATGCTCTTTTGCCCGAGTGACAGCGGCCACCGTGCCAATTGTCATGTAAGTTACAGCTCT CTGCTCGGCATCAAAGGAAGAAGGACACCACTTTGCCCATCATAAAACATATCGCGATACACTCAATA TACCACGGCATTCGCCAGAGAAGACGGCCACAATACGCTCAAAATTAAAATATACATATGAATATCAA CCTTCCGATGATCGCTCGACTTTCTGAACAGAAACATCGTTATTAAGCAAGTGATGTTGTGACGACAC ACACAGACTGTATTACCCATGATTAAATGTTACAAGATGCTCAGAGTTCTCGTCGATTACTGCAGCCG AAGTCATCGTTTACAGTGATTTATCATTTTATAAAATTGCTTAAATGAGATTATAAAAGTGCATTATTG AAAATATCATTCTCGACAAGATAGATTCTGCCTGTCGCGACATAAGTCGCTCAAAGTTATATTACCCCG TATGCTTGCTTCAAAGTTGTCTTGAAATATGGGGACAGAGTCAATATTACTTCCTTCTCATACGACGAT CCGGTCAGTGTCATCTGTCGATGGAAAGCGTAAACCCAAAATGACAAAGTTACCAATAAATTGGGAA AGTTCACATCTGGCCGTATCATGCGCACACACCAAAGAAAGGCAGCTGTATTTGAAAGAAATCATTGC GTCATTATTCATGACAGAAGAAAATGAATTGTTGGAGAAGTAAAAGACAATATTACAATTATGAATAT ACTGTATATTGCTCTCTCATGCATATCAAAGACAAACATCCGATGGAAAGTGGAAGACAATTTAACAA ACGTTACGGGTTTCAGTCTGGGGAACATGCTTCCAGACAGCAGGTTAAATGGTCGCCAAAGCCGTGTA ACACTGCAATAATACCGTTGTCGCCGTATGGAAGACGAGTCAAACATCTTCACAATAAGTCAGGCCTC CGTGAGCTCTCCCCGGATATTGCCCGCATGCTTTCAACAAAATGTGAAGGATTACGGAGAACAGCCCG GCTCGTGTTCGGAATATCAAACTTCTTCGGCAGAATCACAGAAATGTTTCCACCATACGATGCGGAAA TTAACCAACGCCGACGACACTGGCGCTCGCGACCACAATGCCGGAAATTATTGCTTTGCCAAAAGATC TCAAGAGCCAAGATGGATATTCTCAGGATTTTCACAGGACCCCGGAATTTCGAGTCAAAGGCAACAAA GATTCTTCTCCAAATAATCTAGTAAAGACAGAACATGTCAGCAGGAGACTGCCTATCATTCATACTAT GCGGGTCAATAATACTACATTTGAAAACCGATACAATCTCTTACTTTTCTATGTCGAGGTTATTATGCT CGGTATTAGCGATGACCCCGCTATAGTAAGTTTTTCACCATAACAATACAATAATAATTTCGTAAAAG CAGAAAATATATTCTGGTTCTGTACGGCAGCGAAGCAGAATATGTTAACAATAAAGGGCCGGCGAAA GAATATACGTCTCGGTGATGGATGTTCGCAATAGCCTCGCAAATCATCTCATTCTTCCTTTTCGCATGG AAGAAGAAAACAAACATCATGAAGGGGAACGATGTTACATCAAAAGTCACAGTCACTTTATCATGAT AAGATTTGCCGACCGCATAGAGTTCTGAGTCAGTTCAGATCTCTTTATAGTGGGAACAGAAGATGATC AACATCAAATGAGATTCTCATCATCTGATACCATCATCGGTGAGGTTCAGCGAACGGATCACCAATGT CCAGACACCATTCAGAATCCGCCGATTGAAACACAGTGAATGGCACAGTCTGGGTACCTCGCTTTCTC TCGCGATCACCAGAAAGAAGTTTCGCTGTCAAAGACTGTGAGGTGCTCCACGACACGACAAGACAGC GACACCATTCAGGTTCAACATCATACAGTGAAAGATCGGTTCAACATCGACAAAATGCATCAGAATCT 
CAGAAGTTTGAAGTAGACAGTAATCACCTGACCTGAGTCGGCGATGGATTCTACGTCGACGATCGGGC ATTCGAAGGCAACCGACTCGTCGATCAACAAAGTGTGTTCGACGATAATTTGTTCATTCGAAATTGGA TAATCGAACGATCAGGCACACCACGAATTGCAAAATTATATTTGCTTCAAACAAAATGTTCTAATTGC CATCTTCACAAAAGTTATTTCGTTACTTCTTTTCTGTTCAATAGAAGATTGTCAATTATTCCTGTTCCCC TATTCTTTGCTTCCTCGGCCAAAAATATTAAACCTTCCTTTTCGATGGTTCATAAAAAGCTTCAAAATA GTTTTATTCTTCTTTGATAACTGATAGGGTTTTATTGTGTATGATTTTGATCCATAGGGAAGTTTGTAAC ATTTTTAAGTCCAGGGAAGTTCTTGACAGTTTTATGTCTCTGATGAACCATAGGGTCTTTATTTTATCTT CATAGGCCTATGTGACAAAATATTTATTCTGAGTCCTCATTGTAACGTTTATTTGAATTTCTGATTGCCG TGAGGTCGTCTATTGATAAACCATTGTCATCCCAGGGACTGACCATAATTTATCCTGACCCTTATGTTT TCCCGTCATTTGTTCATATCAACATGTCTATTCATGGCCTGACAAACAAGATTAAAGTGAGACAGTCAT AGCATAATACTTCGCCTTTATCACATCACCGCACAAGGGACGAATGTCGAATGCAGCATGGCGGCAGA TCTGACTGCAGTTTCGTTCATGCCAGCGTACTGTACGTTCATCATGGTAGTCTCGCCGCGGGGGTGGGT GACGTCGACGCCGGGCAATGGCTTCGGGTTGTGCAGACCAGCGTCGCAGTGGCCGCCGCCCTGTTTTC AAAGTTGACTGCCCTTTCAGGGCTGACCGAGCCTCCAGTTCATTGTTTTCCCGCGAGTTTGTAAGTCGT CGGAGGAGAAAACAGCAAAGCCTGTAGTAATCCGTCCTGCAGGAGTCTCGGGTCGCGCCCACGCGCC CTCATTCTTCCTCGGTAGTCGGGGATGTCGGCAGCAAGCCACGTAGTTCGAGCCGCATTACGAACTAT GAAAGGTGTTGGCCAGTTTTGCGCGAATTTGCCAGGTCTCGCCCGCTCGCCTGGGCGCATTTTTGATGA TGGCCATGTTTCCTCGCCCCGTCACCACTTCATATTCAAAAAAAAAATAAAATTAATTTTTAGAGTTTT TCTGTAGGGGATTCGGAATAAGCAGTTCAGGAGATTTATGATGAGCCAGAGGTACCACAGGGGAGGT GGCAGAAGTTAGTAAGGGCGGGCAGAGATGTGAGCTCGAGTTGCCGTGTTCTGGAAAGTGCAGTAAC CCGTTGCGAAAGACCAAATATTCTCATTATTTTCTTTCAATGGAACATAAAAATATTATCTCTTCGATA AAGCGTACCGGACATCTGCTGCTTCAAACAAAATGCCTTTCATCCACATCACAAAGTTGCCTTCGTCAT TTCTTTCTCTTTTTCTCTGTTCACAGGTGATGAATATTTCATCAATTATTCCTTGCCCTTATTCCTGAGCC TCTCAATAAAACATAAACCTCTTCGTGAGTCCAGGTTTTTATAACCAGCCTTATTAAAGATGTTCAGGG AAGTCTATGTTTTATGTTGTCTCATTAGGTTCTTGATATCCATAGGTTTTATTGTCCATAGGGTTTTGAC AGGGAAGATGGAGGAACATGAATTCTGATTTATGTCAATTAAATATTTAACAGCGATTTAACTGACAT GGTTCACCATCGATCCTTTTGTAATCGACACTATATCCTTTGTAGCATCAACTTCAATCACTTTACTTTG GCTGTTTTCTCATAACATACCCACCATATGCATCTAACATTTCAGTGCGAGACAGTCGTTATCATAGCG TATTGGCTGGCCCTTCGTACTGCCCTTGATACCGCATTGCCAAATTTAACAACAAAATGCAAAGTTTGC TCGTACAGTCAAACACGGTGTGATGTAGCATGGCGCCCGACGATGTAGTTCCGCCGTGGAGACCAATC CGATGCCGATAGATGCCGGGCATAAGCCGCACGCTTCAGCACCAGCGTCGCAGGCCGCGCTTTCTCCT CGCTCAGGTGTTCCGTTCTCGCCTCATTTATTTCGACAGCGTAGTCAGTTGTCGCAAAGTCACCTGTAG ATGACCGTCGTCCCGCAGGGAGAGCAAAACAATGTCCTCGAGTCACGCGCCCTGCCTCGGGAAGCGA CAGCAATGGGGGATGTCGGCGGGCAAGCCATCTGGAGTCGCAATACTTGTGAGGGGGACACAGGCTA GCAGCAGGGGGCATCAGCTTGCAGCCCAGCCTGCAGGGGCGGCCCTCGCCCTCGCCTCGATCTCGACC ACCATTTGTGACCTGAGCATGAACTTCCCGGATGATGCACATGTTATACCTCCCCGCCCCGCTCAAATT ACTATATTCCAAAAAAAAAAAATAAAAATTTGGTCTAGAAAGTCAGCAAGACGGGACGCAGCTCAGA GGCATGGGAGGCAACGCGAGCCAACCGGTGGATGGCTCAGTAGGGCGGCGGGCGCGAGCCCCTCGAA GCTTCAGTTGGAAGAGTGGCAACTCCGTTGCGAAAAGTTGACTGCCTTTCTTCTTCTCATCGGAGTCAT AAAAATATTTTTGTTCTTCTTTTTCGATGTTCAAAAATGGACATCGTTTGCAAAAGTGGGGTCAGCCTA ACAGATGATCATGGACACTACTCCTGTTCTCACTTTCACTAGATTGGGAAGTTATTTTCTTATTGAACT AGGAAAGTTTATTCTGATCCTGATAGAAAGTGGCCATTTTTATTCTGATGAACTGATAGGAAGCTTATT AAATATTTTTTAGTTAGTTTTTGATTATGTGGATGATAGATAGGGTTTTATTAAATATTTTTTTCTTCTTT CTGATCCAGGTTGTATTTTGGATGAACTGTATTGAACCCTCTCTGATGGTGTCACCAAATATTTTGCAG ATGAATTTAGGACAAGTTATGCTGTTTGGGTCCATAGTCAGTTTATGATTGTTATTATCCTTAGACTGA TCTGAACTCAGGGATCCTTGCCCATCAACCACATGTATTCATTTCGCCAAGATATGAGATAGTCGTCGA GGCAGAGCGGTCTCTGCCTGCCCTTGTTGAATACCGCATTGGGCGCATAGTCAAGCATGGTGACAGAT GCCGGTGGAGTGAAGCTCATTTTGCGTGGTTCTCGTTGCGGGAGATACCACGCGGCCGGGTGCCGCAC TGTTTTCTTGCTCCGCAGGTGGTTCGACCAGCGTCGGGCGGCCGCCGTCCTCAGCTGGTCGCTGATCTC GCCCTTTCAGGGCGCCGTCTCCGGGGTAGATCTCGGAGAGTTCGCTGTAGTTGCCGCGTAGTTTCATCT GTATCCTCGTCGTCCGCATGAGTCATGCTCACTCGAAGTTGCACGCGGGTGCTGCCGGCCTTTGAGCCG CACATGACCGAGGGGACATAGTCACCTCGTCGGCCTGCGCTGGGGCGGCTCGCTCTTTCGATTTGATG AACTTCTGATGATGGCCATACCTTCGGTCTGCTCACCCGCCTATATTCCAAAAAAAGGGCGCTTGGGAT TCAGGTCACGTCTGACGATGATTTAGAACAACGGGAGAGCAGGTTATCTTCTCGAGTTTTCTCAGTTGT TCGGCGGTAAATCTGTTGGTGAAAAGACTTTGTGTCATGCTTTTGATTATCTTCATTTTTCTTTCTTCAT CGGAGTCGCCTTTTATTCTTCTCGCCGATTTGTCTAAAAGCAGTACCGACGATCTAGTTCTCGGTTTCAT 
AAAATTCAAGACCATCTCACGTCTGCGCTTCTTTCTTTTTCAATCCGTGATTATGATCATTCATTCCTAG TTCCTGTTCTATTTTCTTCGTCAAATATTAAAGTTTTTATAAAAATATTTTTATTCTTCCTGATGAAACC ATGTGTTTTTGCAATATTTTTTATCTTTCCTTTCTTCCTTAGATGAGTTCATAGTGGCCCACAAACATTTT TGCCCTTTTGATGACTGATAGTGCCTTACAAAATATTTATTCTTCTTTTTCTTTCTTGATGAGTCTTCTGA TAGGGTTTTATAGGGTTTTATTCTGATCTCATAGGGGTTTATTAAAATATATTCTTTTCTGATGAGCTTA TGTAACATATGGATTTCTTTCATTAGTAAAGTTTTATTAAAATATTTTATTCTTCTTTTGATGTTCATAA AGTCTTTATTTCTTGATGTCTCATAAAATATTAGTCCTTTCTGATGGAGAAACACTAAAGTTTTATTTTA TTCTGCCACTGAGTCATAAATATTTTATTTTTGATCTCATTGTGGGCTAGTCCCTTCTCAAACATTGGTA ATATTTATTTCATTCTTTTGACATTTTCTTTTCTTTCCATAGAGTTAAATATTTCTTTTGTTCTCTTGATG GTCTCATTGTTAGTCCCTTGATATTTAAGTTTATCTTCTGATCCCCACTGTAATATTTTATTTCTTCTGAT GCATCAACCATTTCAACATCCAACAGCTCCACGATCAACAGCCTCGGCTATCTATATCACTGCACGTCA ATGCCGGTTAAAACATATAACGTGATCGCATATGGCGGCGTAAATGATTCAGTAAACCACGGGGAGA CGCTCACGGCCTTTTCTTGGCGCTGTGTCAATGTTTACACAGCATCAAATTTTGTCCGGCGATACTAAA ACGAGCCGACTCGCGCCATGGAAAACAGCCCAGACTGGTCCGTCGATGGTTCATGCAAATATTGAAAA GTTTCTTCTTCGATATTCCCCGTCAGAGGCGAACCTTCTTTTCGATGTCGTAAAGCCTATTTTTCGATGG TCTCGCCAGTCCTTTTTCGGGATAAGTCTCGCAAGATGGTTCACGTTGAGGCCAACCTCTCGGATAAAA GCATTCTTCGATGTGAGCCCTCACGTAAATATTGAAATTTTCGATGGAGTCGTAAAGCTTTATCTTCTC GAGGAAGTTCTCTCTTCAACCTTTCGATGGAGTCGTTTTATTCTTTTCGATGGTTAAATTCTGATGTCGT GCTGATGTCTCGTCAAAAATATTAAACTCTCGATGGAGTCGTAAAGTTTTATCTCTTTCTTTCGATAAA AATATACATCCCTTTCGAGTGTTTTATCTCTTCTTTCGATGGTCTCGGTCAAAATATAACTCTTTCGAGT CGTAATATTCTCTCTCGATGGTCTCATATTAACCTTCTTTTCGATGTCGTGCTTATCTTCTTTCTTCATTT GTCCCCTCACATATCCTGTTGATAGTCAGTTTTATTTTTTGATATTAAATAGTCCTGAGTCAGCTTTATC CACAGCTGAACTTTGATGATTGTCTTTGTCTCACAGTTAGTCCTGAGTCAGGACCATTAAATCCTTTCTT TGATGTCAAAGTGATCTTTTTCTGATATCCAACCTTCTGAGTCGTGTTTTATCTCTTTTTCTTTCTGTCTC ATTAGTCCTTGATGAAAGTCAGATCTTTTCTGATTGAATCTCATTGATCAAAATGACCTCACACAGCAT CCCTTTCGAAACCATCAGGGTCGTGTTTTATTTTCTCCTGATTGTCCAAAATAGTTAGTGATCTTGATGT TCATTGTATAGCTGATGGTCTCACCTCTGATGTTTTATCTTTTTTTTCTTCTCACAGTCCTTTTTCTGATG AGTCAGCTTATCTTTCTCATCAGGAAGACAGTCCTCTTTTCTGATGTTCCACAGAACAGTCCTGATGTG GGCTTTTTCTGCCTGATCCCTGATGGAGCTTATTCTCAAAGTCTCAACCTCTCATCAGCCAACTCTTGTC AGCCTATGCCCTTTTCCTCTCGATGTCTCACCTCTTTTCTGATGGTCGTTTTATCTTCTCTCACTGATGTC AAGCCTGATCTTCATTAAAATAACCTTCTTTTTCGATGGAGTCGTGCCGTCTTCTTTCTTTCGATGGTTC AATAAAATAGATGGAAATGTGCCATCTTCTTTCGATGAAACTTCATTGTACATAGTCCTTTTTCGATCT TATATGATGATATTTTTTTAGACACTAACAAATATTTAACATAATATTAATATACTAAAATTTATGTAT TATTAATTTATTTAACTAAAGTTATAAATTATATACATACTCTTTATAATTAATTTGACTTTACTATTTA TTTAGTGTCTAGAAAAAATGTGCGGACCAACGACCGTAGGAAACTCTAGAGGGTAAGAAAAATCAAT CGCTTTATAAGACCATCAGAAAGAGGTTTTAATATTTTGTGAGACCATCGAAGGAGAAAGAGATAAAA CTTTTTACGACTCCACTCAGAAAGAGGTTTAATATTTTGTGAGACCATCGAAGGAGAAAGAGATAAAA CTTTTTACGACTCCATCAGAAAGAGGTTTATTGTTTTCAGAGACCATCGAAGGAGAAAGAGATAAAAC TTTTTACGACTCCATCAGAAAGAGGTTTAATATTTTTGTGAGAAAGGAGAAAGAGATAAAACTTTTTT ACGACTCCACTGGGGAAAGAGGTTTCTAATATTTGTGAGACCATCGAAGGAGCAAAGAGATAAACCTT TTTTATGACTCCATCAGAAAGAGGTTTAGCATTTCAGAGACCACTGAAGGAGAAAGAGGATAAAACTT TTACGACTCCATCAGAAAGAGGTTTAATATTTTTGTGAGACCATCATTGAGAAAGAGATAAAACTTTTT ACGACTCCATCAGAAAGAGGTTTTAATATTTTTAGAGACTACTCTAGCTGAGAAAAGAGATAAAACTT TTTGACTCCACCCACCAGAAAGAGGTTACTATTTTGTGAGACTACTCGAAGGAGACTCCATCAGAAAA GAGGTTGCAATTATTTTGAGGAAAGGAGAAAGATAAAACTTTTTTACGACTCCATCAGAAAGAGGCTT GAATATTTTTGTGGAGAGTGAGAAAGAGATAAACTTTTTACGACTCCATCAGAGAGGCTTCTAATATT TTTGTGAGACCATCGAAGGAGAGAAAGATAAAACTTTTTACGAATTCCATCAGAAAGAGGCTTAATTA TTTTTGTGAGACCATCTAGAAGGAGAAAGAGATAAACCTTACACTCCATCAGAAAGAGGAATAATATT TTCAGGAGACCATCATCGAAGAGAGAAAGAGATAAAACTTTTAATGACTCCACTCAGAAAGAGGTTT AATATTTTAGAGACCATCGAAGAGAGAAAGAGATAAAACTTTTACGACTCCATCAGAAAGAGGTTTG GCATTTTGTGAGACCATCGAAGAGAAAGAAAGAGATAAACTTTTTACGACTCCATCAGAAAGAGGTTT AATATTTTTTAGAGACCATCAGAAGAGAAAGAGATAAAACTTTTTACGACTCCATCAGAAAGAGGTTT ATTATTTTAGAGACTATCAAAGAGAGAAAGAGATAAACCTTTTACGACTCCATCAGAAAGAGGTTTAA TATTTTTGTGAGAGACCATCGAAGAGAGAAAGAGATAAAACTTTTTACGACTCCATCAGAAAGAGGGT TTAATATTTCAGGAGACCATCGAAGAGAGAAAGAGATAAAACTTTTTACGACTCCATCAGAAAGAGGT TTAATATTTTTGTGAGACCATCGAAGAGAGAAAGAGATAAAACTTTTTTACTGACTCCATCAGAAAGA 
GGTCAATATTTTTGTGAGACCATCGAAGAGAGAAAAGAGATAAAACTTTTTTTACGACTCCCATCAGA AAGAGGTTTAATATTTTTGTGAGACCATCGAAGAGAGAAAGAGATAAAACTTTTACGACTCCATCAGA AAGAGGTTTAATATTTTGTGAGACCATCGAAGAGAGAAAGAGATAAAACTTTTACGACTCCATCAGAA AGAGGTTTAATATTTCTGTGGAGACCATCGAAGAGAGAAAGAGATAAACTTTTTTACGACTCCACCCA TCAGAAAGAGGCGTTTAATATTTTGTGAGACCATCGAAGAGAGAAAGAGATAAAACTTTTTACGACTC CATCAGAAAGAGGTTTAATATTTTGTGAGACCATCGAAGAGAGAAAGAGATAAACTTTTTACGACTCC ATCAGAAAGAGGTTTAATATTTTGTGAGACCATCGAAGAGAGAAAGAGATAAACTTTTTACGACTCCA TCAGAAAGAGGTTTAATATTTTGTGAAAGACCATCGAAGAGAGAAAGAGATAAAACTTTTTACGACCC ATCAGAAAGAGGTTTAATACTTTTGTGAGACCATCGAAGAGAGAGAAAGAGATAAACTTTTACGACTC ATCAGAAAGAGGCTTAATATTTTTGTGGGAGACCATCGAAGAGAGAAAGAGATAAAACTTTTACGACT CCATCAGAAAGAGGTTTAATATTGCAGGGAGACCATCGAAGAGAGAAAGAGATAAAACTTTTTACGA CTCCATCAGAAAGAGGTTTAATATTTTTGTGAGACCATCGAAGAGAGAAGAGATAAACTTTTAATGAC TCCATCAGAAAGAGGTTTAATATTTTGTGAATTATCGAAGAGAGAAAGAGATAAAACTTTTTACGACT CCATCAGAAAGAGGTTTAATATTTTGTGAGACCATCGGAAGAGAGAAAGAGATAAACTTTTTACGACT CCATCAGAAAGAGGTTTAATATTTTGTGAGACCATCGAAGAGAGAAAGATAAAACTTTTTACGACTCC ATCAGAAAGAGGTTTAATATTTCGTGAGACCATCGAAGAGAGAAAGAGAAAGAGATAGTTAGTTCAG ATATTTTCTTGTACAAAAGTCAATAATGTTTTAAAAATATAAGGGACAAGAATTTGTCTGTATAAAAA CTTGTGTGAAATTTTGTACCAAAAGAAAAATGTGAGGTATCCCTCATATGGATTTTACAGATCATTTAT ATAACAAAAATATTATACGATCTACGTTTTATTATATGATTTTAACGTAAATTATAAACATTATTTTGG CGACATACAATTAATTGTAAACCTAGATAAGCATAGGGGATGTTGATAAGCTCGACGAGTATATGCTG TTGGACGTTGTTTAAGAAATAGTTGATGCATCAGAAAGAGAATAAAAATATTTTAGTGAGACCAATTG AAGAGAGAAAGAGATAAAACTTTTTACGACTCCATCAGAAAGAGGGGTTTAATATTTTGTGAGACCAT CGAAGAGAGAGAAAGAGAATAAAATATTTTATGACTCCATTGAAGAGAGAAAGAGAAAATGAGAAT GAGAATAAAAATATTTTGAAGGACACCACTGTGAAAGAGGTTTAATATTTTTGTGAGCAGACCATCAG AAGAGAAAGAGAATAAAAAATATTTATGACTCCAATAGTTAGAGAAAAGAGAAAATGAGAATGAGA ATAAAAATATTTTAGTGACATTCATCAGAAAGAGGTTTAATATTTTTATGAGACCACTAAAGAGAGAA AGAGAATAAAAATATTTTGTAAACTTTTATGAGACCATCAAAGAGAGAAAGAGAATAAAAATATTTTG TAAAACTTTTTTGTGAGACCATCGAAGAGAGAAAGAGAATAAAAAATATTTTTGTAAACTTTTTATGA GGACCATCAAAGAGAGAAAGAGAATAAAATATTTTGTAAAACTTTTATGAGACCATCAAAGAGAGAA AGAGAATAAAATATTTTGTAAACTTTTTTTATGAGACCATCAAAGAGAGAAAGAGAATAAAAATATTT TGTAAACTTTTTATGAGACCATCAAAGAGAGAAAGAGAATAAAAATATTTTGTAAACTTCTTTTTTATG AGACCATCAAAGAGAGAAAGAGAATAAAAATATTTTTGTAAAACTTTTATGAGACCATCAAAGAGAG AAAGAGAATAAAATATTTTTGTAAAACTTTTTATGAGACCATCAGAAAGAGGTTTAATATTTTTGTGAT AATTCAGGCGAAATAGAATAGGAATAGGGACAGTGTCATAAATCATCACACTATTGCTGAGACAGAA AAGAAGAAGTGCAACGAGAGGCATTTTTGTGAATGTAGTTAAGAACATTTTGTTTTTGCAAACCGGAA ATATAGTGTCCGGTACACTTTTAGACCATCGAAGAGAGAAAGAGAATAAAATGGCGACTCCATTGAA GAGAGTAGAGAAAATGAGAATGAGAATAAAAATATTTGGCCTTTTCGCAACGGGTTTGCCGCCAGAA CACAGCTGAAGCTTCGAGGCTCGCATCTCTCTTCACGCGCCCGCCCTACCTGAGGCCGCCATCCACGCC GGCTGAGCTGCTGCTCCTGCCGCCTCCCGCCTGTGGTGCCTCCTGAACTGCGCGTCCGCCGTCTAGCTC CCGGGCAACGGTACCGGACTCGCAGAAGCTTCTAAAATTGAAATTTTTATTTTTTTTTTTTGGAATATA AATGCAGGAGCAAGGGCGAGAGGATAACATGGCCATCATCAAGGAGTTCAGCGCGCTTCAAGGTCCA CATGGAGGGGCTCCGTGAACGGCCAATGAGTTCGAGATCGAGGGCGAGAGGGCGAGGGCCGCCCCTA CGAGGGGCACCCAGACCCCTGCCAAGCTGAAGGTGACCAAGCGGGGTGGCCCCTGCTCTTCGCCTGGG GACATCCTGTCCCTCAGTTCATGTACGCTCCAAGGCTCACGTGAAGCACCCCAAGGCCGACATCTCCG GACTACTTGAAGCTGTCCTTCCCCGAGGGCTTCAAGTGGGAGCGCGTGATGAACTTCGAGGACGGCGG CGTGAGACCGTGACCCAGGACTCCTCCTGCAGGATTTTAAGGAGTTCATCTGGCTAAGGTGAAGCTGC GCGGCACCAACTTCCCCTCCCGACGGCCCCGTAATGCAGAAGAAGACCATGGGCTGGGAGGCCTCCTC CGGAGCGGATGTACTTCGAGGACGGCGCCCCTGAAGGGCGAGATCAAGCAGAGGCTGAAGCCGGAAG GATATGGCCACTACGACGCTGGAGAGGTCAAGACCACCTACAAGGCTGGGCAGTCTCCGTGCAGCTGC CCGGCGCTCAACGTCAACATCAAGTTGGACATCACCTCCCCACAACGAGGACTGGCTACCATCGTGGA ACAGTACAACGCGCCGAGGGCCGCCACCCACCGGCGGCATGGGATGAGCTGTGCCAGTCTAGATTCG CCACCATGTTTGCATTTTGTTATTCGTTGCCCAATGCGGGTGATGTAATAAAGGCAGAGTATACGAGA AGGATTATGCTCATATATTTATCTTTTTTGACTATCCTCACTTTGAAGCTATCTTACAGAGAGTGCGTTA AGATGCATGGATAGATATGTTGAATATAGATAAACTGGTAGGAAAACCCGTAAAAGTTAAAGTGATT AGAGTTGATTATACAAAAGGATATATAGATGTCAATTACAAAGGATGTAGACATCAATAATAGCGGCC GCCC TTACTGAA 
TAAAGTGATTTTTTCACTGGGTAACATTTTTGAGTGATTAATCAGATCAGAAAGAGGGTTTTAATATTT CAGGGAGATCCATCGAATAGAGAAAGAGAATAAAAAATATTTTTGTAAACTTTTTATATATCCACTAA AGAGAGAAAAGAGAATAAAATATTTTTGTAAACTTTTTATGTGAGACCATCAAGAGAGAAAGAGAAT AGCAATATTTTTGTAACTTTTAGAGACCATCAAAGAGAGGAAAGAGAATAAAAATATTTTGTAAACTT TTATGAGACCATCGAAGAGAGAAAGAGAATTGTAACATTTTAGAACTTTTTATGAGACTGACCAAAGA GAAAGAGAATAAAATATTTTTAGAACTTTTTATGAGATCCCGACCGAAGAGAGAAAGAGAATAAAAA TATTTCCCAACTTTTTTATGTGAGACCATCAGAAAGAGTTTTAAATATTTTGTGATACTCCTGGAAAGG AAATAGGAATAGGAATAGGAATAGTGTCATAATCGTATCACACTATTGAGACAGAAAAAGAAGAAGT AACGAGAGGTAACTTTTGTGAATGTAGTTAAGAACATTTTTGTTTTGCAAACCGGAACATAGTGTCCG GTACACTTTTAGACCATCGAAGAGAGAAAGAGAATAAAAATATTTTATGACTCATTGAAGAGAGAGA GAAATGAGAATGAGAATAAAATATTTTAGTCTTTTTTCGCAACGGGTTTGCTGCCAGAACACAGCTGA AGCTTCGAGGCTCGCATCCTCCTTCACGCGCGCCCGCCGCCCTACCCGAGGCCGCCATCCACGCCGGTT GAGTCGCGTTCTGCCGCCTCCCGCCTGTGGTGCTCCTGAACTGTGTCCGCCGTCTAGCCTGGGCAACGG TACGATCCCTGCGCAGAAGCTTCAAAAATTGAAATTTTTTATTTTTTTTTTGGAATATAAATGGTGAGC AAGGGCGAGGAGGATAACATGGCCATCATCAAGGAGTTCATGCGCTTCAAGGTCCACATGGAGGGCT CCGAACGGCCACGAGTTCGAGATCGGAGGGCGAGGGCGAGGGCCGCCCCTACGGAGGGGCACCCAGA CGGGCCAAGCTGAAGGTGACTGGGCGGCGGCCCCCTGCTCCTTCGCTCCGGGGCCATCCTGTCCCCTC AGTTCATGCAGTACGGCTCCAAGGCTCACGTGACCCCGCCGACATCCCTCCTCTCGACTACTTGAAGCC GTCCTTCCCCGAGGGCTTCACAAGTGGGAGGCAAAATGAACTTCCTGAGGACGGCGGGCGTGGTGACC GTGACCCAGGACTCCTCCCCTGCAGGACGGCGAGTTCATTCATAAGGTGAAAGCTGCGCGGCACCAAC TTCCCTCCCGACGGCCCCGTAATGCAGAAGAAGACCATGGGCTGGGAGGCCTCCTCCGAGCGGATGCA CCCCGAGGACGGCGCCCTGAAGGGCGAGAGATCAAGCAGAGGCTGAGCTGAAGGACGGCGGCCAAT ACGACGCTGAGGCCAAGACCACCTACAAGGCCATAGCCCGCAGCTGCCCGGCGCCTACAACGTCAAC ATCAAGTTGACATCACCTCCCACAACGATGACCACAGCCTTCACGTGGAACAGTACGAACGCGCCGAG GGCCGCCGCCACAATCTACCCGGCGGCATGGACGAGCTGTACAAGCTCCATCTGCCACCATGCTTGCA TTTTGTTACCTGTTGCCCAATGCGGGTGATGCAATAAGGCAGAGTATACGAGAAGGATTATGCTCATA TATTTTATCTTTTGACTATCCTCACTTTGAAGCTATCTTGGCAGAGAGTGTTAAGATGCAGGCGGATAT GTTGAATATAGGGATAAACCAGGAAAACTGTAAAAGTTAAAGTGGATAATTATAATAAAGGATATAT ATAGATGTCAATTCACAAAGGATGTAGACATCAATAGGCGGCCGCCCAAACTTGTTTATTGCAGCTTA TAATATTACAAATAAAGAATAGTGATCACAAATTTCATTGAATAAAGCATTTTTCATCAGCAAATATTT TAGTGACACCATCAGAAAGAGGTTTAATATTTTTAGGAGACCGATCGAAGAGAGAGAAAGAGAGAAT AAAAATATTTTGTAAAACTTTTTATGAGATCCATCAAAGAGAGAAAGAGAATAAAATATTTTAGAACT TATGAGACCATCAAGAGAGAAAGAGAATAAGTAAATATTTGTAACCTTTTTAGATGAGACCACTAAAG AGAGAAAAGAGAATTGAAAATATTTTTGTAAACTTTTTTTATGAGACCATCAAAGAGAGAAAGAGAA CAAAAATATTTTTGTAAAACTTTATGAGACCATCAAAGAGAGAAAAGAGAATAAAATATTTTTGTAAA ACTTTTTTTATGAGACCATCAAAGAGAGAAAGAGAATAAAAATATTTTTGTAAACTATGAGAGACCAT CAGAAAGAGGTTTATTATTTTGTGATAGAAAGGAAATAGAATAGAGGGAGTGTCATAATCGTATCACA CTATTGACAGAAAAGAAGAAGTAACGAGAGGTAACTTGTGAAGTTACAACATTTTTGTTTGCAAACCG AATATAGTATGTCCGGTAATACACCATCGAAGAGAGAGAAAGAGAATAACAAAAATATCGACGAAGA GAGAAAGAGAAAATGAGAATGAGAATAAAAATATTTTAGTTCTTTTCGCAACGGGTTTCGGCGCGAA ACACGCTGAGGGGGCTGCAGAGATTCTCTCTTCACGGCGCCCGCCATCACCTGGAGGCCGGTCGTACT CCTCCCGCCCAGGGTGTTCTGTTTTTCTGAATCAGGCCAGTCCGCCCAGCCGCCCGGGCGGTACCGGAT CCCTGGTAGGTTTTTAAAAATTGAAATTTGTTTTTGAACAGTAAATGGTGAGGCGGGCGAGGGACATG GCCATCATCATGAGTCTGCTGCGTGCGCTTCACATGATCGTGACCCAGGCCACGAGATCGAGGAGAGG GCAGAATTCCTGCGCACCCGACCGCCGTTGAAGGTGACCACCAAGGTGCCTCTGCCTGGGGACATCCT GTCCTCGCCTGGCGTACGGCCCACGGTCACGTGAAGCAATCTCCCGCCGACATCCTCCTTCGAAGGAC TGCTCACGTTTCGTCCTTCGAGGGCGAGCGCGCGCGTATGAACTTTCAGGCGTGAAACCTCCCGCGAG TTCACTACAGCCGCCAGCGGCACCAACTCCGCAGAAGGGAGTGCGATACCGTGCGAAGGACCATATG CTGGCGCCTACAACCGTCAATAACATCAAGTTGGACATCGCCTCCCACAACGAGGACTACACCATCGT GGAACAGTAATGAACGCCATTGAGGGCCGCCACTCCACCGGCGGCATGGACGAGCTGTACAAGTCTA GATCTGCCACCATGTTTGTGCATTTTGTTGTCTAAGGTTCACTGTGGCAGATGTGAATAGCAGAGTATA CGAGGAAGGATTATGCTCTATATTTATCTTTTTGACTATCCTCACTTTGAAGCTATCTTGGCAGAGAGG CAGATGTATATGGATAGATATGCTGAATATAGGGATAAACTGGTAGGAAAACTGTAAAAGCAGTGAT TGAGTTGATTTATAAAAGGATTGGTATAGATAAAGTCACTCATAAAAGGGATGTAGATCAATAATAGG GTGGTCAGCCTAAACTTGTTTATTTGTAGCTTATATTTGTTACAAATAAAGCTAAATAGTGACTGCAAT TTCACAAATAAAGTATTTTTTCACTGCAAAAAATATTTTAGTGACAATTCCATCAGAAAGAGGTTTAAT 
ATTTTTAGACCACTGAAGAGAGAGAGAATAAAAAAATATTTTGTAAAACTTTTTATGAGACCATCAAA GAGAGAAAGAGAATAAAATATTTCTGTAAAACTTTTTATGAGACCATCAAAGAAAGAGAAAGAGAAT AAAAATTATTTTGTAAACTTTTTATGAGACCCATCAAAGAGAGAAAAGAGAATAAAAATATTTTTGTA AAACTTTTATGAGACCATCAAAGAGAGAAAGAGAATAAAAATATTTTTGTAAACTTTTATGAGACCAT CAAAGAGAGAAAGAGAATAAAAATATTTTTGCAAACTTTTTTGTGGTGAGACCATCAAAGAGAGAAA GAGAATAAAAATATTTTGTAAACTTTTATGAGACCATCAGAAAGAGGTTTAATATTTTGTGATACCCTG AAAGGAAATAGGAATAGAAGAGGAATAGTGTCATAATCGTATCACACTATTGAGACAGAAAAAGAAG AAGTAACGAGAGGTAACTTCTTTTTGTGAATGTAGTTAAGAACATTTTATTTTGCAAACCGGAATATAG TGTCCGGCAATAATTTTTAATTGTGGTGTCGAATCGTTCGATTAACCCTACCCATCCAATTTCAGATGA ATAGAAGCTATCGATTCAGCATCATCGCTTTGAGTTTTGTTGAATCGATGAGTGAAGTATCATCGGTTG CACAGATGCCGATCATACTTGAATCCATCCTTGACCTCAAGTTCAGATTTTCGATTCCTTGCACATGTT CCGATACGAACGCTAAACTCTAGATTCTTTTATCATCATTATTTTGTATCGACGATCGTTGGAACCGAC GATATCTTCGTAACTCACTTCTCTTATATTAGACCGGAGTACTGATGGGTCTTGATGTCGCTGTCTTCTC TTCGCTATGATCTGATGTCGATAGACACCCTCACAGTCTTGATCATAAAGCCAGAGCTTCTTCACGAGT GGATCGCGGAGAGTCCTTACCTGTCCTGTACGCTGGACAATCTAGCATTCACTGTGTTTCCATCGAAGC GGATTCTGAGGATGTGATTTAATCTGAGGACGGTAATCCAAAGTTCATTCAGGACCTCCAATTGATGA TGGAGTACAAGTGGTAGGAGGATCACATCTCGACTGGATGTGGAATCACTTCTGATTCCAATCTCGAT CTGGATCTGACTCGGACTCTGTAATTTGCTACGGATTAAATCTTATCATTGGTCGGTGTTTGGTCTTGCT TTGGTGGACTTTGATAATAACATCGATTCCTATAAGGATGTTTGTTTTCTTCTTCAAACACGAGGAGGA GGATGAGGATTGCTGAAGACTGGCAGCAATGATGCATGCCAGGACGATATATTGTTTCATAATTCGTT ATTGATTGAGTACTGTTCTTTATGATTCTACTTTTACTCATGCACAATTAGAATATATTTTCTAATTTTA CGAGAAATTAATTTATTGTATTTATTATTTATGGGTGAAACTTAGCCTATAAAAGCGGTGGTTTGGAAT TAGTGATCAGTTTATGTATATCGCAACTACCGGGCATAGTATTATCGACATCGAGAACATTGTACCTAC ATGATAAAGAGATTGTATCAGTTTCGTAGTCTTGAGTATTGGTATTACTATATAGTATATAGATGTCGA TAGATATATAGTCTCCGGAATGCGGCATGATACCGTCATCATTCTTTGCTTCGCTAACTGTTTGGAGGA AGAATCTTTGTTGCATTTAACTCGAAATTCAGAGTGCACACCTTTCTCTCCTGTAAAGAATCCTGAAGT CGCTACCTTATTAAGAACGAGAAGTATCCATCACGAAATACGGGATTATGCCTTATGGATTCATAGTA ATAGTTAGTTCCGACGTTGAGATGGATTCAATTGAGACCGGTAGTGGTCGTCCGAGGCTAATGACGTC GTTGACGGGACATGATTAATTTCCACATCGATATAGTTAAAGGTATTTCTGGGTATGGGTTCGCATTTA TCTGCGGAAGAGACGGTGTGAGAATATGTTCCGAGACCACACGGAGAACAGATGACGTCTCCGGATA CTCCGCATCCTATTCCACATTTTGTTTGGGAAACACATGCCTTGCATCCGGATGAGATCCTTTTGAGAA CATTGGGCCAATATCTGGGAGAGCAGACCAGATTCTATTGTGAGTGTTACACGATCGTCTTCCGTTACA ACTTAGACAAGCGGGTAAATGATTATTGGTGAGATGTGAAGTACTCCGAACCACACGGCGTACATTGT GTGTTAGCGTCTTGCTAATGCGCATAATCTGAAGCGCGTATGTTCCCGGACACAAATTATGGCGTTTGT ATTCGCTTGGTTTCTTTACACTTTCCATCGGATGGCATGCGGTGCTATATCTCTTCCGTTTATTATTAAT ATACATGAGAGAAACAATATATACGAGTATAATACGGACTTCATAATTTAATAATGTAGTAATCAAGT TGTCGTTCCTGTTTCCTACTTCTCCAATCATATAGATATTTTCTTTCTATCATGGATAATATTTGTAATG GTTCTTCCGGTACAACATACTGTTTAGATGATATTGCGCATAATTTCCGAGGCAAATACGATAGTTCAG ATTGACCGATGGTAGACTCCTAATTTATTGAGTGCTTTGTCGACGAGTTTCATTTCTTACGCTCCATCG ATAGATGGCACTGTTCTATGAGATCGTCGTACATGGGAAATGAAATGTGTTCGTCCGAATGTATGATT CCAAGATAGCTGTGATACCGTATACAGGTCGGTATGCTGAAAGATTCGAATCTCTTTGAGGCGACTTA TGTCACGATGATGGAATCTATCTTACTGAATGATATATTTTCATATAAATACACTTTTATAGTCCGTTTA AACAGAATTTACTATGTAGTTCCGCGAATGACTCGTCCCTTAATAGGCAGTAGGCTAGTATCTTTTACG TAGTAATCGTCGTAGGGAGAGAATTCTGACATCTTGTAGAACAACGATTTAATCATAGGTAGAGATAC TTTCAGTCTGTGGTGGATGATAAACATTCACAACATCCGCCTTGTATATGATGTTTCTGTTTTCAAACA CCAAGTCGAATACCGTCTTAGTCGGAAGGTTGATGTCGTATCCGATGTATGAGGCAAACATTGAGTTG TTACACTTGAAAATATGGCATATAGTATTCGTCTTTCTGAATGTCGAACCTATCTAGTAGATACCGTAG TATATTGAGAGTGTATCCTTGATTATGTTTTTATGAATAGATAAAGTAGATGGCTGTCCTTCTTCCTTTT GTTCGCCAATTCGAGTAACATTGGCGAGAATATGACCTGTTGCACAATCGTTCCATGATGGGTGTACA ATCAAGATTATTACGTATCCTCGAGATAAAAGAGCATACACCACACGAGACTATGTTTGGTATACTGT TGAAGGTAAGTGTGTAACCGCGTTAATGTTTGCTCCATAACGGCTATCGCGTAGATGAATTGCCGGCT CGCATCTTAGTGACTTAACTTGTAATAATTGCTTTGGTAGAACGTGGATATGTGTTTACAGTAGCAATG AAGAGAAGAAAGCGTTCATCCTCGTCGGCGCAATTAGGCTTCCATCCTTTGTACAGAACGCAGTAGTT TAAGCCCTACTGAATTTATATCTAAGATAACACAGCAATAGATCGGATGATTTTACTAAAGTCATCAA TGGTGTCCGAAAGTTAGTATATCAAAGATCTTGTTATCGATTGATAGTGAATGAATCAGATAGTGGTG TCCTCCTTTTCATCCTTGCTATCAAAGCATGCAGTGCCGCAGGTAACAACAATATCTTAATACAGATGG 
ATTTCAACCGTGTATTCATCGTATAGCAATGTAATGGAGAGTTACCCTGTTTATTCAGATCGCAAGTGT TTAATAACTAGCTTAACAGATGAGACGATGTATCCACATCAAAGAACGTAAAAATACATATGACAAAC ATTGCTGACAGAAACGTGACCTTCATTCTTACCTCGTCGTCCATAAATACGTTAGGTATGTACCATATA CTGTCGCGAACGATGCTCACAATCTCGTCCATCTCGCCCATCTCTTATTATTTCCATCATTTACTTTTTT CATAATTAGAGATGTACGAAAGAAAAAAGAAAAAGAAAAGAAAACAGAACAATATATTTTTTAGTAA TAATGTTTATGCGAGACATATAAAAATAAACTCCGTGTTTATGATAAAGCTGGTAAATGTTTTATCCAT CTTTGGACGGAATCAGATTTTGTAATATGTCATGGAAACAAATGAAACAGGACATTATCAATTCCATG ATAAACAATTATTTAATGGAGTAATAGCATTCCATGGGTAATTTCGAAATCAAGTTCGTCTGTATTAAT GTTGTCCACTATGGAGTCGATCCTCCTAATTGTTCTTACAGTTTCTGCAATGACGTTAGTTTTTTTGTAC CATTTGATGTCGGATTCTTTGCGTATTCTAGTCTGTGGCGTTTGCTTCTGTTTAAATAATATATCAAACA TGGAGACGCCTGATATGTAGGCATTCTTCATTCTATTAATGTTCACTTATAGCGCTTTAGTTCCTTATGA TTAATCGGTGATATCAATACTTACTTTAGAAGGAAAATCATCATCTAGATTAAGGCGCATCTGATACA GGCGAATAATGTCCAGGATATAGATATGCATATCTCATTAAATGCGTCAATCATAGTCTCAGAGGGAT GGCAGCTAAGTAATAAATCAAACTTATCCCTCGTTTTGTTTTCTTCTTGGTAACTGCTTTTCTCTGGATG GCCGTATTGATTATCGAGCGTGATGTTGTAACACTCTGCCCATATTCCAATAACCGCTTTGCAAATTGT ATATTATTGACATCGACCGTGTAATATAGTAGAGTTTTATTCTCATTATCGATCATATCTATATCATCCA TGTACTTGCTTTAGTATATCAAATACATCTATTAGTATGGTTTCATAACAGTGATACCCGCAATTATTA AATCTCGATATCAGACCGTACATACATAGACGGGTCATTGTTAGATATGTGATTTAAGGCAGCCGCGT GTCCATATTTTCCACGATAAACCCATGACGTTTTAGCATCGGACGAGACATATTAACAAAGTAGCGTC GTGCAGAGGGATAGTTGTTGTCCGTTATCTAACATGCACGAAACTAATTCATATCGCCGTAATGTAAG TAGTTTAACATCAACATGGCTTGGTACGATGGATTCATCCTGTTCAACCCTTTAGAATGTTATCGATGA TGTAGTGGTTATATTCTTGAATCGTACGGAAGTAATACTACGCATTACGTCGACAAGAGCATGACGTC TCTCAACAAGAAGATTAACGATTTGGCGTTCACATTATATGGGGTTACTGCAACGTTTAAGCTTAGATA ATACGCCTCTTAATATAGGCTGACGTCGTATACCTTACACGTGTCCACATCCTTTATTAATAATAATTT AACAATCTCTATATCTATGGTTGAGAAAGACCAGTAGTATTGGATGGGTAAAGATCCTCCTTCGTCTCG CCATATGGATGGAAACATTGCGCTATGGATCAAACATTTAATTACATCCCTGGATAGAGATTGAGATT CTCTATGAGACGATATATAGTAATGAAGAGAGTTCTTACAATATTATCACTGTTGTATATACAGGTACG AAAGATGCAACCGGTGCTGTAAACATCTCTGATTTAATAGCCATAAGTTAGTTTCTGGTCTCGGATTAG GCGTCGTTACGTATATATCCACCCAATCCGATGAATTCATTGATTGTATAATTGTACTTGGACGGACGT ATCCGTTTATCCACAATTAGGTATTTGAAGACGTAAGTCGATTATCCGAAGACAGATCGAAATCATTT ATATTCGACTTGAGTTCGTTAGAGGAATTCGAATAGCTGGATATCGCAGGATGCACAAATCTGAGATT TTGTATTTACTTCCATGTTTACTGTATGCTCCTAGCGGAGTTAACCTTCGTTGTTTCTACAAAGTCTCGA CTCCGCGAGAGAGTAACAGTCGAACAATCTTAATGTCTGTTATCGCATTTATTGGAGACGTAACAATA AAGTGCATTGTTTCTCGTTCATCTATATATGTTTTGATAAGTTGTGACACGTTTCAATCTCTAGTTTATT TTTTTTGTACGTCACATCTTCATCCAGTAGACGACATAGAATAGTGTACCTTCTACCACAATAATCCAT AGCTATTCTTGGTGCTAATATTCCTATTTCACGAAAAATGATGAAGGCAATCTGGCCTCATAAGATGAT GATAAAAAAGTGTAGTGAGAGAGCATGAAGGAGATTTAGTATTTAGCAGTGCGGATGTTGATCCAAG AGGGTGAGATAGTCGTTCTCGTCTGTGAATCTTTCTCGCAGCATAAGTAGTATGCTCGATATATTTATC GTTGGAAGACTCTTCCAGAGACGATAGCTGATTGAGTACAAAGTCCAATGATTGCATCGAAGTTCTCG GCGGTTTTCAGTGGAGTCATTTCTGATGAAACATTTAATGATCTCACTTGCCTGTCGATATTGTCCCAC GGAAGTGAACTCTTCCAACTCACCACCAAAGAGCTCCGTTGCATCAGTTCTGAAAGAGATGAGAAGCC TGTAGAGAGACCCTGCGCTTTCTCTATGGGTCCATCTATGAGAAACCCACAGGATGTATTCAGTCAGA CAATGTTCTCCGACGTCGGCCACGGTATTCAGGGAGTCCCTTAGTATGTGGCAATGACAGGGTCTGAA TGTTACCAAGGAAAGGCCATTGTAAAGGTAGACCTAGCCGTTTATGCTAATAGAGGCTTTAATTTCATT TTTAGAATGGGTTGTGGATGAGGAATGAGAGTGATATCATATTGAGAGCGTAGTTATGTAGAGGTGTA TTTCCTATATTATTTACTTTCGGTTTATTTTACCAACTCTTTTAATAAATTTCTTTTCACGATGCATCTTA GTTGAATGATCGCGTTTTTCATAAGTGGACATATAGATGCAGAAGTAATGAAGAAAAAGTATTACTCT ATCATCTACATAATTAGGGTCTGCTCCTTTTTAACAACTTATAGCATGTAGTAGTAGTTTACTAGTTCA AATCAAGTCTAGAATATATAGTGGATTAATATATTTTTATATTAGCTAAAAAGCTTTATCTATACTCAT CAGAAAGCATATCATTCTCAACTTCATCATGAGTTAAATATTTGTGTAATGGATGTCATGAACATTAAA CGTATTCATGACATACCTTTAATAGGTTTTTAAACAGTGATGATTCAAATCCTTCCATTCATTAGATAA CAGTGTAACGGAGTCAAATTCTCTTACTAGTTTGTTTATATCATAAGCATCTCTACAAACAGTCTAAAA CAACAATAGAGAAGACGGACAGATTCTTTTAACGCATAAATGACACATGTTATCAAATGATATTCGCT GGATGAATTATTATTAAACGTAGTTATGATAAATGATCTCTAACGACATCTCTCGCTAGAGATAAAAT CTAGTATCAAGAACATTAAACATCTTTGCATCATACTCGCATAGCATAGTTTTTCATAATTATCACAAT ATTTAAAAAGACTTATTCGGAAAAGTATTTTACATGTATCATCGATGGAGATCCATATGAGGAGTCAC 
TAGTTCTTTCAGTAGTAATAACAGTGCTATCATTCGATAGTATAATTATATAAGGCGTAGAAGGTTCAT ATGTTGTTGTAATTGAGTAACCGTTGGTAGTTCTGTGGAATCAATAATTATACTAACAGCAAATAGTAT ATAATATATAAATATGTTCCGTTGATATCATTATTTTAATGAACCCATTTCTAACACCCTGTTATATCGT CCAATTAAATGTAGCCAAACAATCTACCATGTTCTCGATTGACTACTTGTACGGTAGCGACGCTGACTA TCTTTTATTGTCTCTGCTCCAATTGAATGTCGCGATACAACGCAGTTTCTTCTTAAGTGCATGTTTCATA TCACCACGAACATAAGTCGGCAAGATATATAGCCAGAGATACTTGTAATCATGATTGCTTGGTCATAA ACAAGCCCGTCAATAATTGTATACATATTCAGTTTAGAGCAAAATAATTAAGCACAATAGCGCTTAAT CTCAAAATATGTTATGTTTATTTTTTCATATTAAACATACTGGTTAAAATCCTCTAAAGGCTGATCTTCT ATCTATAAATCAAGACTACCATTTAGACAGTGGTTTCATGTTTATAAAATGTTCTTTTGTGTGAATAAG GAATATGTTTAATCAATAATCAACCATCGACCCCATTACGATAGTATGCAGGTTAAACCCCATTAGAG AGGTACGTGTAATCAGTCTCTCCAGTTTTAGTATTTTTATAAGTCATTACTACATAAACGGCTTTTAAA CAGTCTCCTCGATAATAAGCCATATCTGAAATTCTATTAAATACTCGAGTCATTTTACGCACGGTCAAA AAAGTAAGTAATGCCGACGACTTCTACATCTCTATAGAACACCTTAGAATACTCATTTTCTTTGGAAAA TATCCTCAGACTCTGATTTAACAATGCACGACCTATAGTAAAACCGCAGACCAAGTTATATTAGTCAA TGGTATATCCAACCATCAGGTGTGGATAGTCCAGTCTTTGGTATCGATAGTGTAGTTATTGAACTGAGA AGTTACCGTATAAATTTTTGGTCATCTCTAAACAAGGAAACTAATAATTCTACACTATTGAACGATTTA TCTTCCGTAATGGGTGGACTAGCGATGATGAAGTCACGAATATAAGACACGCTATTAATCCGTATATC ATCATTTTGATATTACTTATAATAACGATTTGTTTAATTTTAGTTTATACTATTAATTGTAAATGATATT ATTATTTTTTAAGTATTATCAGTTTAGTTTATACTATTACTATTTCTGTAATATTTAGACATAGATAAAC GTGATAAAGTCTATTTGTTTATATTTATTGCGGATAGCAGTATTTCCATAAACGCAGCAAGGAGATGTC CTAGGGTTTGACTATACATGTGAATGATTGACGAGGTAGATCTGTAGCCGATAGGTTCATCGAAAAGA CTGTAATACTCCTGTAACGGGCTGATGCTTCCTAGGCACTTTCATTAATGACGAGAACGTCGGGAATTT TTCATAAAGATAGTTTCTTATTACATCTCATAAATATCCGGAATTATTGCAGTCCCGAATTTCTTTAAA ATCGAGAAATTCAGATTATACGGTTCTATTATTTCCAGACTTTTAAACTTTGTATTATATTAAGTCTATA CTTTTGGTAGATAACATACCATTAATCATTTTAATTTTTCATCCATGCTTTTATGTTATACACCTCTATC GTCCAGTATAATCACCATTACTAGAGTTTCTCGTATGGAATATCGATAATAGAGACCCTCCGAATAATT CATTAGCGGCTATTGCAAGTTATTCTCGGTACCACCATCATATCAACACTGCACCATATTCTTAGAAAT ATAAAACTTGTCTGTATATGTAAGATGTGTTTAGAAAAGCCGGATATTTCACATTGCTTTGTAAAATGG ACGGCGCTAACAACTGTCATATTTATATTAATGGATAGCGGATTAGTCAATAAGGAATTACTCTTACC ATTTGTCACGTCTTAACCCCATTCGCTGATTAGTTCCTTTGTTTGGTTAGCATTATTAAAGCTTACAGCT TGAAAATCGTCTTTATTTTTGTAGGAAGGAGGCGTGGAATCCCGATACTATCGCTACCGTATATTTTAT TTGCGGTAGCGCTAGTGTCTGCACAATACGAATATCTACGTCCATGTCATTATTGCTCATCGGGTGTAT TCTCATTCATATTCTCTATATATTTTGATAGTTGTTCAGCTGTAGAACCAGCTGCCCATGATTTAGAACA GACAGTAGATAAAATAGAAAACCGAGAAACAAAACATTTTCATCAGGGTGTTTTACGATTAGTTCTTT AAAGATATCCATGGTATGTGGACCAAACAATAACGATAACGATATATATCATAAATAGCCAATGTTAA ATTTCAGTTTATGTTTGGTACCCCGTATTCATACATTTCAATAAATTGGTATTGCGTACACAATCAATC ATATTACATACCATTAATAATGCAAGCATAAAAATCGTTAGTAGATGTTTCAACATAGGTTCCGTAAG CAAAGAATATAAGAATGAAGCGGTAATGATAAAATCAATCGTTATCTAAAATGATCATACTCATTTAT TTTATTCTATTATATTAACACATACATTTTTAAATAACAGCAACACATTCAATATTGTATTGTTATTTTA TATATTATTTACACAATTAACAATATATTACTAGTTTATATTACTGAATTAATAATATAAAATTCCCAT CTTGTTATAAACACACACTGGAGAACAAAGCATAAACACAGAATCCATCAAAAATGTCGATGAAATA TTCGATGTTGTTGTTCGCCGTTATGATAATCAGATCATTCGCCGATAGTGGTAACGCTATCGAAACGAC ATTGTTGCAAAATTGAAAACGCTACAAACAGATATTCCAGCTATCAGATTATGCGGTCCAGAGGGAGA TGGATATTGTTTACACGGTGACTGTATCCACTAGAGATATCGACGGTATGTATTGTAGATGCTCTCATG GTTATACAGGCATTAGATGTCAGCATGTAGTATTAGTAGACTATCAACGTTCAGAAACCTAAACACTA CAACGTCATATATCCCATCTCCCATTATGCTTCATTAGGCATTATTATTGAAGCGGCGCACGAAAACGC GAAAGCGTTTCACGATAAATGCGAAACTAGGGAAACACGATAGAACTGGAAAGGCTTCACGTAACTG AACGAAGTACAACAG

$\underline{\text { Isolate } 5}$

ACGGTACTTCGTTCAGTTACGGCGTATTGCTCTTGTCCAGGGTTTGTGGCCTTGTTTCCGCATTTATCGT GAAACGCTTTCGCGTTTTTCGTGCGCCACTCACCCAAGATGTTCAATACTTCATCTGAAGCAAAAGATC TTGTGTCTAAACAATGAATACGCTTTATACGATTTCATCTTGAATAGATCCATAAACGGGGGCTCCATG AATATCGTGTTTGAAGTAACGTCGTCGGATACATAAGAATACTTTGTTCAATTACGGCTTTTGGATTTG CTCCAATATCATCCATTCTTCATCACTCCGTCGAAATCAGCATTTTTGAGAGTTGGCAATTCGGGAGAT ATTTTGATAGTATCTCCTTCGGTAGCTCCTGATAGATGAAACGATGACGTTTAACCATCTATGTAGAGA CGGCTGTCTTCCAAAATAATGCCTTATATTCTTGAACGGCTTCTTCACTGCCCAATCACCAGGCAATAA 
ATGTATTTTATTTTAATAAACTTTCCTTGGCATATTCTTGTTAATTGGTTTAATCGTTTATTAAGTAGAA TTTAGCAGTTTGAGACGCTAATAGTTGTTTAACTTATCCTGTAAAGGCATTAACAAATATCTTTTCTGT AGTGTATTTCCTGTATATGCGGGCATTCCTACCTCGTAACGGTGATAGATGTACTGGGACCAATTACAG ATCTAGCGGTCTGATCTTTTCGTCGAGGGCGACAATATAACTTCTAATCTTATTATTTTTGCGGATGTA ATATATGATAAATTGATACTGGAAGTGTTGTAGAAATAATTTTAATATCATCGTATTCTGTACTGCCTT CTGGATAACCTGTTCATCCAGCATTCAAGTTACAATTCTTAACGATCATACCTAATAAAGTAAGTTAAT TCATTGGTCTCTTTGGGTACATACTATCTATCAAAAACTAATAGCCGGTCTAATAATCAGCGGAGGGAT GGAAAGTAGTCGCAACATAAAATAAGTTGGCTGGATATTGATGGGTATAATAATGGCTAAAACTTTTT CATGAATAGAAATTAACTTTTTGATAGATGAAGGAAAGTGGAACGTTAATATCATCCAACTTGTTGAC GAAACAAACCTTTCTTGAAAAGTAATTTTTGATACGGTTGCATACATTCGCTGTTCCAACATGAGCTTT TCTTGGATAATATTTATCCTTTAATGCTCCCAGAGCGTGTCCCATTCAACTCTTTAGGTTAATATCGTCC AGACCGGTTCTCGTGAACACCAATAATCCGCAGTGAATACATATATGATTCGGTAAACGAATAATTTC TGAAATAAATTCAGGCTTAACTATGAGGTTTTATAAATACTTACTTACCCCAGTGACCAAGAAACATTC AATTCCGTTTCCACAAGTCTTGTAATGCCCCATCCATAGCACCTAGTCTACGCTCTTTAACGGTACCGA TATCGTCGTCATTTTTTAACACTGACTAATGATAATATCTGTATTAATCTCTTTTGATCATATAGACTAT ATACGTAACTAGAGATTACAACCATTTTTATCAAGTCAGTTTCTTTTAAAGAACCAAAGTATACAATCA AATTTCCCTTTTTATTACAACTATAAAATAATAGTTATATTTACACACTTTAAATTTTTTATCATGACAA GGACGAACAAATTTATGCATTCTGTGATGCTAACAAAGACGATATACGATGTAAATGTATTTATCCTG ACCAAAAGCATAGTACGGATAGGAATAGATACAAGATACCCTATTATTATTGTTGGTACGAATAACCA TGTAAACGAAGCGATGCGTTGTTACCAGCCTCTTTAAAAAATATAACAAAATGCAATGTGTATCGGAT TGTACCATTTCATTGGGAAACGTTTCCATTACAGATAGTAAAATTAGATATGTAAATAATGTTTGTGAT TCCCAAACGGTAGCTACCGAGAATATAGCTGTCCGCTATTTAAATCAGGAAATTAGATACCCTAATTA TGATTGATGGCTTCCGATTGGATTACTAGTTAGCTATTTTAATATTACTTTTCTAAGCAAGATATAGAA TTATAAGATACCAGATATATATTGTGATTGTATAATGTTCTTATCTCATCTCTACTAATTGATTAATCAG CGACTGAAATAGCAGATCTATCGGCTATCTCTACTCCAGTTACCATGTTGTACTTGGAAAAATCTAACA ATTTTTAATGGTATATTAGGGCGATAGAATCTTGACAACTATTTCCGTCTCCCAACATTTTGGGAAACG AAAGTCTTTTTTACCATCGCCTGTTTGTAGTACACTATTAACTATATTAGTTTCTGTAAGTATCTAGTCT AACAAGCTGAAATAGAAGTCGAGATATTACCACATCTATGTATAGAAAATTTACAGAAACTTGTTATG ACCTTTTTGAAGATACGATGTTATAATGATTGGTTCTCCATAGTCATCATCAACCCGGGTATAATTTAT GGATGATTCGTTAAATACCTGAAACACAGTTTCCATAGAATCAATTTTATGTCTAACAGTTGTCAAAAA GTAACTAAATCATTATACTCTATATCGGTAGTATATCTCAGTAGTACGTTTCTATTTATTACGCGTACG GATCTCTTGCTATTTCTGTTTTAGACATAGAATTTTGCTAGATATTTTTACGTTGTATTGGTTCTTGACT AACTTTTCAGTTGATGTTGTTGAATATTTAAGAAACGAAATATAGGTGTTGTAGAAATAGTACCTTTGC TTTAGTAGTAGGAAATGTTTTATTGCAGTACACGGTCCTCAGCATAAAGTACATGTAGAAAATAGTCA TATTCTGATTAGGATAATCAAAGTTAACAACTACTTTGTTACGGACGATCTTATTAAGGTAGTACATCT TTTCATAATTTACAGCGTCTGATTTGGTAACTCGAGGTCCAGTCTCATGTTCTCTTTCGGTATAAATACT TAATAATCTCATTCAGCTGAATATGAAGGAGCAAGGTTGTAACATTTTATTACAGTGTGGGATATAAA GTCCTTGATCCAGTGATCTGGAAACGGGCATCTCCATTTGGAGCTAGATGCCACGGGTTTAAAATACT AATCATGACATTTTGTAGAGCGTAATTGCTTAGTAAATCCGCTTAACACTAGGTTCATTTCCTCCTCGT TTGGATCTTATCAGAAATTAAATAATCTTAGAAGGATGCAGTTGTTTTGATGGATCGTAGATATTCCTC ATCAACGAACCGAGTCACTAGAGTCACATCACGCAATCCATTTAGGAATAGGATCATGATGGCGTCCG TCAATTAGCATCCATTTGATAATCATTTCTAAATTGCCAAAATGATCTCTCAAATAACGTATATGTGTA CCAGGAGCCGATCTATGCATACACTACGGTGGCACCATCTAATATACCGTGTCGCTGTAACTTACTGA AAAAAATAATTCTCCCTGAGCCAATAGTTTTAACGCTGTCCTTGATACGGCAGTTTTGCGACCTCATTT GCACTTTCTGGTTCGTAATCTAACTCATTATCGAATTTCACTCAAAATACATAAACGGTTTATCTAACG ACACAACATCCATTTTTAAGTATTATATTCTAAAAATTTAATCAATGTTTATTTTTAGTTTTTGAATAAA AATATAATATTATGAGTCGATGTAACACTTTCTACACACCGATTGATACATATCATTACCTCTATTATT TCTATCTCGGTTTCCTCACCCAATGTTTAGAAAAAGGCCTCCTTAAAGCATTTCATACACACAGCAGTT AGTTTTACCACCATTTCAGATAATGGAATAAGATTCAAAATATTATTAAACGGTTTACGTTGAAATGTC CCCATCGAGTGCGGCTACTAACTATTTTCCTTCGTTTGCTGCCATACGCTCACAGAATTCAACAATGTC TGGAAGAACTGTCCTTCATCGATACCTATCACGGAGAAATCTGTAATTGATTCCAGGGCATCCACATA GTTTAGTTGCTTCCAATGCTTCAAAAATTATTCTTATCATGCGTCCATAGTCCGTTCCATTATCTATTAT CGTTAGAATATTTATAGTCACGCATTTATATTGAGCTATTTGATAACGTCTAACTCGTCTATTAATTCTG TACTTTTACCTGAAAACATGGGGCCGATTATCAACTTCAGATAATGTCCGCCGTTCATGATGACAATAA AGAATTAATTATTGTTCACTTTATTCGGCATTTAATATATCCATCACGTTAGAAAATGCGATATCATGA CGAGGATCTATGTATCTAATGGGATCTATTGCGGTGGTAGCTAGGGGATTCTTTTTGAATCGCATCAAA 
CTAATCACAAGTCGAACAAATATCCTTTATTAAGTTGACCCTTCCATCTGTAACAATAGGGACCAGCGT TAACTGGTTTTAAATCTTGAGAGTCTGTGAATTTTGTGGTGTCTGTATTCCTCTGAAGAGATTCATAAC AATGACCCACGGCTCTAATTTGTTTTGATTGGATCAATAATAGTGTAGAAAGTCTAGATATTGAGTGAT TGCAATATATCAGATAATGAGAGATTCATCATCTTGACTAGCCAAATAGCAAATGAATCATCCTCTGC GAGAACATCGTTAAGGATACTGGTTGTGATCCATTTATTGATCATAAAAGCTTTGCACAATCTTTATAC TATCGGTTTACTATTTATTGATAACGCAGATGTTTGAGTTGTCATCCATGGTAATCCATAGATCATTAA TTTATCGTCTTCACGCTAGATTAGCACGTCGTAATCTGTCAATAGAGGATCGAGGTATTTTTCGTTTTG ACGCGAAGAACATATTTAATTCAAGATCTAAAAATACATATATTAGGAATGAATACAACAGGAATACT TCAAATAAAACTATTAATCTGTGTTTATAAACGCAATAAAGGAATGTTTAAACGTGGGCTCTATAAAC TTTTAAGAGTTAAAGTATACATTAGGAACATTCTCCATTTATAGTAATCAATCCTTTGTCCGGAATATC TGTTAGAGAATATTCTTTTAACACATTCCAATAGTCCCAGAAAATCATTTAACCTAATAGGTTCTTGAA GAGGCTAGAACTATACGATTCTAGTTCCTTATTAGTACTAAGTTTCTCTAGTTTATTTTTATTGGTGAAT CCGTAAATGGCATTCAATCTCATGGATGTGGAAGGAGGAATACACATTCCAAGGACATTATGAATTCC GCATATTTGGAATATCAAAGCCTTGTTGTCTCCGATAGGTGTATACTCAGTCGATGCGGATTCCATATT TTTCTTTATAAATATTAATCTTTTACGAGTTTCGAAAATGCACAAGATGAGTGATGATCCTTGACGAAA TAACCATGTTCTTATTCTTTCTCCCTACCACTTTTTAGGAATGACGGCAGTCATATCGTATTATTAATAA TAAGGGGCATAGCTTCATTGTTAGGATCCAAAATGGTTTTCAATGGCTTCAGCAGATTTGGTTTATCAT CTCCCTAGACCCAAATTGTTTTCTTATATTATACTGTTGTAGTAAACATTGCTCCTGTGATATTTGCCTG GAGTATGAATCAACTCCAACTCATATTTTGACATCGTTCTAGGTACTATATTAAAATAGAAAGCATAG ATCTTGGAAATTTGGATTTTGCGCCGGCAATAACCATTGTAAATCATCATACTCAGATATGCTTCCTGC AAAAATATGGTATCTTCTTCGATGAGGTTTTCTAGCAGGCGCTCATTTAGAAGTTTTTGTGATAAATGA ATACCCATACCGATGTTTACAAACGATAATATAGACAAAAATCCAACTAAACGGTGATAGAAATATAC CAGGAAGAAATGAAAAGATTGTGGCCAAAATAGATTCAACAACGATAGACCAAGGCCTAAACCAAAG ACTACAGCCTAATCAGCCACCGAAACAAGATAATAAATGCAGAAGTGGAGATTTTATCAATATTAGTT GTGTGCCTACGAAAAGGAATATTGCAATGACGGATATCTATCTCCTGCCTATTATATGTTAAAACAGG TGGATGATGAAGAAATGAGTTGCTGGTCAGAACTATCGTCGTTGTGAGATCCAGAAGGCGGTAGGATT TCCTCTATTAAAAGGCGGCTAAACGTATTTCTGTGGATCGATGCTATATTTTAAACAGTTTAAAAAACA GTAAAGTTGTGAAATTAACCCACTGGGATTAAATGTTTAAATGATACTGTTATTTTCAAACTGTAGTTA TTTTATATTCAATGTATAAACGTGGCATATATTCTAACAATTTGTTTTGATCTGGTTTCTATTCCCAGAA CAGACATTATTTTTTCTGTTAATCAATTAATGTTTAACATTTGTGCAGACATATTGGTAGTTCTATCTAT TTACGGCAACCGGCTCTATAGAACAATCTACCACAGTCGTGTTACTTAAATTTCATGCAGCCATGAGA CAATAGCCGTGAGGATATGTTACTCAATTACTTTTTCGGTGGTTGATAAAAAATCACATATCGCTATTG ACCAAGCAGAACGATGGATATTCTCAGAGTAAAGAAAAGAAGTATGCTACAGGAGCACCAGTAAATG AGTTGTTGAACCTGGTACACTGGTATATGTGCCCAAGAAGATTATTACTTTATGAGCATGTCACTCACC GATGTGTCAATTAGCAATAATGTCAGGTATTATTTTCCACAGATGGAATAGTGTTAGAAATAGAAGAC TTTAATATCAAGCATTTGTTATGGCAGGTGAATGTTTGTTAGAAGTCAGTCTAGTACTATTATGATATA AAGTAATAAAAAATAGTTAATGTGATGACTAGCGCCACCAACGCCAACAACATTTGATAATTTCTACC TAGACGTACCGTAAAAATATATAAATTACTATAACAAATAATGATATATCAATAAACAACCTAATTAA TGGTCGAGTATAGCGAGGACATTGATGCTCTAGACCGTGTATAACAAAATCTACAAATTTTTCGTCCG CTATATTTTGTTTCACTATATCGTCGATGATCAGCGACCAACTTCCATGTTAATCTATTAAAATAACCA TCAGCGTATATTTTTTCGTTTGCATATCCGTGGTAGCAATAACCATCGGAGAAGTTCTAAAGAATGTGT CATGTAAGTAGTCAATGGACGTTTCCTTATTGGCTAAAATAAGTTTGATTTTATCATTGGTGGATGTGA ACAGCATACGCTTGTAGATACATAAGCACTGCCAATATTATAACACCGATAACAATCATATAAAAACT GAACTCCTGTACCAGCAACTTGTCTAGGTGCTATTTAGGTAGTGGCCTTGGTAGTCCAATTGCATCAAC GCCTTAATGGCACAATTTCCTTTGCTAGATCCTGTTAATAAGTTCCAAATTTGTTGGAGATCCTGGGGC TCCTTAACATTCATCTATGATTACGTTTGTATCTTTAATTTGTTATCGACGACCATACTTCAAATTTACA GGTTGTTTTACATAATTTTCAAAATCTCTGGCAACAGTGTTTACACTCGTCTGAATGTTTAACTTGCAG CAGTAAACATAGCTGGCGTATGCTTTGTTCCGGTGTTAATCCACTACTATGTTTCTGTAGCGGCTGATA ACCACAGCATCAACTGAGCATCCGCGTCCTGAAACACATATTTTTAACTGTGAGTTACATCCATGGTTT TGGCCGGATATAAAATTTCCGATTTCTATATCACATTTGTTTTGAACATAGCATTCACTTCTTGTTCTAA TTTAGACGAGATACGTTCAGCTAGGTGTATTCACCGTCGTCTGGCAATAGCGCTGCGGCTTTTCCATTT TAAATAGCTACAATTAGTATCCATGTGCAAGAGATAATAAACTGATCAAATGCAATTTTAGGTCGAAC GAGTGTTTAATATTATGTTGAACTACTTTTTGCTCTATTGACGGGAACAGTAGAAAATCTATCACTATT GCTTAATCCACATGACAATCTTAATGAGAGTTTATCCATCTGTAAGTTGTTCACGCTAGTATTACATCG TACAATATTGCAAAGTCTAAATTATTATAATTACGTGTTAGCAAGAAATTAACATTGGCATTCGAACCT CTGGATCCCAACATTCTCGAGGTTCCGCATTTTAATGACTCTTCTAACTTATCTCTAGTGGGATAACTA 
CATCTCCTTATATTTCTGTTTAAAGTCCGCAATTTATTTGTCTTAGAATATAATCGATCATCTCTTTGCT ATCTTCTGTATTGTGTGCGTAAATGATGCAGAAATGATTCACATATTGGTACACTAGCATCTTTACTAC ATAATGTTTGCATCTTATTATACAGATTTATTAACGATTGTTGACCCTCTACAGTTCTATTACTCTATTA AAATGAACCGATCCACTGATGGCATATGTTTCTATCGAACGTATCCCCTGACACCAGTCGAATAGAGA AATCAGCATCGCATTTCCAGTGTCGTGAACGTCTGGCCAACGTTCTAATACTGCACCCTCTTCATACTT GTCTGATATCTTTCCATCCGCTTTTTCCAATAATGAGTACGATTAAAGTGGAGCAGCAGTGGGTGCGGT TCCACGACAACCGCGATTGTCTTAACAATGCCGAAAGACCACCGGGTCCTGTTGACTAATCTAAACTC ACGGATATCTTTTTCTTTACTAGCTTTTCACCAGCAGGTTTGCCGCAGAACGCAACTGGAAGCTTCGAG GGGCTCGCATCTCTCCTTCACGCGCCCGCCGCCCTACCTGAGGCCGCCATCCGCGCCGGTTGAGTCGCG TTCTGCCGCCTCCGCCTGTGGTGCCTCTGAACTACGTCCGCCGTCGCTCCTGGGCAGCAGTGGATCCCT GCGGAAGCTTCTAAAAATTGAAATTTTATTTTTTTTTGGAATATAAATAGTGAGCAAGAACGAGGAGG ACAACATAGCCATCATCAAAGGAGTTCAGCCTGCGCTTCAAAAGGTCACATGGAGGGCTCCGTGAACA GCCACGAGTTCGGATCGAGGGCGGGGCGAGGCCCGCCCCCTACGAAGGCACCAGACCGCCGGCTGAA AGGTGACCAAGGGTGGCCTGCCCTTCGCCTGGAACATCCCCTCAGTTCATGTATACGGCTCAAAGCTA CGTGAAGCACCCGCCGACATCCCCGACCGCGGGGCGCAATGTCCTTCCCAGGGAACTTCAGAAGTGGG AGACGCGTGATGAACTTCGAGGACGGCGGCGTGGTGACCGTGACAGGACTCCTCCCGGGACGGCAGT TCATCTGAGTGAAGCTGCGCGGCACCCAACTTCCCCTCCGACGGCCCCGTAATGCGAAAGACCATGGG CTGGAGGCCTCCTCCGAGCGGATGTACGGAGACGGCGCCCTGAGGGCGAGATCAAAGCAGAGGCTGA AGCTGAAGGACGGCGGCCGCACTACGGCCGCTGAGGTCAAGACCACCTACAAGGCCAGAAGCCCGTG CAGCTGCCACGCGCCTACAACGTCAACATCAAGTTGGACATCACCTCCCACAACGAGGACCTGCCACC ATCGTGGAACAGTACGAAGACGCGCCGAGGGGCCGCCACTCCACCGGCAGCATGGACGAGCTGGCCT GGAAGTCTAGATCTGCCACCATGCTTGTTTTGTTATTCGTTGCCCAATGCGGGTGATGTAATAAAGAGG GCAGTATACGAAGGATTATGCTCTATATATTTATCTTTGACTATCCTCACTTTATGAAACTATCTTGGC AGAGAGTGTTGAATGCATATGGATAGATATGTTGAATATGAGGGATAAACTGGTAGGGAAAACTGTA AAAAGTTAAAAGTGATTAGAGTTGATTATACAAAAGGATATATAGATGATCAATTACAAGGATGTGG AGCATCAATAATAGGCGACCGC

TTTCACAAATAAAGCATTTTTCACTTTTGCAAAAATAAATTCCAAATTTGTTGGAGATC TGGGGCTCCGTAAACATTCATCTATGATTACGTTTTGTATCTTTTAATTTGTTATCGACTGACCACTTGC TAAATTACAAGTTGTTTTACATAATTTTCAAAATCTCTAACAACAGTGTTACTCGTCTGAATGTTTAAC GCAGCCAGTAAACATAGCTGGCACGTGTCTTTTGTTCAGTTAATCACTATATGTTTCTGTAGCGGCCTG ATAACGGCATCCAACTGAGCATCCGCGTCCGCAGAGCACATATTTTTTAACAGTGAGGTTACATCCAT GGTTTGTCGGATATAAAAATTTCCGATTTCTATATCACATTTTGTTTGACACTAGCATTCTTCTTGTTCT AATTTAGACGAGATACGTTCGCTGAGTGTATTCACCGTCGTCTGTATGCTTGCTGCGGCACCATTTAAA TAGCTACAATTATCCATATTACCAAGAGGATAATAAACTGATCAAATGCAATTTAGGTCAGTGAGTGT TTAATATTATGTTGAACTACTTTTGCTCTATTGACGGGAACAGTAGAAGAATCTATCACTATTGCTTAA TCCGCGACAATCTTAATGAGGAAGTTTTATCCATCTGTAAGTTGTTCGCGCTAGTATTACATCGTACAA TATTACCCAAGTCTAAATTATTATAATTACGTGTTAGCAAAATTAACATTGGCATTCGAACACTCTGGA TCCCAGCATTCTCGAGGTTCCATATTTAATAGCTCTTCTAACTTATCTCTATTAAGGATAACCCATCTCA TATATTTCTGTTTAAAGTCCGCAGACTGTTGTCTTAGAATATAATCGATCATCTCTTTGCTATCTTCTTA TTGTGTGCGCGTAAATGATGCGAAATGATTCACATATTGGTACACTAGCATCTTTACTTACATAATGTT TGCATCTTATTATACAGATTTATTAACGATTGTTGACCCTCTACAGTTCTATTACTCTATTAAAGGCTGA ACCGATCCGCTGATGGCATGTTTCTATCGAACGTATCCCCTGACGCAGTCGAATAAATCAACATCGCA TTTTCCAGTGTCGTGAACGTCTGGCCAACACGATTCTAATGCTGCACCTCTTCTATTACTTATCACTGAT ATCTTTCCATCCTTTTCCAATAATGAGTACGACAAAGTGCATTGTAGTGGGTCGCAGTTCCATTATGCG ATTGTGTAACAATGCCGAAAGAACCACCGGGTCCTGTGTTGACTAATCTAAACTCTGGATATCTTTTTC TTTACCTAGCTTTTTTCGCTTGAGTTTGCCGCCAGAACACAGCTGAAGCTTCGAGGAGACTCGCATCTC TCCTTCACGCGCCCGCCGCCCTACCTGAGACCGCCATCCACGCCGGTTGAGTCGCGTTCTGCCGCCTCC CGCCTGTGGTGCCTCCTGAACTGCGTCCGCCGTCTAGCTCCTGGGCAACGGTGCGGATCCCTGCAGAA GCTAAAAATTGAAATTTTATTTTTTTTTTTGGAATATAAATGGTGAGCAAGGGCGAGGAGGACAACAT GGCCATCATCCAAGGAGTTCATGCGCTTCAAAGGTCCACATGGAGGAGCTCCGTGAACGGCCACGAGT TCAGTCGAGGGCGAGGGCGAGGGGCCGCCCCTACGGGGAGCGAACCGCCAAGCTAGAAGGTGATAAG AGGTGGCCTGCCCTTCATACAGGACATCCTGTCCCTCAGTTCATGTACGGCTCCAAGGCCTACGTAAAC GCACCCGCCGACATCTGACTGCAAGCTGTCCTTCCCCGAGGGAGCTTCAGAAGTGGGAGCGCGTGATG AACTTCGAGGACGGCGGCGTGGTGACCGTGACCCAGGACTCCTCCCTGCGGGACGGCGAGTTCATCTG AGTGAAGCTGCGCGGCGCAACTTCCCCTCCGACGGCCCCAGTAATGCAGAAGAAGACCACATGGGGC TGGGAGGCCTCCTCGAGCGGATGTACCCCGAGGACGGCGCCCCTGAAGGGCGAGATCAAGCAGAGGC 
TGAAGCTGAAGGACGGCGGCCACTTTCCGACGCTGAGGTCAAGACCACCTGAGGGACAAGAAGCCCG TGCAGCTGCCCACGGCGCCTACAATCAACATCAGGTTGGACATCACCTCCCACAACGAGGACTACACC AATCGTGGAACAGTACGAACGCGCGGGGCCGCCACTCCACCGGCAACCAGACCGAGCTGTACAAGTC TAGATCTACCACCATGCTTACATTTTGTTATTCGTTACCCAATGCGGTGATGTAATAAAGGAGCAGGAA ATTACGAAGGATTATGCTCTATATATTTATCTTTTGACTATCCTCACTGAAGCTAATATTGGCAGAGTG TTCAAAGATGCATATGGATAGATATGTTGAATATAAGGGATAAACTAGTGAGGAAAACTGTAAAAGTT AAAGTGATTAGAGTTGATTATACAAGGATATATAGATGTCAATTACAAAGGATGTGTGAACATCAATA ATGACGGCCACCCTAAACTTAATTTATTACTTGGCTTATAATGGTTACAAATAAACAATAACATCTAAA ATTCTAAATCGTATTTTCACAATAAATTCCAAGTTGTTGGAGATCCTGGGGCTCCGTAACGTTCATCTA TGATTACGTTTTGTATCTTTAATTTGTTGTATCGACGACCGCGCTAGAGATTACAAGTTTGTTTTACAGG TACTAAAATCTCTAACAACAGTGTTTACTCGTCTGAATGTTTAACACAGCAGTAAACATAGCTGGCAC GTATGCTTTTTGTTCCGGTGTTAATCCGCTATATGTTTTCTGTAGCGGCTGATAACACAGCATCAGCGC TGAGCATCCGCGTCCGCAGGCACATATTTTAACAGTGAGGTTACATCCATGGTTTTGTCGATATAAAG AATTTCCGATTTCTATATCACATTTGTTTGACACTAACATTCGGCTTCTTGTTCTAATTTAGACGAGATA CGCGTTCGCTGGAGTGTATTCACCGTCGTCTGTGTCTTGCTGCGGCCTCCATTTAAATAGCTACAATTG GTATCCATATTGCAAGAGAGATAATAAACTGAGTAAAATGCAATTTTAGGTCGAACGAAGAGTGTTTA ATATTATGTTGAACTACTTTTGCTCTATTGACAGGAACAGTGAAAATCTATCACTATTGCTTAATCCAC ATGACAATCTTAATGAGGAAGTTTATCCATCTGTAAGTTGTTCACGCTAGTATTACATCGTACAACAGC CCGCAAGTCCTAAAATTATTATAATTACGTGTTAGCAAGAAGTGCTGTGGCATTCCAGACACTCTGGA TCCCAACATTCTCGAGGTTCCGCATATTTATTGACTCTTCTAATAACTTATCTCTAGTGGGATAACTAC ATCTATATATTTCTGTTTAAAGTCCGCAGACTGTTGTCTTAGAATATAATCAATCATCTCTTTTGCTATC TTCTGTATTGTGTGCGCGTAAATGATGCAAAAAATGATTCTATATTGGTACACTAGCATCTTTACTACA TAATGTTTGCATCTTATTATACAGATTTATTAACGATTGTTGACCTCTACAGTTCTATTACTCCCTATTA AAGGCTGAACCGATCACTGATGGCATATGTTTCTATCAGACGTATCCCCTGACACCAGTCGAATAAAT CAACATCCTCATTTTCAGTGTCTTGAACGTCTATTAACACGATTCTAATACTGCTTTCCTCTTCATACTT ATCTGATATCTTTCATCCTTTTTTCAATAATGAGTACGATTAAAGTGCGACAGCAATTGGGGTGCAGTT CCATTATACGATTGTCTTAACAATGCCGAAAGACCACCGGGTCTGTGTTGACTAATCTAAACTCTGGAT ATCTTTTCTTTACTTTATCCTCTTTATCTTTTGCTAGTATTGGTCCTATATGCACATATTCTAAAAGCTTA GCGGGGAACTATCGTCATGCATTTTATCCACGTTTAATAACATCTCATCAGTGGGGTACTCCCGAGGCG GATCCCGTTTAGGAGCTCAGCACTACTCCGCCACCTTATTTATCTCATTGAAATTATTAATCTAAAAAC GCCATAAAGATGTTGATCTTAAAGGATTGAACTCTATCCGAAAACAACATTCTAGAATGTTATCGTCA TTATCATTACGATTCTAGTTTCAAAAACGATGACTCTCTTTTGAATCCTCGTAGTTTGTTGAGACAGAA TAGCTATTTTGAAGAAAACTTTTGTAGTTCTTGAGAACATTCAGTCATAGAATATTCCTGGAAAATGCA TCAGTATTACCAGAGGTCTTCATAATAATATTGTCATCTTTAAACATAATAGCCAGATGCTGATGCTGA CTAATACATGATAAAGCCCAACAACCATTCTAAATGAATGACAGTTTCTACCACAACATTTTTCTTCAT GCGAAAATTAAACACATAAAGATTTCTTTTGATCTATACTTAGACAAATAGTAGTATCTGTCCTAATGA CATCAATTCATATTACAATCGACACTTACACATGATAACTAGAAAGTTTTACTAGGTATTTTTCAGATC AGGTTCTATATCTATGATGGCATCATTGTAGAAAACTACAAACACGATTTTACCCCTTGGACACAGGA AGATTAAACACAATGTTTCGGCTCCTCGGTAGAACACTGATGCACTTTGAGAGAGGGTATAATAGACT TTAAATGTTTACAAGATCCGCCAAGGCGGCAAAATACATTAACTCATCCGTCGAGGTCATTTATAGAC ACTTCTTCACTGAACTCTGAAAAATATGCACCTGGTAGCGCAGTTTATCGATTTTTATACGGATGCTCA TTTTAAATTTTTTGTAAATTATTTAAGTTAAATGGCTGCAGAACAGCGTCGTTCTACAATTTTGACATA GTTTCAAATGCCATAATTGCAATCTGTATTGAATATATCTATTAATTCTGAATACATAAGTCCAAAGCT AAACAATTGTGCTGTGTCGGCATCGAAAAGTCGGTGATTAATGGTATCTACAATTGTTGCGAGTCAAA TATAGAAATAATGGACAAGAGCAGCTATTAAAAATATTGGACAATCTTCGATGTCATTCGGCTCTATG TATGTAACACCACAGATTTCTGGGGCTATATAATTCGTTAAAACAGTTTACACTCATACTACCACATTC TTTATACATACAAGCCCACTATTCTAACCACGCTAAACACTTTATTAACCTGATTTTTATCTAACAAGT TGTGTATGCGGCAGAAATGGTAGAGTATCTGAAGACCAACTAGATTCATCAAATAAATCAATGTCTCA AGAGCTAGCAAGAATTGTGTGGAAATGAAATATGCTCTCATTAATCTGGTACAATATAGGATTTTGCC AATGATCATCAGTGAGCCTATTATAGTAGCTGTGGATTTCTGGTTAAAACCAACTCTGATTATTCTGCA GAAGTGGAAAGGCTAATGGAACTACCAGTTAAAACTGATATAGTGAATACCATGACTGTAGCCAGAA AGGTATTGATACTAGCAACAATATGAAACAGAATATATAAACCGGCAGAAAATAGAAGGAAATTGAA AAGGTAGAAAAATATTTACCAGAAGTTATATCTACAATTGCCAATAGTAATATAATAAAAAATAAAAA TCTATCTTTCAGGCCAATATCAACGATAAACAGATCATGGAATGCTCTGAATGCTAGACACGAGTGAG AAATACTCTGGGATACTCGAAACTGATGGAACTGTGACTAGTCCATTGACGGGAAATAATACAATTAC AACATTTATACCAATTTCTGCGTCCGATATGCAAAAGTTTACCATTTTAGAATATCTTTACATTATGAG 
bioRxiv preprint doi: https://doi.org/10.1101/2020.10.26.356402; this version posted October 27, 2020. The copyright holder for this preprint

(which was not certified by peer review) is the author/funder, who has granted bioRxiv a license to display the preprint in perpetuity. It is made available under aCC-BY-NC-ND 4.0 International license.

GAAATTGATGGCAAACAACGTTAAGAAAGAACGAGAGGAAAAAACAACGGAGGGTAGTTATGCATAT TAACTCACCCTTTAAGGTAATCAATTTGCCAAATGTTAAAATTAGAATCATATATCTTTATCCATCTAT TATCGTCTGTGAAGTTTATCAGAAAATATCTTTACCTAATATTCGTTTTAGACGATGTTTCAACACCGG CATTTTTATAACAGTCAGCTACCAATTTAAAACAATACATTCTATTATGACCAAATCCATACGGAATAC CCAATAAAATTGGCTTGTATCAGCTGCTATTTTCATAACTTGAATATTCTCTAACATGTATGCTTTTGCA CTAAGATATCCTTCAAAAACTATCCAATGGGACATTCAAACTCGCACTATGGGTAGATTCAGCTACCA AGTGTTCCGATAATAACGTACTATAATAAATACTTATGTTTTTTCTATCGATGGATTCAAATGACTTGT TAATGACATGGTACTCAATTAATAAAATAATAGAACCTCTAGGCGCATATGTCTTGATAAATTAACCG GATCCATATTAGTTTATATCCAATAGAGGTTGCACTGTTAAAGTTACGTTGAGGTTCTATTTGTGTGAA TATAATTTTAACCTACAACATTCGTTCTTTACACTGACAGTACATCCTTTAATGATTTTTCGTATAATCA ATTTATATCGTTCGTGAGAAATATCTTTACCGCATGTAGAGGCACACGGTTGAACACCATTTTAATCTG GTAACCTATATTTTATTATATTATCACTTTTAGTATTGGTTGATGACAGACTATCGTTGAAGTAAAAGA ATTATTTATACTCAGTAGTAAATTATCATATAGATGCGAATACTATCATTATTTACAGTTTTCGTTGGG CTTAATCCGAAGTCGAGGATATAGATTTTCAACATCGATATCTGATTTAGTATTTTCGAAATCTATATA AGATGATAAAGTTTCTTCTGAAACATCAATCTCCTTGCGTGGTTCTAGCGCGTACATTAATTCAACAGG GTAACGGAACGGTCTCTAAATAGATGATTTAAAAGAATTGTTCCATTTTCATCTAATGCGGAAATATA AATTCTTGAACAGTGCATTGACAAGGAGGAACATACAGTAGACAT 